\title{
The Compound I of Nitric Oxide Synthase: The Active Site Protonation State
}

Kyung-Bin Cho, Etienne Derat and Sason Shaik*

Department of Organic Chemistry and the Lise Meitner-Minerva Center for Computational Quantum Chemistry, The Hebrew University of Jerusalem, 91904 Jerusalem, Israel

Supporting Information 


\section{Methods}

Calculations have been done using the Chemshell ${ }^{\mathrm{S} 1}$ application combining Turbomol $^{\mathrm{S} 2} / \mathrm{Jaguar}^{\mathrm{S} 3}$ and $^{\mathrm{s}}$ DL-POLY. ${ }^{\text {S1 }}$

Turbomole/Jaguar:

The hybrid B3LYP ${ }^{\mathrm{S} 4}$ functional was used throughout this study. QM/MM geometry optimization was done with Turbomole using a double zeta basis set (B1), while energy evaluations were done with a Jaguar single point calculation on that geometry with B2. Several other "large" basis sets were used with Turbomole in the beginning to find a suitable large basis set (B2W being representative). Jaguar B2 was chosen for the energy evaluation due to convergence issues. Spin and charge values, in the text, are according to B2 calculations. Jaguar was used only for single point QM/MM using a patch for Chemshell written in our laboratory (by E. Derat). Polarization of the QM layer by the MM layer is taken into account by inclusion of point charges in the framework of the charge shift method. Test calculations were made with B1 on the same structures (1R and 1P) to ensure that the Jaguar and Turbomole generated energies were compatible within the Chemshell scheme (Table S0.1).

Table S0.1. Calculated absolute energies using the Chemshell scheme with different QM programs (Hartree).

\begin{tabular}{|c|c|c|c|c|}
\hline & \multicolumn{2}{|c|}{ QM energy in QM/MM } & \multicolumn{2}{c|}{ Total QM/MM energies } \\
\hline & $1 \mathbf{R}$ & $1 \mathbf{P}$ & $1 \mathbf{R}$ & $1 \mathbf{P}$ \\
\hline Jaguar $^{\mathrm{a}}$ & -2961.733215 & -2961.699805 & -3112.191964 & -3112.148257 \\
\hline Turbomole & -2961.733093 & -2961.700338 & -3112.191953 & -3112.148825 \\
\hline
\end{tabular}

${ }^{a}$ This is obtained in Jaguar after subtraction of point charge - point-charge interaction energy (marked "(D)") from the total energy.

B1: LACVP (Turbomole)

B2: LACV3P*+ (Jaguar)

B2W: Wachter all electron basis of Fe, 6-31+G* on the immediate Fe-coordinated atoms, all oxygen, all nitrogen (except on Arg 375), all $\mathrm{C}$ atoms in $\mathrm{H}_{4} \mathrm{~B}$ and the central $\mathrm{C}$ atom in the substrate and the carboxylate $\mathrm{C}$ in the heme propion arm. The rest is treated with 6-31G.

DL-POLY:

Standard CHARMM parameters were used, however, no parameters were available for $\mathrm{H}_{4} \mathrm{~B}$ and $\mathrm{SO}_{4}$. Therefore, those parameters were built from scratch (see attached files).

ORCA:

Using the ORCA program, ${ }^{\mathrm{S} 5}$ the Mössbauer parameters were obtained from single-point B3LYP calculations (at the geometries obtained after QM/MM optimization). The isomer shift was evaluated from the electron density at the iron nucleus. ${ }^{S 6}$ Iron was described by the triply polarized core properties basis set $\mathrm{CP}(\mathrm{PPP})^{\mathrm{S} 6}$ and the other atoms by the $\mathrm{SV}(\mathrm{P})$ basis set $^{\mathrm{S} 7}$ with the inner s-functions left uncontracted. For the iron atom, an enhanced integration grid was used and the overall integration accuracy was increased to $7 .{ }^{\mathrm{S} 5} \mathrm{MM}$ point charges were included in these calculations to probe the effect of the protein environment. 


\section{QM/MM preparation:}

\section{Reaction 1:}

The initial coordinates were taken from the PDB database, file id 1NOD. Only the A-subunit was used. The missing residues 101-107 were added manually using Insight II. Two waters were deleted in the active site, and $\mathrm{O}_{2}$ was manually added. The force field used for the MM part was the CHARMM force field, but the parameters and topology files for $\mathrm{H}_{4} \mathrm{~B}$ and $\mathrm{SO}_{4}$ were built manually.

Hydrogen atoms were then added to the structure, and energy minimized. An energy minimization then followed on the manually built residues 101 to 107 with the rest of the enzymes fixed.

The enzyme was then soaked in a $16 \AA$ water layer (7000 water molecules), whereby the inner $8 \AA$ layer water molecules and residues 101-107 was relaxed by a minimization-equilibration-minimization scheme. This was repeated another 4 times. In total, 7522 water molecules were added, in the last addition 61 water molecules.

During the QM/MM optimizations, the following residues around the QM region were optimized: Enzyme residues Trp84, Ser112, Ile113, Met114, Thr184, Ala187, Trp188, Ala191, Pro192, Arg193, HEM194, Ile195, Gly196, Arg197, Gln199, Leu203, Ser236, Ala237, Ile238, Gln257, Trp340, Tyr341, Pro344, Ala345, Val346, Met349, Phe363, Asn364, Gly365, Trp366, Tyr367, Met368, Glu371, Arg375, Asp376, Arg382, Met428, Phe437, Ile456, Trp457, Leu458, Tyr483, Tyr485

Cofactors $\mathrm{H}_{4} \mathrm{~B}, \mathrm{Arg}_{\text {substrate }}$ Crystal Waters 101, 103, 105, 115

Solvent waters 3322, 4270, 4349, 7121, 7184

\section{Reaction 2:}

The singlet structure from Reaction 1 was used with a proton added to $\mathrm{O}_{2}$. Appropriate changes were made to the parameter and topology files, one additional cycle of soaking and minimizationequilibration-minimization was done. Water 4278 was added to the MM optimization sphere.

\section{Reaction 3:}

The singlet structure from Reaction 2 was used with a proton added to $\mathrm{O}_{2}$. Appropriate changes were made to the parameter and topology files, one additional cycle of soaking and minimizationequilibration-minimization was done. Water 4318 and 7126 was added to the MM optimization sphere. 


\section{MD simulation}

Using the PDB-file obtained from the procedure described above, we performed an MD simulation for $300 \mathrm{ps}$, with the heme residue and outer solvent water layer (8-16 ̊) fixed. Figue S1 shows a 293 ps snapshot showing the solvent molecules forming a hydrogen bond from the heme out to the solvent. In contrast, only one of the water molecules is present in the region at $0 \mathrm{ps}$, a crystal water molecule already present in the original PDB file. This is then a "wet" pocket representation of the active site, and this may be crucial for conduit of protons from the outside in. Interestingly, the pKa of the Arg substrate in the "wet" site is 7.98 (calculated by PROPKA) ${ }^{\mathrm{S} 8}$, while using the 0 ps snapshot, it is 7.24. The unprocessed crystal structure PDB-file (1NOD) yields 11.64! Since the mechanism of protonation as such was not the focus of the study, the ensuing QM/MM calculations were started from the 0 ps snapshot.

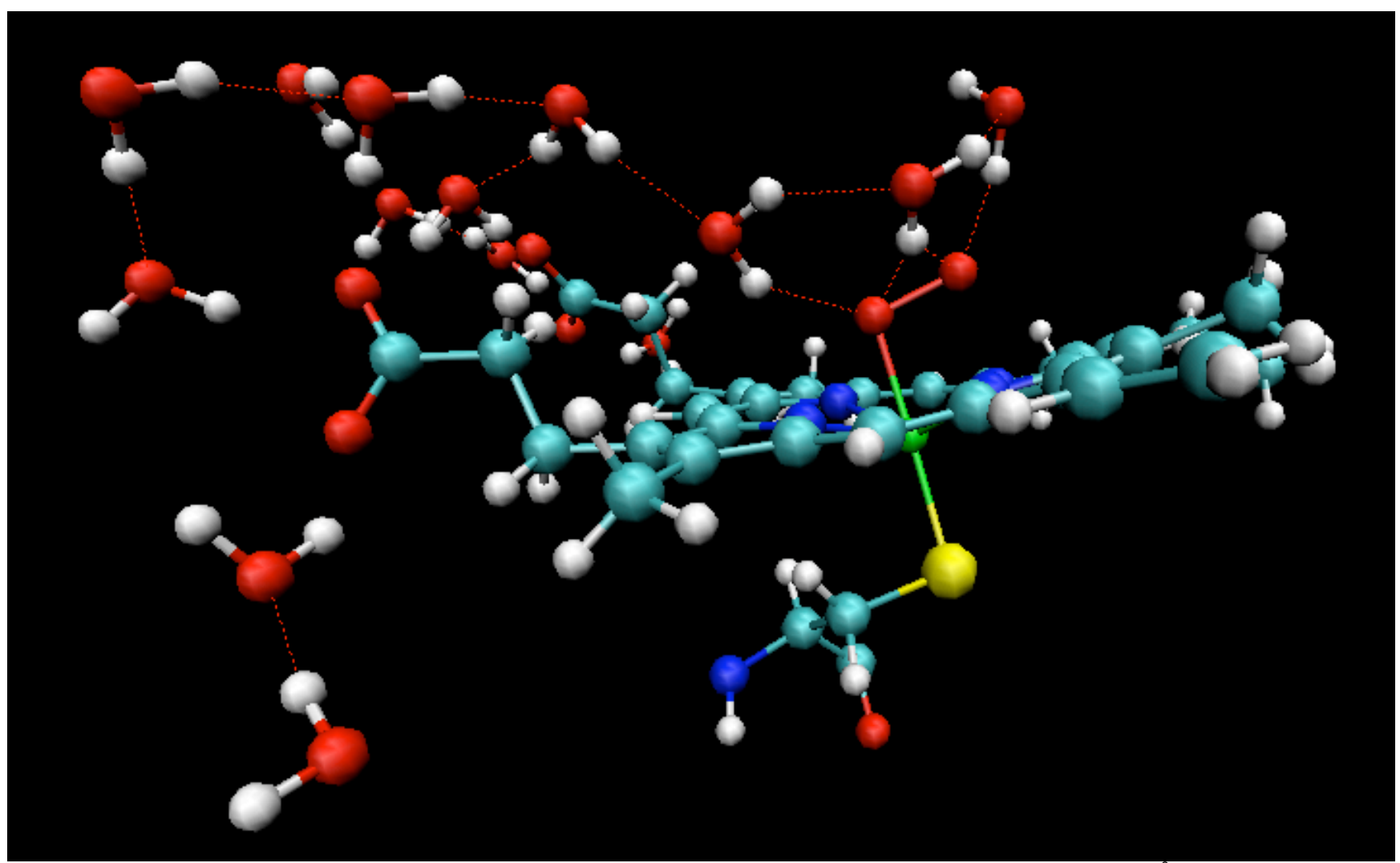

Figure S1. Snapshot 293ps from an MD simulation. Water molecules closer than $4.4 \AA$ from the heme is shown, and their hydrogen bonds are highlighted. Enzyme residues have been removed for clarity. 


\section{Reaction 1}

Total QM charge: 0

No. of QM atoms: 93

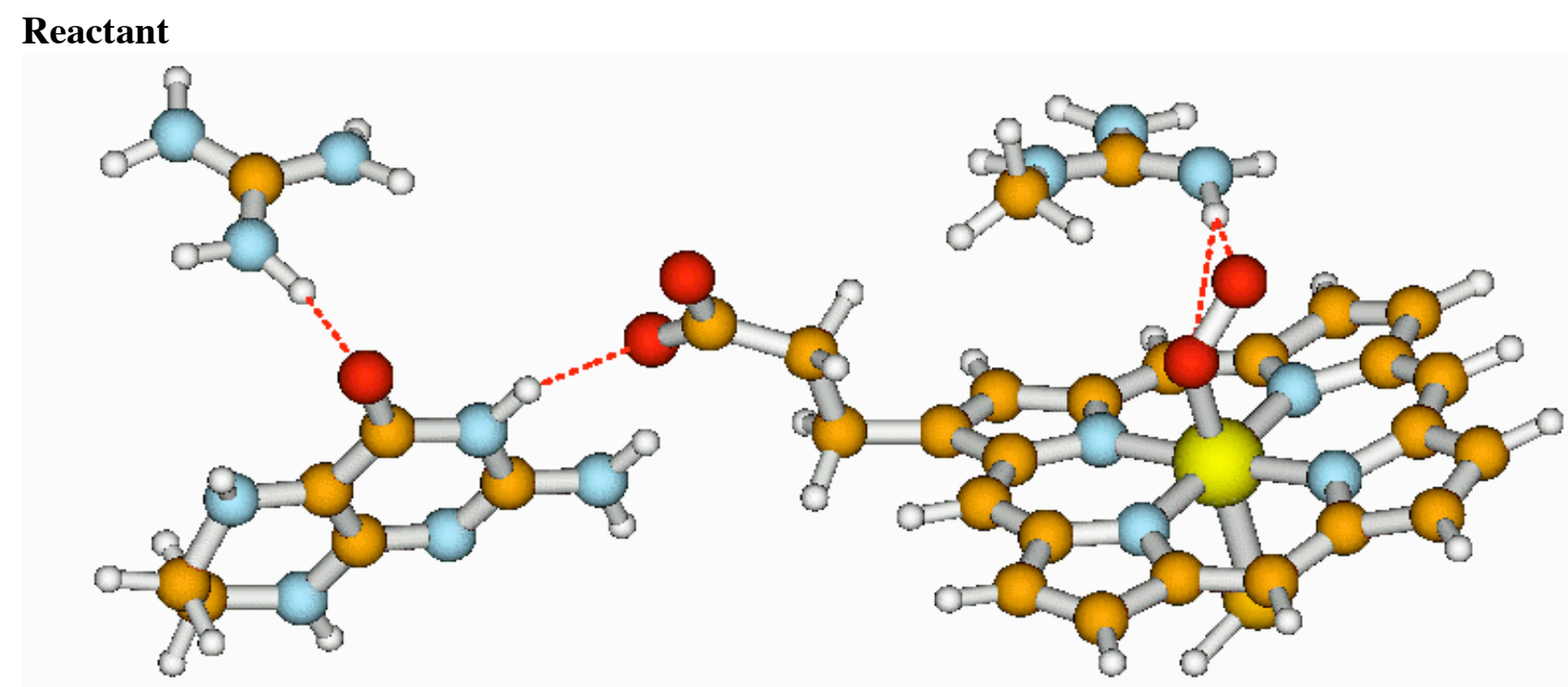

Table S1.1.1. Geometries ( $)$.

\begin{tabular}{|l|c|c|c|c|}
\hline & Singlet & Triplet & Quintet & Septet \\
\hline $\mathrm{Fe}-\mathrm{O}_{\text {inner }}$ & 2.02 & 2.07 & 2.44 & 2.40 \\
\hline $\mathrm{Fe}-\mathrm{S}$ & 2.42 & 2.40 & 2.62 & 2.56 \\
\hline $\mathrm{O}_{\text {inner }}-\mathrm{O}_{\text {outer }}$ & 1.37 & 1.38 & 1.38 & 1.38 \\
\hline $\mathrm{ArgH}-\mathrm{O}_{\text {outer }}$ & 1.69 & 1.66 & 1.60 & 1.62 \\
\hline PropionO $-\mathrm{H}_{4} \mathrm{~B}$ & 1.92 & 1.92 & 1.93 & 1.92 \\
\hline
\end{tabular}

Table S1.1.2. Valence electron configurations.

\begin{tabular}{|c|c|c|c|}
\hline Singlet & Triplet & Quintet & Septet \\
\hline$\alpha\left(\pi_{\mathrm{yz}}^{*}\right) \beta\left(\pi_{\mathrm{OO}}^{*}\right)$ & $\alpha\left(\pi_{\mathrm{yz}}^{*} \pi_{\mathrm{OO}}^{*}\right)$ & $\alpha\left(\pi_{\mathrm{xz}}^{*} \pi_{\mathrm{yz}}^{*} \pi_{\mathrm{OO}}^{*} \sigma_{\mathrm{z} 2}^{*}\right)$ & $\alpha\left(\delta \pi_{\mathrm{xz}}^{*} \pi_{\mathrm{yz}}^{*} \pi_{\mathrm{OO}}^{*} \sigma_{\mathrm{zz}}^{*} \sigma_{\mathrm{xy}}^{*}\right)$ \\
\hline
\end{tabular}


Table S1.1.3. Spin and charge distributions and expectation value $<\mathrm{S}^{2}>$. Data from $\mathrm{B} 2$ calculations.

\begin{tabular}{|c|c|c|c|c|c|c|c|c|}
\hline & \multicolumn{2}{|c|}{ Singlet } & \multicolumn{2}{c|}{ Triplet } & \multicolumn{2}{c|}{ Quintet } & \multicolumn{2}{c|}{ Septet } \\
\hline & Spin & Charge & Spin & Charge & Spin & Charge & Spin & Charge \\
\hline $\mathrm{Fe}$ & 1.15 & 0.19 & 1.10 & 0.16 & 2.75 & 0.12 & 4.18 & 0.25 \\
\hline $\mathrm{O}_{\text {inner }}$ & -0.53 & -0.01 & 0.49 & -0.02 & 0.60 & -0.08 & 0.61 & -0.04 \\
\hline $\mathrm{O}_{\text {outer }}$ & -0.48 & -0.41 & 0.47 & -0.42 & 0.45 & -0.47 & 0.46 & -0.47 \\
\hline $\mathrm{S}$ & -0.02 & -0.68 & -0.02 & -0.65 & 0.26 & -0.70 & 0.28 & -0.63 \\
\hline $\mathrm{Heme}$ & -0.10 & -1.26 & -0.04 & -1.24 & -0.05 & -0.98 & 0.47 & -1.24 \\
\hline $\mathrm{Arg}_{\text {substrate }}$ & -0.01 & 0.96 & 0.01 & 0.96 & 0.00 & 0.92 & 0.00 & 0.93 \\
\hline $\mathrm{H}_{4} \mathrm{~B}$ & 0.00 & 0.08 & 0.00 & 0.08 & 0.00 & 0.08 & 0.00 & 0.08 \\
\hline$<\mathrm{S}^{2}>$ & \multicolumn{2}{|c|}{1.04} & \multicolumn{2}{c|}{2.07} & & & & 12.02 \\
\hline
\end{tabular}

Table S1.1.4. Relative energies of the different multiplicities (kcal $/ \mathrm{mol})$.

\begin{tabular}{|c|c|c|c|c|c|c|}
\hline & \multicolumn{4}{|c|}{ QM part of QM/MM energy } & \multicolumn{3}{c|}{ Total QM/MM energy } \\
\hline & B1 & B2 & B2W & B1 & B2 & B2 ${ }^{\mathrm{a}}$ \\
\hline Singlet & $\mathbf{0 . 0 0}$ & $\mathbf{0 . 0 0}$ & $\mathbf{0 . 0 0}$ & $\mathbf{0 . 0 0}$ & $\mathbf{0 . 0 0}$ & $\mathbf{0 . 0 0}$ \\
\hline Triplet & 1.70 & 1.76 & 2.01 & 1.57 & 1.61 & 1.86 \\
\hline Quintet & -0.59 & -0.13 & ---- & 0.91 & 1.39 & --- \\
\hline Septet & 5.96 & 1.85 & ---- & 5.20 & 1.06 & --- \\
\hline
\end{tabular}

${ }^{\mathrm{a}}$ For the purpose of basis set testing. 


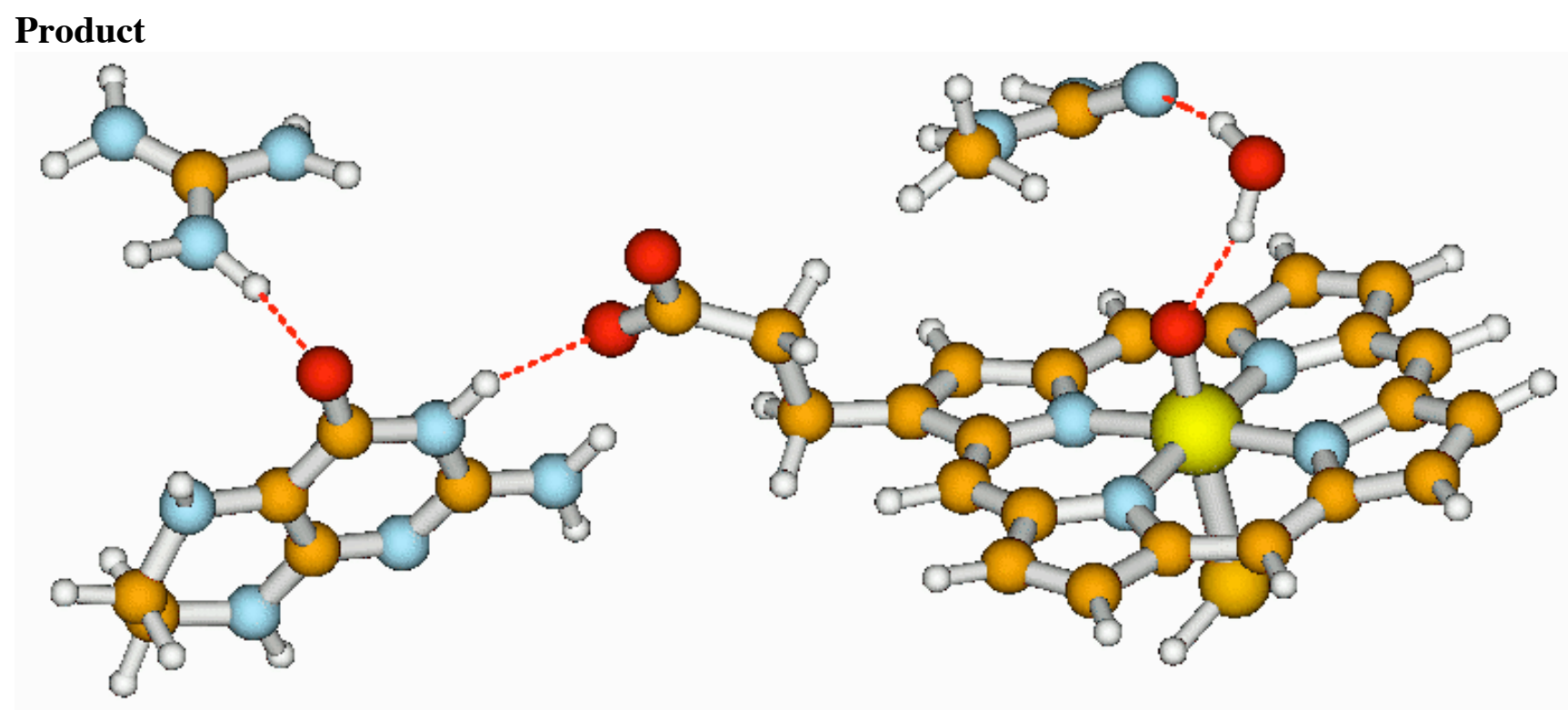

Table S1.2.1. Geometries ( $\AA$ ).

\begin{tabular}{|l|c|c|c|c|}
\hline & Singlet & Triplet & Quintet & Septet \\
\hline $\mathrm{Fe}-\mathrm{O}_{\text {inner }}$ & 1.66 & 1.66 & 1.66 & 1.67 \\
\hline $\mathrm{Fe}-\mathrm{S}$ & 2.59 & 2.58 & 2.57 & 2.55 \\
\hline $\mathrm{O}_{\text {inner }}-\mathrm{O}_{\text {outer }}$ & 2.72 & 2.73 & 2.73 & 2.72 \\
\hline ArgH $-\mathrm{O}_{\text {outer }}$ & 1.00 & 1.00 & 1.00 & 1.00 \\
\hline PropionO $-\mathrm{H}_{4} \mathrm{~B}$ & 1.94 & 1.94 & 1.94 & 1.94 \\
\hline
\end{tabular}

Table S1.2.2. Valence electron configurations.

\begin{tabular}{|c|c|c|c|}
\hline Singlet & Triplet & Quintet & Septet \\
\hline$\alpha\left(\pi_{\mathrm{xz}}^{*} \pi_{\mathrm{yz}}^{*}\right) \beta\left(\mathrm{a}_{2 \mathrm{u}} \mathrm{p}_{\mathrm{Nsubs}}\right)$ & $\alpha\left(\pi_{\mathrm{xz}}^{*} \pi_{\mathrm{yz}}^{*} \mathrm{p}_{\mathrm{Nsubs}}\right) \beta\left(\mathrm{a}_{2 \mathrm{u}}\right)$ & $\alpha\left(\pi_{\mathrm{xz}}^{*} \pi_{\mathrm{yz}}^{*} \mathrm{a}_{2 \mathrm{u}} \mathrm{p}_{\mathrm{Nsubs}}\right)$ & $\alpha\left(\delta \pi_{\mathrm{xz}}^{*} \pi_{\mathrm{yz}}^{*} \mathrm{a}_{2 \mathrm{u}} \mathrm{p}_{\mathrm{Nsubs}} \sigma_{\mathrm{xy}}^{*}\right)$ \\
\hline
\end{tabular}


Table S1.2.3. Spin and charge distributions and expectation value $<\mathrm{S}^{2}>$. Data from $\mathrm{B} 2$ calculations.

\begin{tabular}{|c|c|c|c|c|c|c|c|c|}
\hline & \multicolumn{2}{|c|}{ Singlet } & \multicolumn{2}{c|}{ Triplet } & \multicolumn{2}{c|}{ Quintet } & \multicolumn{2}{c|}{ Septet } \\
\hline & Spin & Charge & Spin & Charge & Spin & Charge & Spin & Charge \\
\hline $\mathrm{Fe}$ & 1.39 & 0.27 & 1.38 & 0.26 & 1.23 & 0.26 & 3.24 & 0.38 \\
\hline $\mathrm{O}_{\text {inner }}$ & 0.79 & -0.36 & 0.79 & -0.36 & 0.82 & -0.36 & 0.59 & -0.33 \\
\hline $\mathrm{O}_{\text {outer }}$ & 0.00 & -1.04 & 0.01 & -1.05 & 0.01 & -1.04 & 0.01 & -1.04 \\
\hline $\mathrm{S}$ & -0.22 & -0.74 & -0.22 & -0.74 & 0.17 & -0.73 & 0.02 & -0.73 \\
\hline $\mathrm{Heme}$ & -0.90 & -0.47 & -0.94 & -0.44 & 0.76 & -0.45 & 1.12 & -0.60 \\
\hline $\mathrm{Arg}_{\text {substrate }}$ & -1.05 & 1.13 & 0.98 & 1.10 & 1.01 & 1.11 & 1.03 & 1.12 \\
\hline $\mathrm{H}_{4} \mathrm{~B}$ & 0.00 & 0.08 & 0.00 & 0.08 & 0.00 & 0.08 & 0.00 & 0.08 \\
\hline$<\mathrm{S}^{2}>$ & \multicolumn{2}{|c|}{2.07} & \multicolumn{2}{|c|}{3.07} & \multicolumn{2}{c|}{6.07} & & 12.15 \\
\hline
\end{tabular}

Table S1.2.4. Energies (relative to Table S1.1.4) of the different multiplicities ( $\mathrm{kcal} / \mathrm{mol})$.

\begin{tabular}{|c|c|c|c|c|c|c|}
\hline & \multicolumn{4}{|c|}{ QM part of QM/MM energy } & \multicolumn{3}{c|}{ Total QM/MM energy } \\
\hline & B1 & B2 & B2W & B1 & B2 & B2 ${ }^{\mathrm{a}}$ \\
\hline Singlet & 20.55 & 16.30 & 10.64 & 27.06 & 22.77 & 17.1 \\
\hline Triplet & 21.27 & 16.73 & ---- & 27.28 & 22.72 & ---- \\
\hline Quintet & 20.90 & 16.71 & ---- & 27.06 & 22.88 & --- \\
\hline Septet & 34.66 & 26.92 & ---- & 39.64 & 31.91 & ---- \\
\hline
\end{tabular}

${ }^{\mathrm{a}}$ For the purpose of basis set testing. 


\section{Reaction 2}

Total QM charge: +1

No. of QM atoms: 94

\section{Reactant}

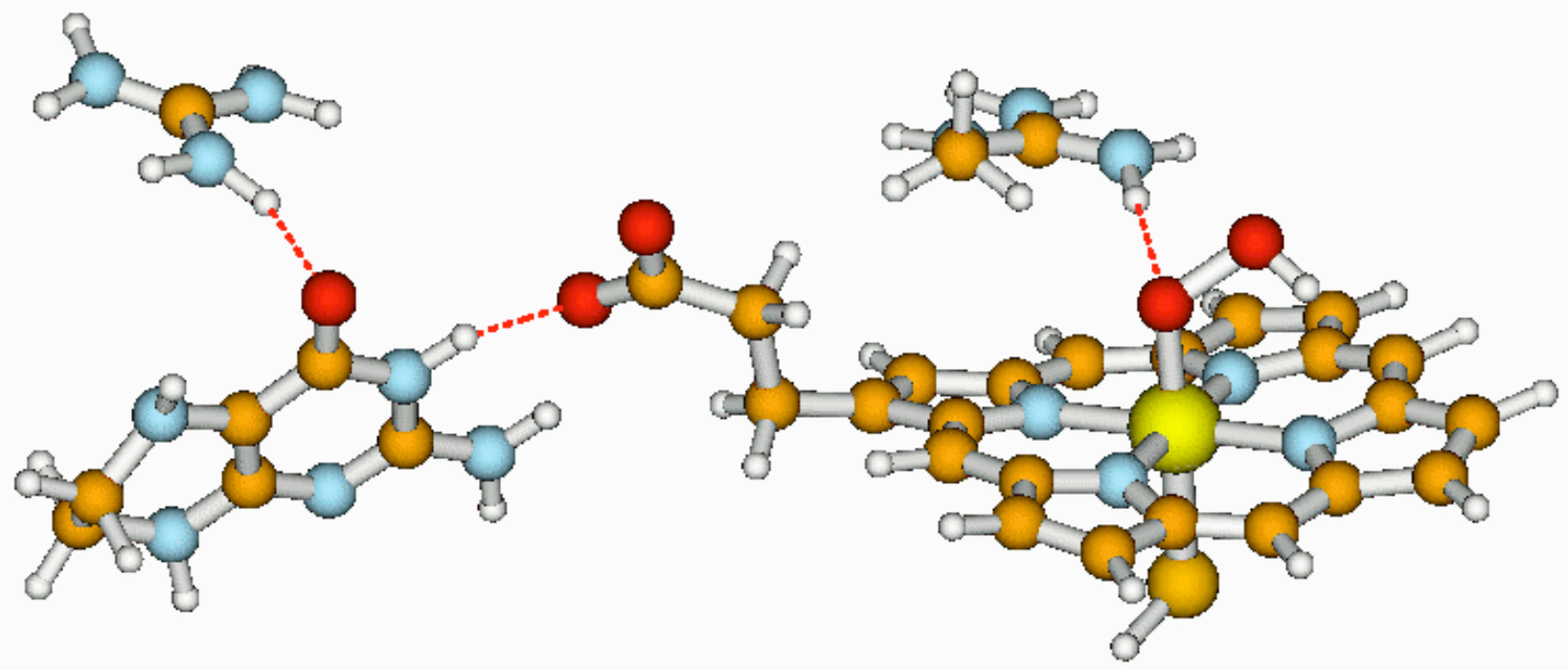

Table S2.1.1. Geometries $(\AA)$.

\begin{tabular}{|l|c|c|c|c|}
\hline & Singlet & Triplet & Quintet & Septet \\
\hline $\mathrm{Fe}-\mathrm{O}_{\text {inner }}$ & 1.92 & 1.92 & 2.08 & 2.04 \\
\hline $\mathrm{Fe}-\mathrm{S}$ & 2.46 & 2.45 & 2.86 & 2.78 \\
\hline $\mathrm{O}_{\text {inner }}-\mathrm{O}_{\text {outer }}$ & 1.53 & 1.53 & 1.52 & 1.52 \\
\hline ArgH $-\mathrm{O}_{\text {outer }}$ & 2.09 & 2.09 & 2.17 & 2.20 \\
\hline PropionO $-\mathrm{H}_{4} \mathrm{~B}$ & 1.83 & 1.83 & 1.84 & 1.83 \\
\hline
\end{tabular}

Table S2.1.2. Valence electron configurations.

\begin{tabular}{|c|c|c|c|}
\hline Singlet $^{\mathrm{a}}$ & Triplet $^{\mathrm{b}}$ & Quintet & Septet $^{*}$ \\
\hline$\alpha\left(\pi_{\mathrm{yz}}^{*}\right) \beta\left(\mathrm{a}_{2 \mathrm{u}}\right)$ & $\alpha\left(\pi_{\mathrm{yz}}^{*} \mathrm{a}_{2 \mathrm{u}}\right)$ & $\alpha\left(\pi_{\mathrm{xz}}^{*} \pi_{\mathrm{yz}}^{*} \mathrm{a}_{2 \mathrm{u}} \sigma_{\mathrm{z} 2}^{*}\right)$ & $\alpha\left(\delta \pi_{\mathrm{xz}}^{*}{\pi_{\mathrm{yz}}^{*}}^{*} \mathrm{a}_{2 \mathrm{u}} \sigma_{\mathrm{zz}}^{*} \sigma_{\mathrm{xy}}^{*}\right)$ \\
\hline
\end{tabular}

${ }^{a}$ A closed shell configuration was found to be at 20.26/19.30 (QM/Total) kcal/mol at B1 level.

${ }^{\mathrm{b}}$ A configuration with $\alpha\left(\pi_{\mathrm{yz}}^{*} \pi_{\mathrm{xz}}{ }^{*}\right)$ was found to be at 6.01/5.01 (QM/Total) kcal/mol at B1 level. 
Table S2.1.3. Spin and charge distributions and expectation value $<\mathrm{S}^{2}>$. Data from $\mathrm{B} 2$ calculations.

\begin{tabular}{|c|c|c|c|c|c|c|c|c|}
\hline & \multicolumn{2}{|c|}{ Singlet } & \multicolumn{2}{c|}{ Triplet } & \multicolumn{2}{c|}{ Quintet } & \multicolumn{2}{c|}{ Septet } \\
\hline & Spin & Charge & Spin & Charge & Spin & Charge & Spin & Charge \\
\hline $\mathrm{Fe}$ & 1.14 & 0.13 & 0.98 & 0.12 & 2.70 & 0.25 & 4.16 & 0.34 \\
\hline $\mathrm{O}_{\text {inner }}$ & 0.00 & -0.20 & 0.09 & -0.20 & 0.22 & -0.26 & 0.22 & -0.21 \\
\hline $\mathrm{O}_{\text {outer }}$ & -0.01 & -0.50 & 0.00 & -0.50 & 0.03 & -0.48 & 0.03 & -0.47 \\
\hline $\mathrm{S}$ & -0.15 & -0.56 & 0.08 & -0.56 & 0.55 & -0.53 & 0.51 & -0.54 \\
\hline $\mathrm{Heme}$ & -0.92 & -0.66 & 0.85 & -0.59 & 0.51 & -0.67 & 1.08 & -0.84 \\
\hline $\mathrm{Arg}_{\text {substrate }}$ & 0.00 & 1.02 & 0.00 & 1.02 & 0.00 & 1.00 & 0.00 & 1.00 \\
\hline $\mathrm{H}_{4} \mathrm{~B}$ & -0.06 & 0.12 & 0.00 & 0.06 & 0.00 & 0.06 & 0.00 & 0.06 \\
\hline$<\mathrm{S}^{2}>$ & \multicolumn{2}{|c|}{1.10} & \multicolumn{2}{|c|}{2.07} & \multicolumn{2}{c|}{6.05} & & 12.04 \\
\hline
\end{tabular}

Table S2.1.4. Relative energies of the different multiplicities (kcal $/ \mathrm{mol})$.

\begin{tabular}{|c|c|c|c|c|c|c|}
\hline & \multicolumn{4}{|c|}{ QM part of QM/MM energy } & \multicolumn{3}{c|}{ Total QM/MM energy } \\
\hline & B1 & B2 & B2W & B1 & B2 & B2 ${ }^{\mathrm{a}}$ \\
\hline Singlet & $\mathbf{0 . 0 0}$ & $\mathbf{0 . 0 0}$ & $\mathbf{0 . 0 0}$ & $\mathbf{0 . 0 0}$ & $\mathbf{0 . 0 0}$ & $\mathbf{0 . 0 0}$ \\
\hline Triplet & 0.61 & 0.51 & ---- & 0.56 & 0.42 & ---- \\
\hline Quintet & 5.53 & 5.63 & ---- & 5.15 & 5.32 & --- \\
\hline Septet & 9.24 & 4.68 & ---- & 8.06 & 3.53 & --- \\
\hline
\end{tabular}

${ }^{a}$ For the purpose of basis set testing. 


\section{Product}

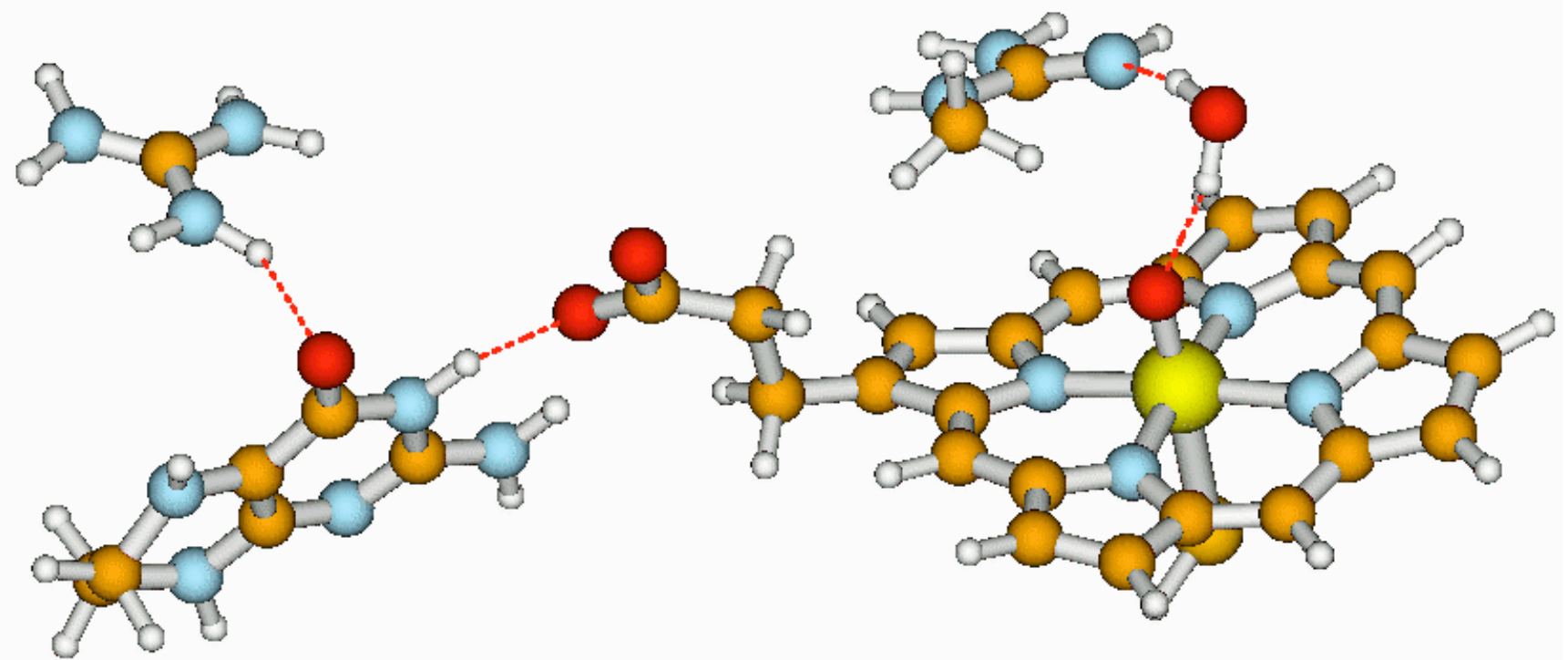

Table S2.2.1. Geometries ( $)$.

\begin{tabular}{|l|c|c|c|c|}
\hline & Singlet & Triplet & Quintet & Septet \\
\hline $\mathrm{Fe}-\mathrm{O}_{\text {inner }}$ & 1.65 & 1.65 & 1.66 & 1.67 \\
\hline $\mathrm{Fe}-\mathrm{S}$ & 2.58 & 2.58 & 2.56 & 2.53 \\
\hline $\mathrm{O}_{\text {inner }}-\mathrm{O}_{\text {outer }}$ & 2.68 & 2.69 & 2.68 & 2.68 \\
\hline $\mathrm{ArgH}-\mathrm{O}_{\text {outer }}$ & 0.99 & 1.00 & 0.99 & 0.99 \\
\hline PropionO $-\mathrm{H}_{4} \mathrm{~B}$ & 1.78 & 1.79 & 1.78 & 1.77 \\
\hline
\end{tabular}

Table S1.2.2. Valence electron configurations.

\begin{tabular}{|c|c|c|c|}
\hline Singlet & Triplet & Quintet & Septet \\
\hline$\alpha\left(\pi_{x z}^{*} \pi_{y z}^{*}\right) \beta\left(a_{2 u} \pi_{\text {substHAB }}\right)$ & $\alpha\left(\pi_{\mathrm{xz}}^{*} \pi_{\mathrm{yz} z}^{*} \pi_{\mathrm{subs}+\mathrm{H} 4 \mathrm{~B}}\right) \beta\left(\mathrm{a}_{2 \mathrm{u}}\right)$ & $\alpha\left(\pi_{x z}^{*} \pi_{y z}^{*} a_{2 u} \pi_{\text {subss }+H A B}\right)$ & $\alpha\left(\delta \pi_{x z}^{*} \pi_{y z}^{*} a_{2 u} \pi_{s u b s+H A B} \sigma_{x y}^{*}\right)$ \\
\hline
\end{tabular}

Table S2.2.3. Spin and charge distributions and expectation value $\left\langle\mathrm{S}^{2}\right\rangle$. Data from $\mathrm{B} 2$ calculations.

\begin{tabular}{|c|c|c|c|c|c|c|c|c|}
\hline & \multicolumn{2}{|c|}{ Singlet } & \multicolumn{2}{c|}{ Triplet } & \multicolumn{2}{c|}{ Quintet } & \multicolumn{2}{c|}{ Septet } \\
\hline & Spin & Charge & Spin & Charge & Spin & Charge & Spin & Charge \\
\hline $\mathrm{Fe}$ & 1.41 & 0.25 & 1.40 & 0.25 & 1.24 & 0.25 & 3.24 & 0.36 \\
\hline $\mathrm{O}_{\text {inner }}$ & 0.76 & -0.40 & 0.77 & -0.39 & 0.80 & -0.40 & 0.58 & -0.39 \\
\hline $\mathrm{O}_{\text {outer }}$ & 0.00 & -0.94 & 0.01 & -0.94 & 0.01 & -0.94 & 0.00 & -0.95 \\
\hline $\mathrm{S}$ & -0.30 & -0.61 & -0.29 & -0.61 & 0.26 & -0.60 & 0.10 & -0.59 \\
\hline $\mathrm{Heme}$ & -1.12 & -0.26 & -0.51 & -0.15 & 0.89 & -0.31 & 1.31 & -0.40 \\
\hline $\mathrm{Arg}_{\text {substrate }}$ & -0.27 & 0.80 & 0.21 & 0.75 & 0.31 & 0.83 & 0.24 & 0.77 \\
\hline $\mathrm{H}_{4} \mathrm{~B}$ & -0.48 & 0.49 & 0.43 & 0.44 & 0.50 & 0.51 & 0.53 & 0.54 \\
\hline$<\mathrm{S}^{2}>$ & \multicolumn{3}{|c|}{2.06} & \multicolumn{3}{|c|}{3.09} & \multicolumn{3}{c|}{6.06} & \multicolumn{2}{c|}{12.14} \\
\hline
\end{tabular}


Table S2.2.4. Energies (relative to Table S2.1.4) of the different multiplicities (kcal/mol).

\begin{tabular}{|c|c|c|c|c|c|c|}
\hline & \multicolumn{4}{|c|}{ QM part of QM/MM energy } & \multicolumn{3}{c|}{ Total QM/MM energy } \\
\hline & B1 & B2 & B2W & B1 & B2 & B2 ${ }^{\mathrm{a}}$ \\
\hline Singlet & 22.87 & 15.90 & 13.81 & 20.85 & 13.83 & 11.74 \\
\hline Triplet & 22.64 & 15.25 & ---- & 19.80 & 12.40 & ---- \\
\hline Quintet & 23.21 & 16.51 & ---- & 20.92 & 14.29 & ---- \\
\hline Septet & 36.71 & 27.64 & ---- & 34.52 & 25.47 & --- \\
\hline
\end{tabular}

${ }^{\mathrm{a}}$ For the purpose of basis set testing. 


\section{Reaction 3}

Total QM charge: +2

No. of QM atoms: 95

\section{Reactant}

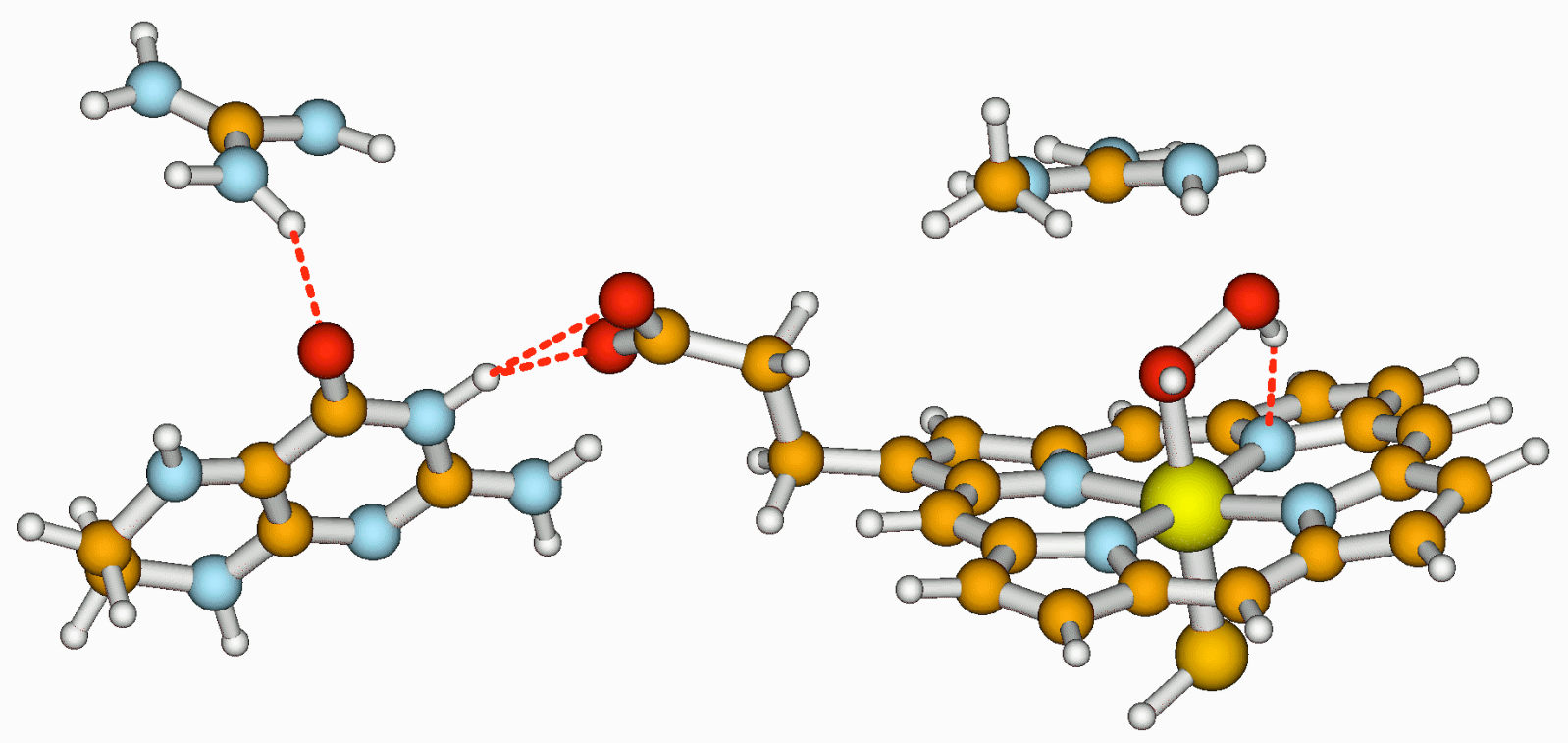

A)

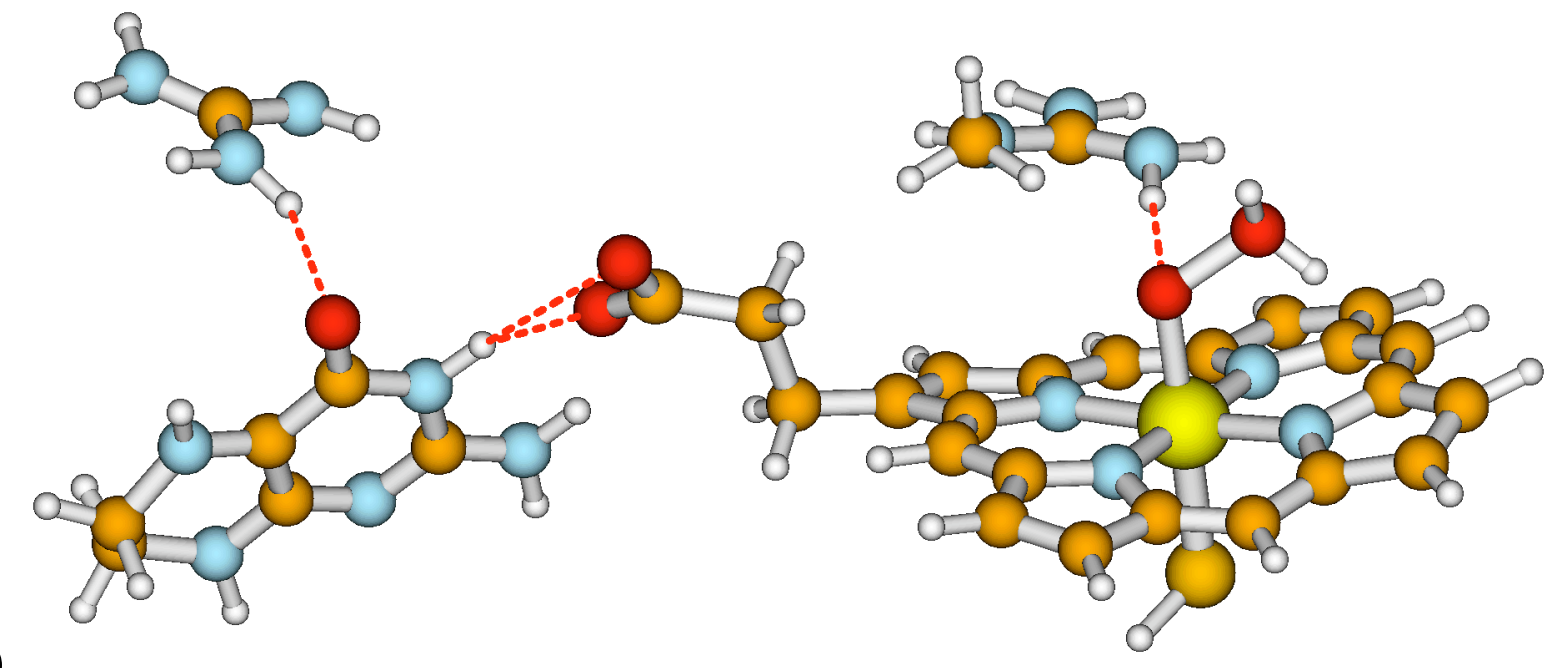

B)

All the triplet, quintet and septet structures are calculated on the A form.

Table S3.1.1. Geometries $(\AA)$.

\begin{tabular}{|l|c|c|c|c|c|}
\hline & Singlet $(\boldsymbol{A})$ & Singlet $(\boldsymbol{B})$ & Triplet & Quintet & Septet \\
\hline $\mathrm{Fe}-\mathrm{O}_{\text {inner }}$ & 2.28 & 2.03 & 2.68 & 2.70 & 2.89 \\
\hline $\mathrm{Fe}-\mathrm{S}$ & 2.33 & 2.38 & 2.47 & 2.48 & 2.40 \\
\hline $\mathrm{O}_{\text {inner }}-\mathrm{O}_{\text {outer }}$ & 1.53 & 1.62 & 1.52 & 1.52 & 1.51 \\
\hline ArgH $-\mathrm{O}_{\text {outer }}$ & 2.05 & $1.88^{\mathrm{a}}$ & 2.10 & 2.09 & 2.24 \\
\hline PropionO $-\mathrm{H}_{4} \mathrm{~B}$ & 1.79 & 1.81 & 1.81 & 1.79 & 1.79 \\
\hline
\end{tabular}

${ }^{\mathrm{a}} \mathrm{ArgH}-\mathrm{O}_{\text {inner }}$ distance. 
Table S3.1.2. Valence electron configurations.

\begin{tabular}{|c|c|c|c|c|}
\hline Singlet (A) & Singlet (B) & Triplet $^{\mathrm{a}}$ & Quintet $^{\mathrm{b}}$ & Septet \\
\hline$\alpha\left(\pi_{y z}^{*}\right) \beta\left(\mathrm{H}_{4} \mathrm{~B}\right)$ & $\alpha\left(\pi_{y z}^{*}\right) \beta\left(\mathrm{H}_{4} \mathrm{~B}+\mathrm{a}_{2 u}\right)$ & $\alpha\left(\pi_{y z}^{*} \pi_{x z}^{*} \sigma_{z 2}^{*}\right) \beta\left(\mathrm{H}_{4} \mathrm{~B}\right)$ & $\alpha\left(\delta \pi_{y z}^{*} \pi_{x z}^{*} \sigma_{z 2}^{*} \sigma_{x y}^{*}\right) \beta\left(\mathrm{H}_{4} \mathrm{~B}\right)$ & $\alpha\left(\delta \pi_{y z}^{*} \pi_{x z}^{*} \sigma_{z 2}^{*} \sigma_{x y}^{*} \mathrm{H}_{4} \mathrm{~B}\right)$ \\
\hline
\end{tabular}

${ }^{a}$ A configuration with $\alpha\left(\pi_{\mathrm{yz}}^{*} \mathrm{H}_{4} \mathrm{~B}\right)$ was found to be at $-0.16 /-0.13(\mathrm{QM} / \mathrm{Total}) \mathrm{kcal} / \mathrm{mol}$ at B1 level.

${ }^{\mathrm{b}}$ A configuration with $\alpha\left(\pi_{\mathrm{yz}}^{*} \pi_{\mathrm{xz}}^{*} \sigma_{\mathrm{z} 2}^{*} \mathrm{H}_{4} \mathrm{~B}\right)$ was found to be at $-4.38 /-1.71$ (QM/Total) kcal/mol at B1 level, but was subsequently found to be higher at $\mathrm{B} 2$ level than the one presented.

Table S3.1.3. Spin and charge distributions and expectation value $\left\langle\mathrm{S}^{2}\right\rangle$. Data from B2 calculations.

\begin{tabular}{|c|c|c|c|c|c|c|c|c|c|c|}
\hline & \multicolumn{2}{|c|}{ Singlet $(\boldsymbol{A})$} & \multicolumn{2}{c|}{ Singlet $(\boldsymbol{B})$} & \multicolumn{2}{c|}{ Triplet } & \multicolumn{2}{c|}{ Quintet } & \multicolumn{2}{c|}{ Septet } \\
\hline & Spin & Charge & Spin & Charge & Spin & Charge & Spin & Charge & Spin & Charge \\
\hline $\mathrm{Fe}$ & 1.18 & 0.02 & 1.16 & 0.09 & 2.69 & 0.03 & 2.68 & 0.06 & 4.12 & 0.07 \\
\hline $\mathrm{O}_{\text {inner }}$ & -0.01 & -0.28 & 0.01 & -0.20 & -0.01 & -0.28 & -0.01 & -0.29 & 0.00 & -0.32 \\
\hline $\mathrm{O}_{\text {outer }}$ & 0.00 & -0.42 & -0.01 & -0.51 & 0.00 & -0.44 & 0.00 & -0.45 & 0.00 & -0.47 \\
\hline $\mathrm{S}$ & -0.08 & -0.34 & -0.07 & -0.48 & 0.28 & -0.44 & 0.38 & -0.48 & 0.39 & -0.37 \\
\hline $\mathrm{Heme}$ & -0.29 & -0.89 & -0.39 & -0.90 & -0.19 & -0.73 & 0.12 & -0.76 & 0.62 & -0.95 \\
\hline $\mathrm{Arg}_{\text {substrate }}$ & 0.00 & 1.01 & 0.00 & 1.01 & 0.00 & 1.02 & 0.00 & 1.02 & 0.00 & 1.00 \\
\hline $\mathrm{H}_{4} \mathrm{~B}$ & -0.80 & 0.76 & -0.70 & 0.67 & -0.77 & 0.73 & 0.84 & 0.80 & 0.87 & 0.83 \\
\hline$<\mathrm{S}^{2}>$ & \multicolumn{2}{|c|}{1.13} & \multicolumn{6}{|c|}{1.09} & \multicolumn{6}{|c|}{2.96} & \multicolumn{3}{c|}{6.05} & \multicolumn{2}{c|}{12.02} \\
\hline
\end{tabular}

Table S3.1.4. Relative energies of the different multiplicities ( $\mathrm{kcal} / \mathrm{mol})$.

\begin{tabular}{|c|c|c|c|c|}
\hline & \multicolumn{2}{|c|}{ QM part of QM/MM energy } & \multicolumn{2}{c|}{ Total QM/MM energy } \\
\hline & $\mathrm{B} 1$ & $\mathrm{~B} 2$ & $\mathrm{~B} 1$ & $\mathrm{~B} 2$ \\
\hline Singlet (A) & $\mathbf{0 . 0 0}$ & $\mathbf{0 . 0 0}$ & $\mathbf{0 . 0 0}$ & $\mathbf{0 . 0 0}$ \\
\hline Singlet (B) & 3.17 & 8.50 & 14.89 & 20.18 \\
\hline Triplet & -4.44 & -5.53 & -2.40 & -3.45 \\
\hline Quintet & -0.33 & -6.19 & -0.28 & -6.11 \\
\hline Septet & -0.34 & -5.79 & -0.78 & -6.22 \\
\hline
\end{tabular}




\section{Product}

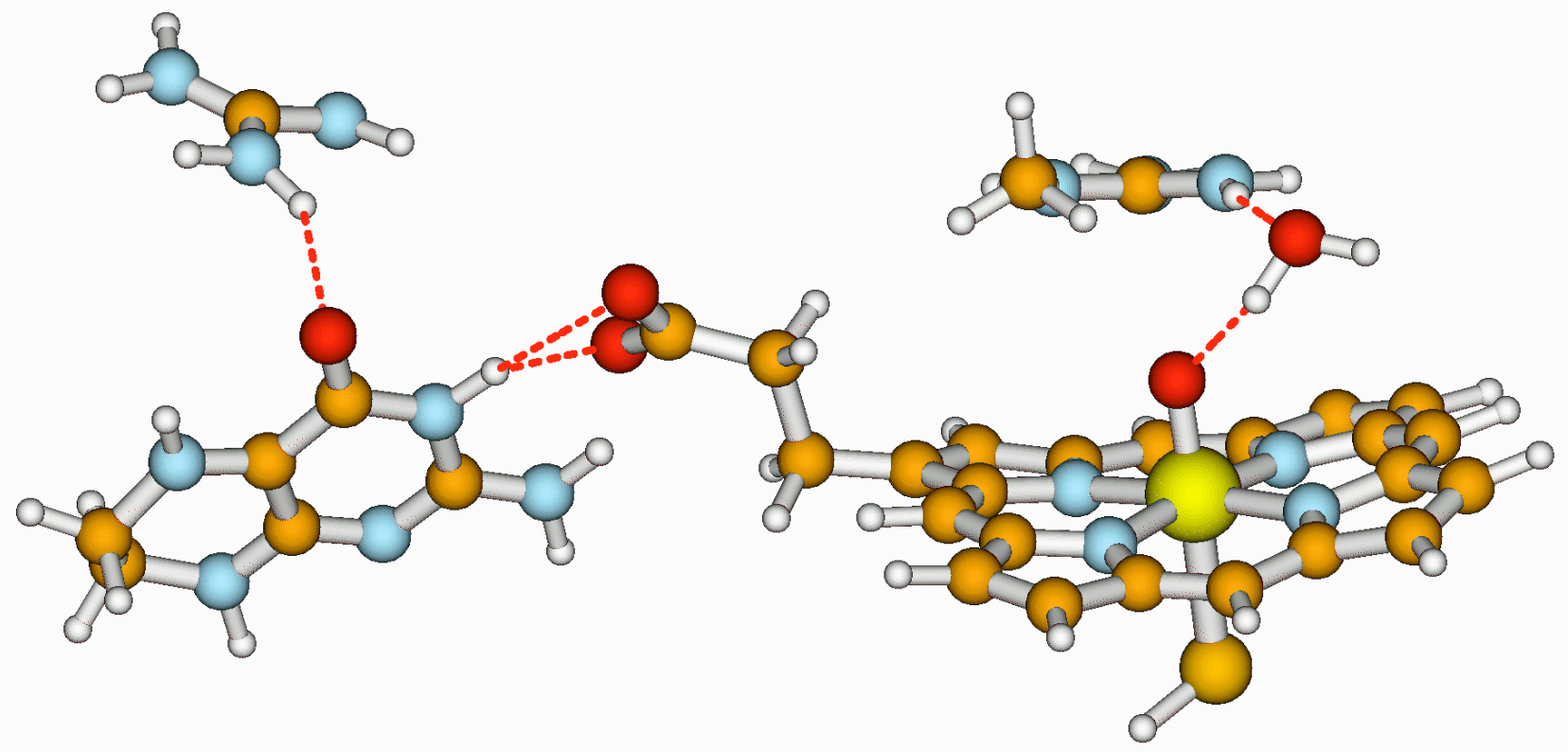

Table S3.2.1. Geometries $(\AA)$.

\begin{tabular}{|l|c|c|c|c|}
\hline & Singlet & Triplet & Quintet & Septet \\
\hline $\mathrm{Fe}-\mathrm{O}_{\text {inner }}$ & 1.67 & 1.67 & 1.67 & 1.68 \\
\hline $\mathrm{Fe}-\mathrm{S}$ & 2.57 & 2.57 & 2.54 & 2.51 \\
\hline $\mathrm{O}_{\text {inner }}-\mathrm{O}_{\text {outer }}$ & 2.56 & 2.57 & 2.57 & 2.56 \\
\hline $\mathrm{ArgH}-\mathrm{O}_{\text {outer }}$ & 1.84 & 1.84 & 1.85 & 1.84 \\
\hline PropionO $-\mathrm{H}_{4} \mathrm{~B}$ & 1.76 & 1.76 & 1.75 & 1.74 \\
\hline
\end{tabular}

Table S3.2.2. Valence electron configurations.

\begin{tabular}{|c|c|c|c|}
\hline Singlet & Triplet $^{\mathrm{a}}$ & Quintet $^{\mathrm{b}}$ & Septet \\
\hline$\alpha\left(\pi_{\mathrm{xz}}^{*} \pi_{\mathrm{yz}}^{*}\right) \beta\left(\mathrm{a}_{2 \mathrm{u}} \mathrm{H}_{4} \mathrm{~B}\right)$ & $\alpha\left(\pi_{\mathrm{xz}}^{*} \pi_{\mathrm{yz}}^{*} \mathrm{H}_{4} \mathrm{~B}\right) \beta\left(\mathrm{a}_{2 \mathrm{u}}\right)$ & $\alpha\left(\pi_{\mathrm{xz}}^{*} \pi_{\mathrm{yz}}^{*} \mathrm{a}_{2 \mathrm{u}} \mathrm{H}_{4} \mathrm{~B}\right)$ & $\alpha\left(\delta \pi_{\mathrm{xz}}^{*} \pi_{\mathrm{yz}}^{*} \mathrm{a}_{2 \mathrm{u}} \mathrm{H}_{4} \mathrm{~B} \sigma_{\mathrm{xy}}^{*}\right)$ \\
\hline
\end{tabular}

${ }^{a}$ A configuration with $\alpha\left(\pi^{*}{ }_{x z} \pi^{*}{ }_{y z} a_{2 u}\right) \beta\left(H_{4} B\right)$ was found to be at $-37.00 /-27.63$ (QM/Total) kcal/mol at B1 level.

${ }^{\mathrm{b}}$ A configuration with $\alpha\left(\delta \pi_{\mathrm{xz}}^{*} \pi_{\mathrm{yz}}^{*} \mathrm{a}_{2 \mathrm{u}} \sigma_{\mathrm{xy}}^{*}\right) \beta\left(\mathrm{H}_{4} \mathrm{~B}\right)$ was found to be at $-22.18 /-13.83$ (QM/Total) $\mathrm{kcal} / \mathrm{mol}$ at B1 level. 
Table S3.2.3. Spin and charge distributions and expectation value $<\mathrm{S}^{2}>$. Data from $\mathrm{B} 2$ calculations.

\begin{tabular}{|c|c|c|c|c|c|c|c|c|}
\hline & \multicolumn{2}{|c|}{ Singlet } & \multicolumn{2}{c|}{ Triplet } & \multicolumn{2}{c|}{ Quintet } & \multicolumn{2}{c|}{ Septet } \\
\hline & Spin & Charge & Spin & Charge & Spin & Charge & Spin & Charge \\
\hline $\mathrm{Fe}$ & 1.52 & 0.29 & 1.52 & 0.28 & 1.33 & 0.28 & 3.31 & 0.34 \\
\hline $\mathrm{O}_{\text {inner }}$ & 0.67 & -0.46 & 0.67 & -0.46 & 0.71 & -0.46 & 0.55 & -0.45 \\
\hline $\mathrm{O}_{\text {outer }}$ & 0.01 & -0.98 & 0.01 & -0.98 & 0.01 & -0.98 & 0.01 & -0.99 \\
\hline $\mathrm{S}$ & -0.32 & -0.57 & -0.33 & -0.56 & 0.26 & -0.57 & -0.03 & -0.57 \\
\hline $\mathrm{Heme}$ & -0.89 & -0.41 & -0.81 & -0.37 & 0.70 & -0.41 & 1.16 & -0.47 \\
\hline $\mathrm{Arg}_{\text {substrate }}$ & 0.00 & 1.01 & 0.00 & 1.01 & 0.00 & 1.01 & 0.00 & 1.00 \\
\hline $\mathrm{H}_{4} \mathrm{~B}$ & -1.00 & 0.94 & 0.94 & 0.89 & 1.00 & 0.94 & 1.00 & 0.94 \\
\hline$<\mathrm{S}^{2}>$ & \multicolumn{2}{|c|}{2.08} & \multicolumn{2}{c|}{3.08} & & & 12.16 \\
\hline
\end{tabular}

Table S3.2.4. Energies (relative to Table S3.1.4) of the different multiplicities ( $\mathrm{kcal} / \mathrm{mol})$.

\begin{tabular}{|c|c|c|c|c|}
\hline & \multicolumn{2}{|c|}{ QM part of QM/MM energy } & \multicolumn{2}{c|}{ Total QM/MM energy } \\
\hline & B1 & B2 & B1 & B2 \\
\hline Singlet & -32.48 & -30.04 & -26.42 & -22.05 \\
\hline Triplet & -37.21 & -35.07 & -27.79 & -25.72 \\
\hline Quintet & -36.80 & -34.64 & -27.48 & -25.32 \\
\hline Septet & -22.04 & -23.43 & -13.72 & -15.07 \\
\hline
\end{tabular}




\section{Reaction 4}

Total QM charge: 0

No. of QM atoms: 96

\section{Reactant}

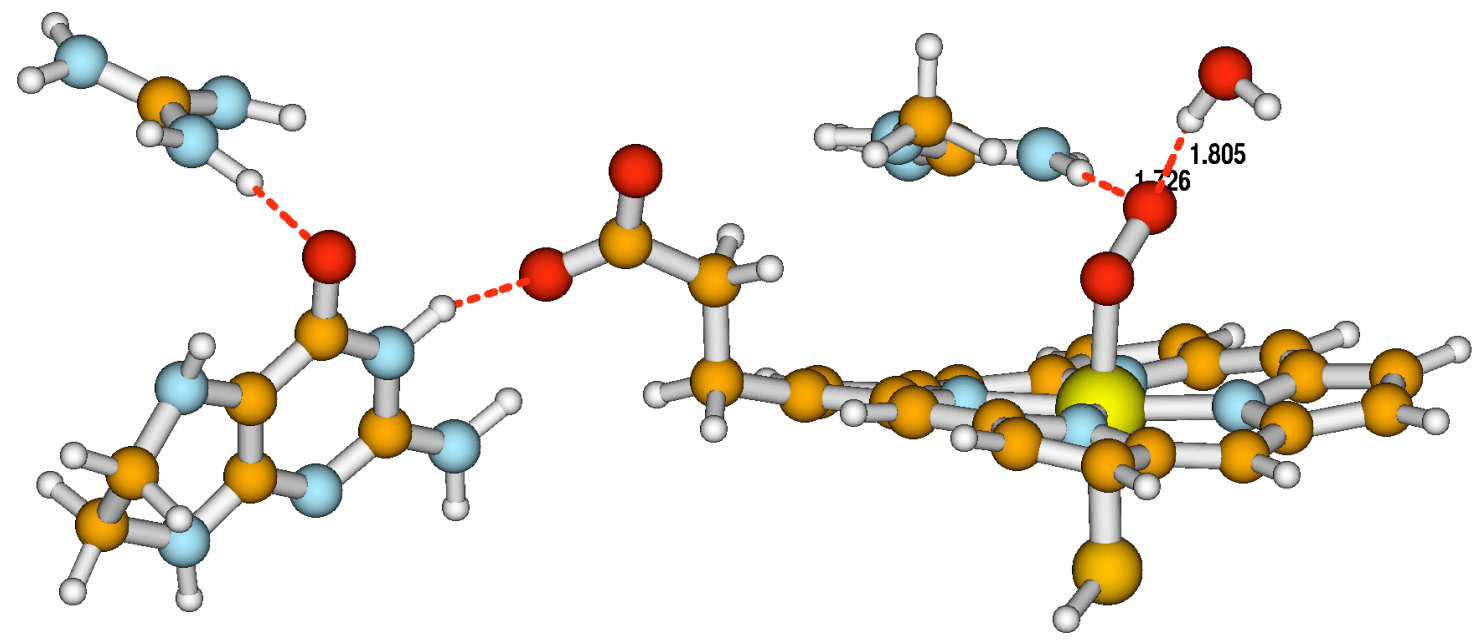

Table S4.1.1. Geometries $(\AA)$.

\begin{tabular}{|c|c|c|c|c|c|}
\hline State & $\boldsymbol{F e}-\boldsymbol{O}_{\text {inner }}$ & $\boldsymbol{F e}-\boldsymbol{S}$ & $\boldsymbol{O}_{\text {inner }}-\boldsymbol{O}_{\text {outer }}$ & ArgH $-\boldsymbol{O}_{\text {outer }}$ & $\boldsymbol{O}_{\text {outer }}-\boldsymbol{H}_{2} \boldsymbol{O}$ \\
\hline$\alpha\left(\pi_{\mathrm{yz}}^{*}\right) \beta\left(\pi_{\mathrm{OO}}^{*}\right)$ & 2.03 & 2.41 & 1.38 & 1.73 & 1.80 \\
\hline
\end{tabular}

Table S4.1.2. Spin and charge distributions and expectation value $<\mathrm{S}^{2}>$.

\begin{tabular}{|c|c|c|c|c|c|c|c|c|}
\hline & $\boldsymbol{F e}$ & $\mathrm{O}_{2}$ & $\mathrm{H}_{2} \mathrm{O}$ & Heme & $\boldsymbol{S H}$ & $\mathrm{H}_{4} \boldsymbol{B}$ & Arg $_{\text {substrate }}$ & $<$ S2> \\
\hline Spin & 1.14 & -1.01 & 0.00 & -0.10 & -0.02 & 0.00 & -0.01 & \multirow{2}{*}{1.03} \\
\cline { 1 - 8 } Charge & 0.18 & -0.40 & 0.00 & -1.28 & -0.46 & 0.57 & 0.95 & \\
\hline
\end{tabular}

Table S4.1.3. Relative energies ( $\mathrm{kcal} / \mathrm{mol})$.

\begin{tabular}{|c|c|c|c|c|}
\hline & \multicolumn{2}{|c|}{$Q M$ part of $Q M / M M$ energy } & \multicolumn{2}{|c|}{ Total QM/MM energy } \\
\hline & B1 & B2 & B1 & B2 \\
\hline Singlet & 0.00 & 0.00 & 0.00 & 0.00 \\
\hline
\end{tabular}




\section{Product}

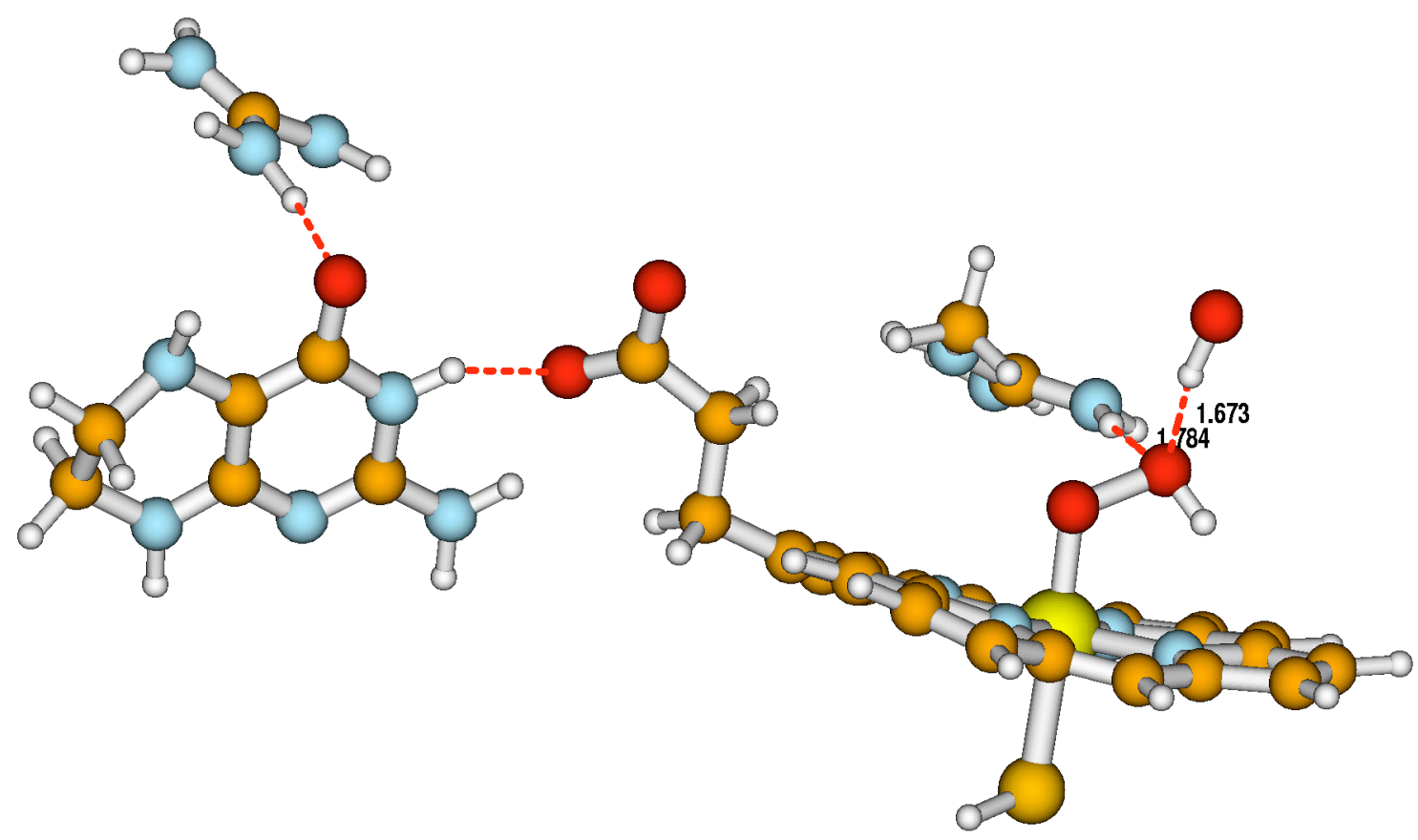

Table S4.2.1. Geometries (Å).

\begin{tabular}{|c|c|c|c|c|c|}
\hline State & $\boldsymbol{F e}-\boldsymbol{O}_{\text {inner }}$ & $\boldsymbol{F e}-\boldsymbol{S}$ & $\boldsymbol{O}_{\text {inner }}-\boldsymbol{O}_{\text {outer }}$ & ArgH $-\boldsymbol{O}_{\text {outer }}$ & $\boldsymbol{O}_{\text {outer }}-\boldsymbol{O H} \boldsymbol{O}$ \\
\hline$\alpha\left(\pi_{\mathrm{yz}}^{*}\right) \beta\left(\pi^{*} \mathrm{OH}\right)$ & 1.92 & 2.44 & 1.55 & 1.78 & 1.67 \\
\hline
\end{tabular}

Table S4.2.2. Spin and charge distributions and expectation value $<\mathrm{S}^{2}>$.

\begin{tabular}{|c|c|c|c|c|c|c|c|c|}
\hline & $\boldsymbol{F e}$ & $\boldsymbol{O O H}$ & $\bullet \boldsymbol{O H}$ & Heme & $\boldsymbol{S H}$ & $\boldsymbol{H}_{4} \boldsymbol{B}$ & Arg $_{\text {substrate }}$ & $<\boldsymbol{S} 2>$ \\
\hline Spin & 1.06 & 0.03 & -0.99 & -0.08 & -0.02 & 0.00 & 0.00 & 1.05 \\
\cline { 1 - 6 } Charge & 0.27 & -0.37 & 0.02 & -1.42 & -0.53 & 0.57 & 1.02 & \\
\hline
\end{tabular}

Table S4.2.3. Energies (relative to Table S4.1.3) ( kcal $/ \mathrm{mol})$.

\begin{tabular}{|c|c|c|c|c|}
\hline & \multicolumn{2}{|c|}{ QM part of QM/MM energy } & \multicolumn{2}{c|}{ Total QM/MM energy } \\
\hline & B1 & B2 & B1 & B2 \\
\hline Singlet & 35.11 & 43.01 & 32.80 & 40.71 \\
\hline
\end{tabular}




\section{Reaction 5}

Total QM charge: +1

No. of QM atoms: 97

\section{Reactant}

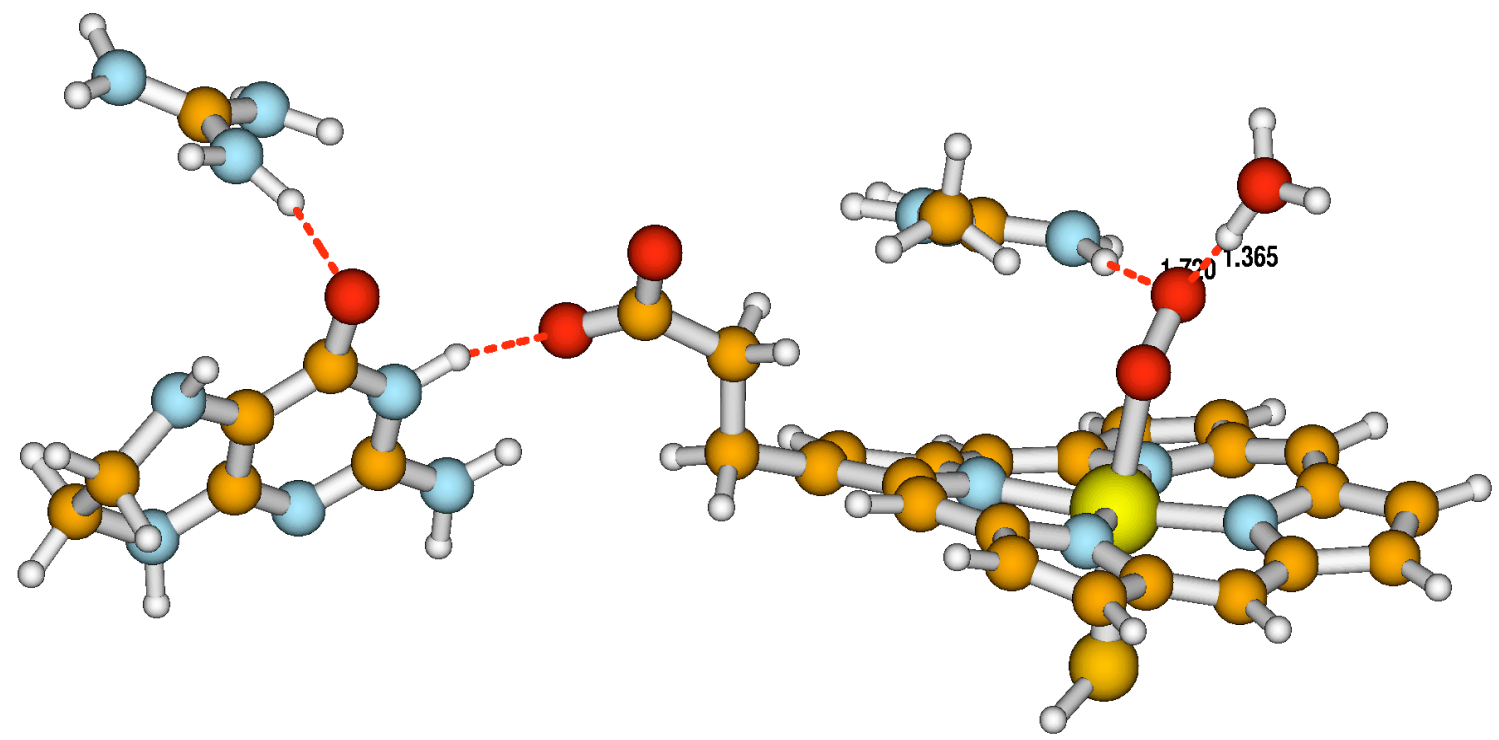

Table S5.1.1. Geometries $(\AA)$.

\begin{tabular}{|c|c|c|c|c|c|}
\hline State & $\boldsymbol{F e}-\boldsymbol{O}_{\text {inner }}$ & $\boldsymbol{F e}-\boldsymbol{S}$ & $\boldsymbol{O}_{\text {inner }}-\boldsymbol{O}_{\text {outer }}$ & ArgH $-\boldsymbol{O}_{\text {outer }}$ & $\boldsymbol{O}_{\text {outer }}-\boldsymbol{H}_{3} \boldsymbol{O}^{+}$ \\
\hline$\alpha\left(\pi_{\mathrm{yz}}^{*}\right) \beta\left(\pi_{\mathrm{OO}}^{*}\right)$ & 2.21 & 2.37 & 1.40 & 1.72 & 1.37 \\
\hline
\end{tabular}

Table S5.1.2. Spin and charge distributions and expectation value $<\mathrm{S}^{2}>$.

\begin{tabular}{|c|c|c|c|c|c|c|c|c|}
\hline & $\boldsymbol{F e}$ & $\boldsymbol{O}_{2}$ & $\boldsymbol{H}_{3} \boldsymbol{O}^{+}$ & Heme & $\boldsymbol{S H}$ & $\boldsymbol{H}_{4} \boldsymbol{B}$ & Arg $_{\text {substrate }}$ & $<$ S2> \\
\hline Spin & 1.22 & -0.94 & 0.01 & -0.19 & -0.10 & 0.00 & 0.00 & \multirow{2}{*}{1.12} \\
\cline { 1 - 8 } Charge & 0.06 & -0.46 & 0.83 & -1.14 & -0.27 & 0.06 & 0.97 & \\
\hline
\end{tabular}

Table S5.1.3. Energies (relative to Table S5.2.3) ( kcal $/ \mathrm{mol})$.

\begin{tabular}{|c|c|c|c|c|}
\hline & \multicolumn{2}{|c|}{ QM part of QM/MM energy } & \multicolumn{2}{c|}{ Total QM/MM energy } \\
\hline & B1 & B2 & B1 & B2 \\
\hline Singlet & 19.19 & 25.34 & 27.66 & 33.76 \\
\hline
\end{tabular}




\section{Intermediate}

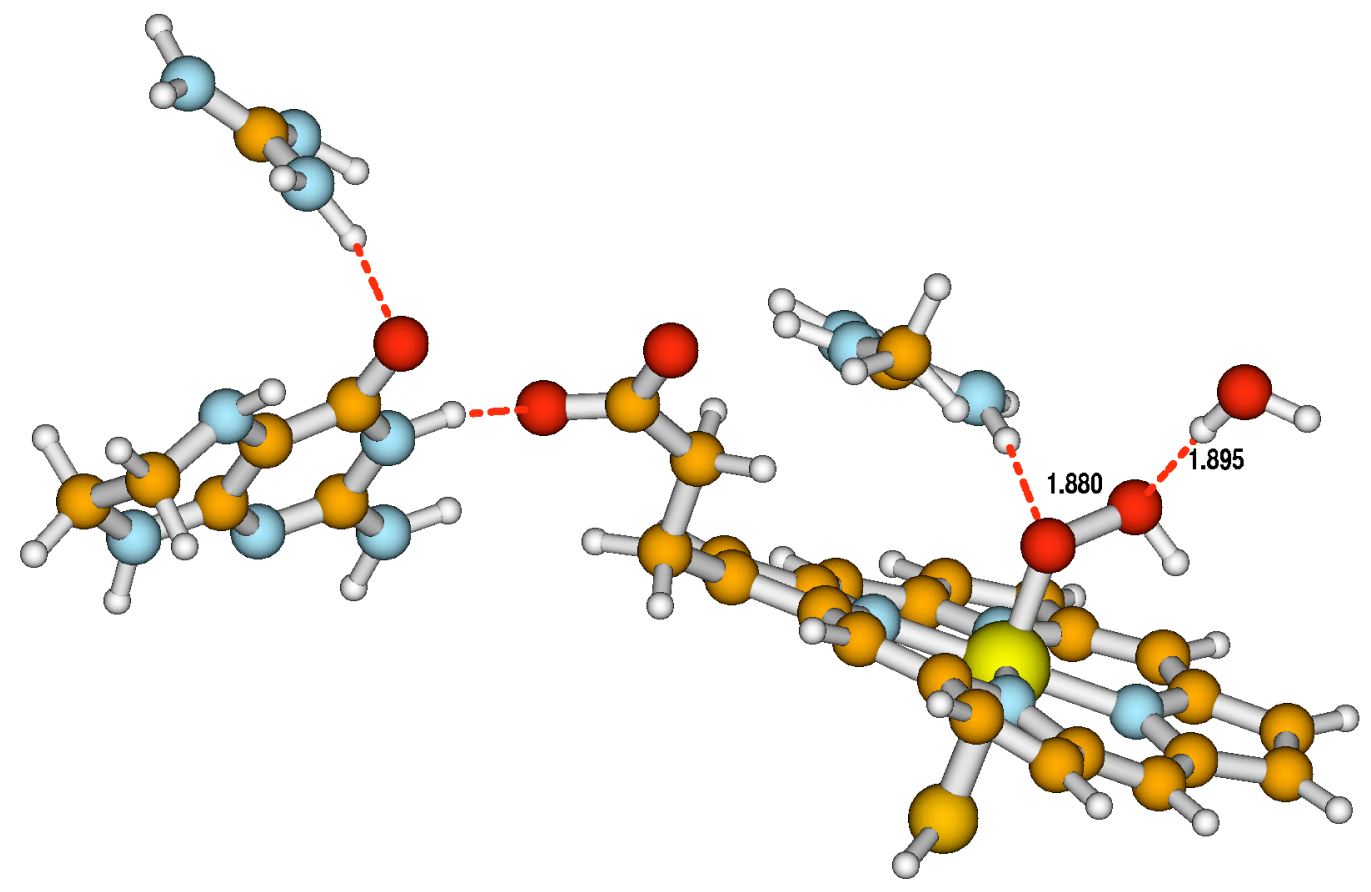

Table S5.2.1. Geometries ( $\AA$ ).

\begin{tabular}{|c|c|c|c|c|c|}
\hline State & $\boldsymbol{F e}-\boldsymbol{O}_{\text {inner }}$ & $\boldsymbol{F e}-\boldsymbol{S}$ & $\boldsymbol{O}_{\text {inner }}-\boldsymbol{O}_{\text {outer }}$ & ArgH $-\boldsymbol{O}_{\text {inner }}$ & $\boldsymbol{O}_{\text {outer }}-\boldsymbol{H}_{2} \boldsymbol{O}$ \\
\hline$\alpha\left(\pi_{\mathrm{yz}}^{*}\right) \beta\left(\mathrm{a}_{2 \mathrm{u}}\right)$ & 1.92 & 2.46 & 1.53 & 1.88 & 1.90 \\
\hline
\end{tabular}

Table S5.2.2. Spin and charge distributions and expectation value $<\mathrm{S}^{2}>$.

\begin{tabular}{|c|c|c|c|c|c|c|c|c|}
\hline & $F e$ & $O O H$ & $\mathrm{H}_{2} \mathrm{O}$ & Heme & $S H$ & $\boldsymbol{H}_{4} B$ & $\operatorname{Arg}_{\text {substrate }}$ & $<\boldsymbol{S} 2>$ \\
\hline Spin & 1.14 & -0.01 & 0.00 & -0.98 & -0.15 & 0.00 & 0.00 & \multirow{2}{*}{1.11} \\
\hline Charge & 0.11 & -0.21 & 0.03 & -0.59 & -0.34 & 0.06 & 1.00 & \\
\hline
\end{tabular}

Table S5.2.3. Relative energies ( $\mathrm{kcal} / \mathrm{mol})$.

\begin{tabular}{|c|c|c|c|c|}
\hline & \multicolumn{2}{|c|}{$Q M$ part of $Q M / M M$ energy } & \multicolumn{2}{|c|}{ Total QM/MM energy } \\
\hline & B1 & B2 & B1 & B2 \\
\hline Singlet & 0.00 & 0.00 & 0.00 & 0.00 \\
\hline
\end{tabular}




\section{Product}

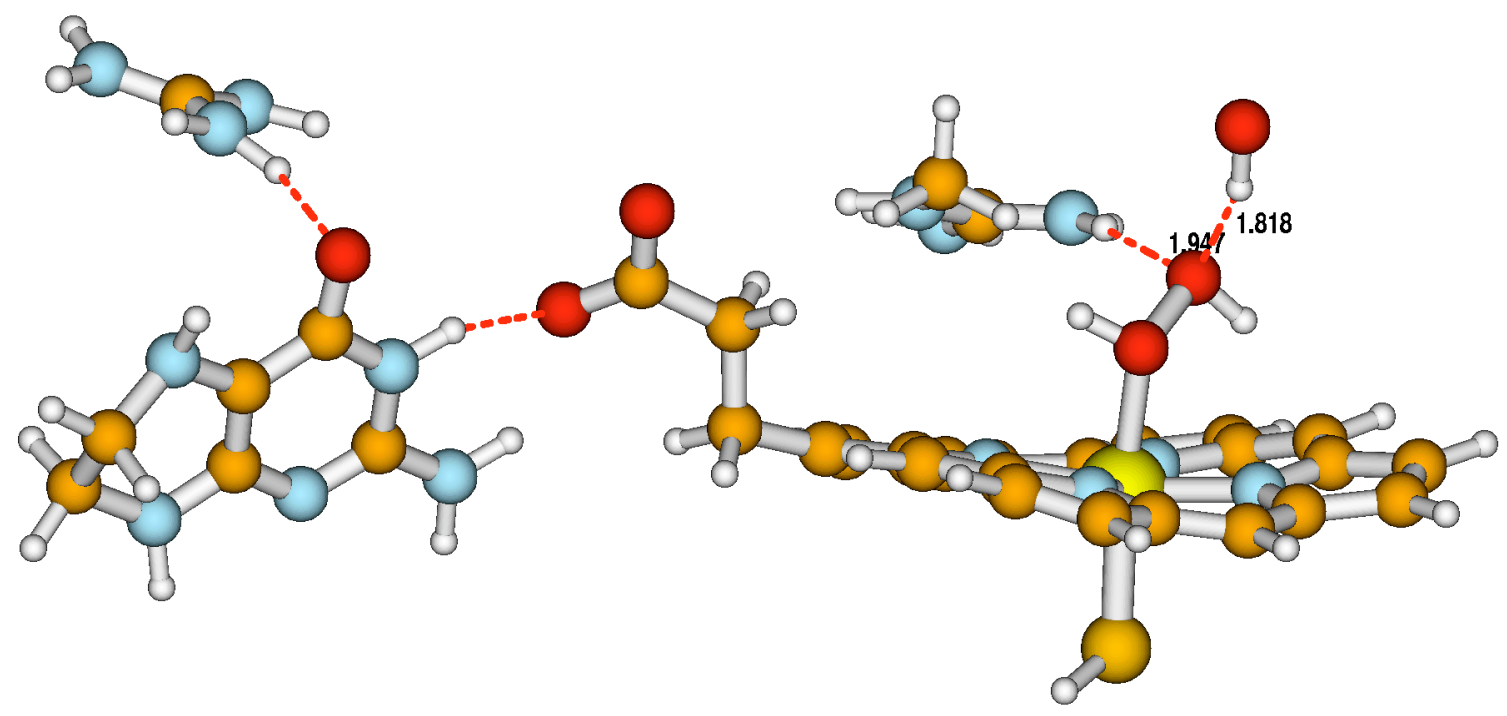

Table S5.3.1. Geometries $(\AA)$.

\begin{tabular}{|c|c|c|c|c|c|}
\hline State & $\boldsymbol{F e}-\boldsymbol{O}_{\text {inner }}$ & $\boldsymbol{F e}-\boldsymbol{S}$ & $\boldsymbol{O}_{\text {inner }}-\boldsymbol{O}_{\text {outer }}$ & ArgH $-\boldsymbol{O}_{\text {outer }}$ & $\boldsymbol{O}_{\text {outer }}-\boldsymbol{O H} \boldsymbol{O}$ \\
\hline$\alpha\left(\pi_{\mathrm{yz}}^{*}\right) \beta\left(\pi^{*} \mathrm{OH}\right)$ & 2.16 & 2.32 & 1.57 & 1.95 & 1.82 \\
\hline
\end{tabular}

Table S5.3.2. Spin and charge distributions and expectation value $<\mathrm{S}^{2}>$.

\begin{tabular}{|c|c|c|c|c|c|c|c|c|}
\hline & $\boldsymbol{F e}$ & $\mathrm{H}_{2} \boldsymbol{O}_{2}$ & $\bullet \boldsymbol{O H}$ & Heme & $\boldsymbol{S H}$ & $\boldsymbol{H}_{\mathbf{4}} \boldsymbol{B}$ & Arg $_{\text {substrate }}$ & $<\boldsymbol{S} 2>$ \\
\hline Spin & 1.14 & -0.01 & -0.99 & -0.09 & -0.05 & 0.00 & 0.00 & 1.10 \\
\cline { 1 - 6 } Charge & -0.02 & 0.17 & 0.03 & -1.05 & -0.18 & 0.07 & 1.03 & \\
\hline
\end{tabular}

Table S5.3.3. Energies (relative to Table S5.2.3) (kcal/mol).

\begin{tabular}{|c|c|c|c|c|}
\hline & \multicolumn{2}{|c|}{ QM part of QM/MM energy } & \multicolumn{2}{c|}{ Total QM/MM energy } \\
\hline & B1 & B2 & B1 & B2 \\
\hline Singlet & 52.88 & 55.84 & 50.46 & 53.39 \\
\hline
\end{tabular}




\section{Reaction 6}

Total QM charge: +2

No. of QM atoms: 98

\section{Reactant}

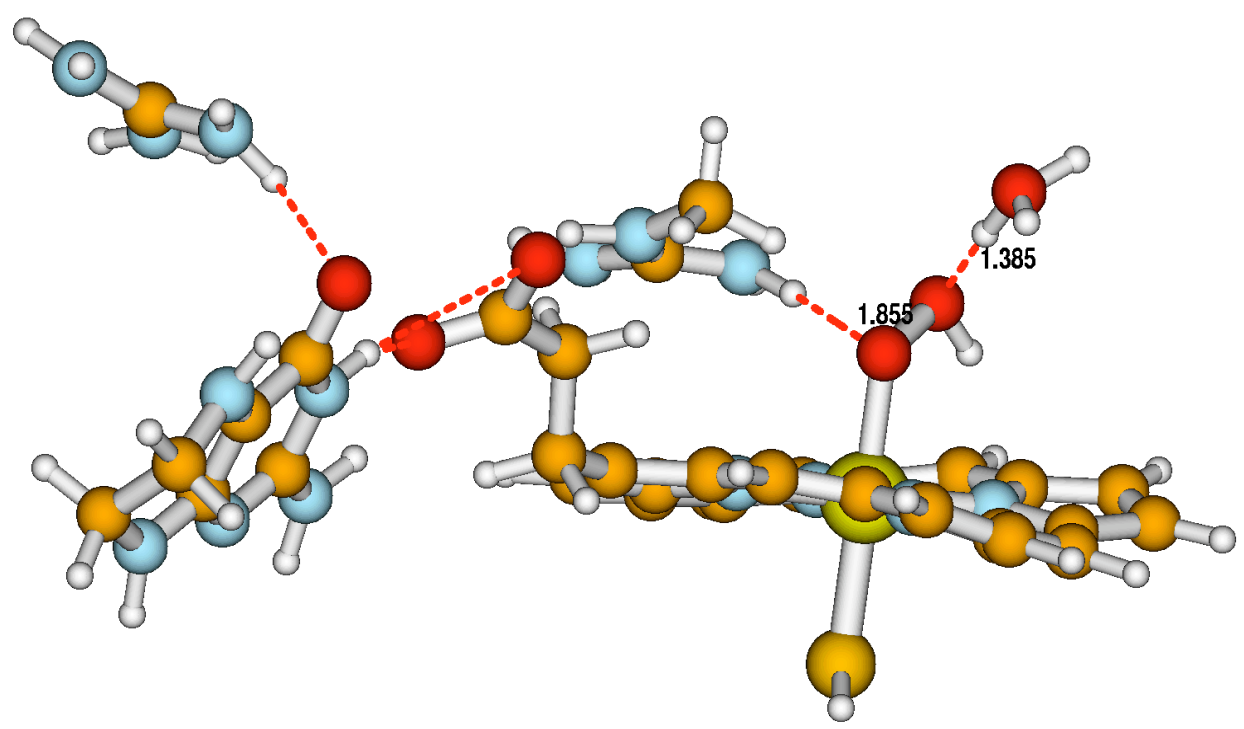

Table S6.1.1. Geometries (Å).

\begin{tabular}{|c|c|c|c|c|c|}
\hline State & $\boldsymbol{F e}-\boldsymbol{O}_{\text {inner }}$ & $\boldsymbol{F e}-\boldsymbol{S}$ & $\boldsymbol{O}_{\text {inner }}-\boldsymbol{O}_{\text {outer }}$ & ArgH $-\boldsymbol{O}_{\text {inner }}$ & $\boldsymbol{O}_{\text {outer }}-\boldsymbol{H}_{3} \boldsymbol{O}^{+}$ \\
\hline$\alpha\left(\pi_{\mathrm{yz}}^{*}\right) \beta\left(\mathrm{a}_{2 \mathrm{u}}+\mathrm{H}_{4} \mathrm{~B}\right)$ & 2.08 & 2.41 & 1.56 & 1.85 & 1.38 \\
\hline
\end{tabular}

Table S6.1.2. Spin and charge distributions and expectation value $<\mathrm{S}^{2}>$.

\begin{tabular}{|c|c|c|c|c|c|c|c|c|}
\hline & $\boldsymbol{F e}$ & $\boldsymbol{O O H}$ & $\mathrm{H}_{3} \boldsymbol{O}^{+}$ & Heme & $\boldsymbol{S H}$ & $\mathrm{H}_{4} \mathrm{~B}$ & Arg $_{\text {substrate }}$ & $<$ S2> \\
\hline Spin & 1.16 & -0.01 & 0.00 & -0.47 & -0.09 & -0.59 & 0.00 & 1.10 \\
\cline { 1 - 7 } Charge & 0.12 & -0.34 & 0.87 & -0.90 & -0.29 & 0.57 & 0.99 & \\
\hline
\end{tabular}

Table S6.1.3. Energies (relative to Table S5.2.3) (kcal/mol).

\begin{tabular}{|c|c|c|c|c|}
\hline & \multicolumn{2}{|c|}{ QM part of $\mathbf{Q M} / \mathbf{M M}$ energy } & \multicolumn{2}{c|}{ Total QM/MM energy } \\
\hline & B1 & B2 & B1 & B2 \\
\hline Singlet & 12.00 & 25.78 & 20.70 & 34.40 \\
\hline
\end{tabular}




\section{Product}

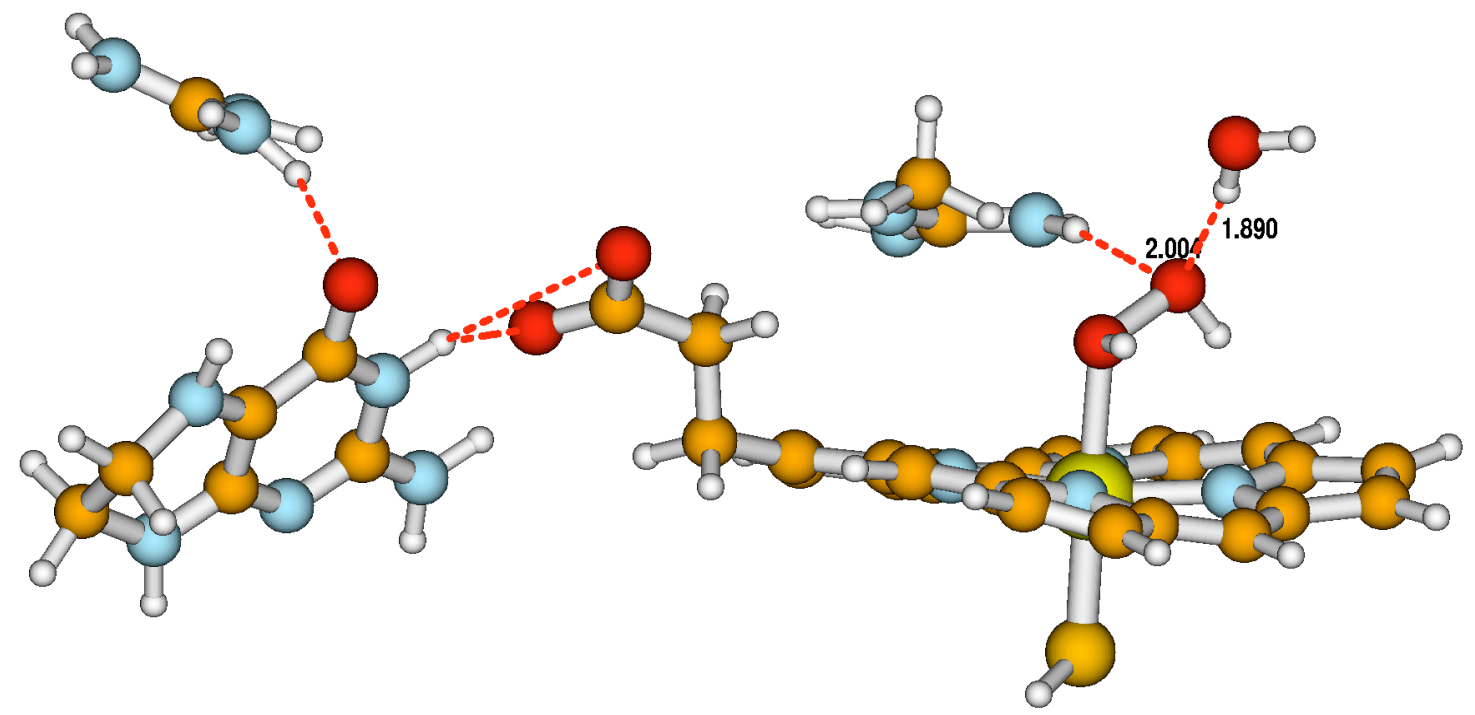

Table S6.2.1. Geometries (A)).

\begin{tabular}{|c|c|c|c|c|c|}
\hline State & $\boldsymbol{F e}-\boldsymbol{O}_{\text {inner }}$ & $\boldsymbol{F e}-\boldsymbol{S}$ & $\boldsymbol{O}_{\text {inner }}-\boldsymbol{O}_{\text {outer }}$ & ArgH $-\boldsymbol{O}_{\text {inner }}$ & $\boldsymbol{O}_{\text {outer }}-\boldsymbol{H}_{2} \boldsymbol{O}$ \\
\hline$\alpha\left(\pi^{*}{ }_{\mathrm{yz}}\right) \beta\left(\mathrm{H}_{4} \mathrm{~B}\right)$ & 2.28 & 2.33 & 1.53 & 2.00 & 1.89 \\
\hline
\end{tabular}

Table S6.2.2. Spin and charge distributions and expectation value $<\mathrm{S}^{2}>$.

\begin{tabular}{|c|c|c|c|c|c|c|c|c|}
\hline & $F e$ & $\mathrm{H}_{2} \mathrm{O}_{2}$ & $\mathrm{H}_{2} \mathrm{O}$ & Heme & $\boldsymbol{S H}$ & $\mathrm{H}_{4} \mathrm{~B}$ & Arg $_{\text {substrate }}$ & $<$ S2> \\
\hline Spin & 1.18 & -0.01 & 0.00 & -0.27 & -0.08 & -0.82 & 0.00 & \multirow{2}{*}{1.13} \\
\cline { 1 - 7 } Charge & 0.00 & 0.24 & 0.03 & -0.91 & -0.14 & 0.78 & 1.01 & \\
\hline
\end{tabular}

Table S6.2.3. Relative energies ( $\mathrm{kcal} / \mathrm{mol})$.

\begin{tabular}{|c|c|c|c|cc|c|}
\hline & \multicolumn{3}{|c|}{ QM part of QM/MM energy } & \multicolumn{3}{c|}{ Total QM/MM energy } \\
\hline & B1 & \multicolumn{2}{|c|}{ B2 } & B1 & B2 \\
\hline Singlet & \multicolumn{2}{|c|}{$\mathbf{0 . 0 0}$} & 0.00 & & 0.00 & 0.00 \\
\hline
\end{tabular}




\section{References}

(S1) Chemshell 2.05b4, incl DL-POLY, Sherwood, P.; de Vries, A. H.; Guest, M. F.; Schreckenbach, G.; Catlow, C. R. A.; French, S. A.; Sokol, A. A.; Bromley, S. T.; Thiel, W.; Turner, A. J.; Billeter, S.; Terstegen, F.; Thiel, S.; Kendrick, J.; Rogers, S. C.; Casci, J.; Watson, M.; King, F.; Karlsen, E.; Sjøvoll, M.; Fahmi, A.; Schäfer, A.; Lennartz, C. J. Mol. Struct. Theochem. 2003, 632, 128.

(S2) Turbomol 5.5, Ahlrichs, R.; Bär, M.; Häser, M.; Horn, H.; Kölmel, C. Chem. Phys. Lett. 1989, 162, 165-169.

(S3) Jaguar 5.5, Schrodinger, LLC, Portland, Oregon, 2003

(S4) (a) Lee, C.; Yang, W.; Parr, R. G. Phys. ReV. B 1988, 37, 785-789. (b) Becke, A. D. Phys. ReV. A 1988, 38, 3098-3100. (c) Becke, A. D. J. Chem. Phys. 1993, 98, 1372-1377. (d) Becke, A. D. J. Chem. Phys. 1993, 98, 5648-5652.

(S5) Neese, F. ORCA, Version 2.4, Revision 10, Max-Planck-Institut für Bioanorganische Chemie, Mülheim a. d. Ruhr, Germany, 2004

(S6) Neese, F. Inorg. Chim. Acta 2002, 337, 181.

(S7) Schäfer, A.; Horn, H.; Ahlrichs, R. J. Chem. Phys. 1992, 97, 2571.

(S8) Hui Li, Andrew D. Robertson, and Jan H. Proteins, 2005, 61, 704-721 


\section{Coordinates}

QM region coordinates are supplied here in xyz-format.

$* * * * * * * * * * * * * * * * * * * * * * * * * * * * * * * * * *$
REACTION 1 SINGLET
REACTANT
TOTAL QM CHARGE: 0
$* * * * * * * * * * * * * * * * * * * * * * * * * * * * * * * * * * *$

93

S $\quad 6.134961-1.031158-5.640273$

O $5.8695430 .597688-1.536191$

O $6.9086861 .167213-0.842802$

FE $6.019184-0.001166-3.458400$

$\mathrm{N} \quad 4.8430001 .499131-4.125607$

N $7.6896551 .050610-3.994746$

N $7.190011-1.431077-2.669008$

N $\quad 4.360269-0.985916-2.867151$

C $3.4717871 .628307-3.971802$

C $3.0091502 .866419-4.587276$

C $4.1155773 .473934-5.140461$

C $5.2512222 .610416-4.849393$

C $7.7003732 .204375-4.755755$

C $9.0715792 .655925-4.963570$

C $9.9042531 .770691-4.324846$

C $9.0194940 .752282-3.713249$

C $8.549387-1.352476-2.444022$

C $9.004016-2.537793-1.708040$

C $7.923664-3.360063-1.546810$

C $6.777603-2.643482-2.146025$

C $4.324833-2.285983-2.400947$

C $2.946577-2.668313-2.091276$

C $2.173533-1.562834-2.313691$

C $3.052028-0.527626-2.847508$

C $2.6452560 .697598-3.361987$

H $\quad 1.5854470 .922299-3.350650$

C $6.5694952 .895910-5.178252$

H $\quad 6.7363153 .813704-5.732519$

C $9.376941-0.339768-2.923302$

H $\quad 10.416300-0.434414-2.657445$

C $5.447989-3.059535-2.126849$

H $5.243286-4.052184-1.739320$

C $1.6034553 .404377-4.434984$

H $\quad 0.8582612 .629304-4.651956$

H $\quad 1.4338194 .211978-5.156172$

C $1.4232153 .955214-3.004952$

H $\quad 2.3194904 .556465-2.789960$

H $1.4229743 .131912-2.282542$

C $\quad 0.2288364 .842398-2.674647$

O $\quad-0.0630344 .944138-1.424190$

O $\quad-0.3794065 .513326-3.602075$

N $\quad-3.1774219 .969880-3.033748$

H $\quad-2.6724539 .105328-2.813889$

C $-4.53683910 .001494-2.812361$

$\mathrm{N} \quad-5.21693311 .161651-2.736566$

$\mathrm{H} \quad-6.22870711 .158714-2.869055$

H $\quad-4.80607412 .112502-2.823654$

N $\quad-5.1567178 .852679-2.586888$

H $-4.7733837 .894096-2.758843$

H $\quad-6.1516578 .882951-2.302550$

$\mathrm{N} \quad-1.3460005 .227343-6.280926$

H $\quad-1.1306204 .961002-7.233208$

H $\quad-0.6854725 .092415-5.522030$

C $\quad-2.6335205 .558811-5.993075$

$\mathrm{N} \quad-3.5239485 .688128-6.963918$

N $\quad-2.9756965 .771066-4.682688$

H $\quad-2.2533835 .685590-3.964433$

C $-4.2861196 .059205-4.286501$

O $\quad-4.5152766 .279551-3.023836$

C $\quad-5.2484186 .130938-5.308465$
C $\quad-4.8224865 .935950-6.635016$

N $\quad-6.6147036 .371700-5.023430$

$\mathrm{H} \quad-6.8681896 .138307-4.068798$

$\mathrm{N} \quad-5.7158245 .995581-7.658770$

H $\quad-5.3871075 .756704-8.587091$

C $\quad-7.5823555 .949142-6.070988$

H $\quad-7.6383214 .853059-6.131210$

C $\quad-7.0819616 .470260-7.435766$

H $\quad-7.7079146 .088348-8.246837$

H $\quad-7.1170757 .566926-7.462513$

C $4.6998323 .824754-0.661580$

H $\quad 3.6679393 .676195-0.992883$

H $5.1526772 .833244-0.623360$

N $5.3691654 .665020-1.666165$

H $4.8010685 .434960-2.065097$

C $6.6438464 .619084-2.081023$

$\mathrm{N} \quad 7.5196103 .668483-1.708502$

H $7.2389872 .729037-1.389706$

H $\quad 8.4422853 .725911-2.119630$

N $7.0717775 .601226-2.901633$

H $8.0571785 .626983-3.144040$

H $6.4786236 .440367-3.057266$

h $4.890782-1.556886-5.923071$

h $4.1933104 .449797-5.619747$

h $9.2987003 .530843-5.572669$

h $\quad 10.9891601 .872202-4.352043$

h $10.024503-2.669594-1.348456$

h $7.812351-4.333412-1.069036$

h $2.653689-3.659788-1.745935$

h $1.112094-1.416236-2.113881$

h $-2.65754210 .642005-3.560953$

h $-8.5742476 .334240-5.834521$

h $\quad 4.7068434 .2650820 .335480$

REACTION 1 SINGLET PRODUCT TOTAL QM CHARGE: 0

$* * * * * * * * * * * * * * * * * * * * * * * * * * * * * * * * * *$

93

S $\quad 6.121197-1.054566-5.741738$

O $6.1136430 .806488-1.930447$

O 7.7308941 .5621480 .128106

FE $6.0658230 .075685-3.418179$

$\mathrm{N} \quad 4.8902031 .530452-4.211930$

N $7.7394551 .040451-4.095285$

N $7.211878-1.441110-2.742664$

N $\quad 4.398919-0.955537-2.917332$

C $3.5188971 .661318-4.068733$

C $3.0662142 .940168-4.604896$

C $\quad 4.1885143 .589149-5.068754$

C $\quad 5.3109082 .698875-4.816530$

C $7.7656312 .268083-4.725394$

C $9.1393882 .707268-4.929663$

C $9.9654581 .731652-4.434059$

C $9.0687420 .690620-3.884179$

C $8.576953-1.398618-2.572844$

C $9.032665-2.562336-1.807670$

C $7.942098-3.356488-1.586141$

C $6.797226-2.632886-2.170259$

C $4.344562-2.243545-2.419721$

C $2.966676-2.613542-2.118661$

C $2.199602-1.512248-2.384315$

C $3.089043-0.496971-2.927568$

C $2.6943730 .720988-3.473368$

H $\quad 1.6352430 .949263-3.462941$

C $6.6413873 .009373-5.071653$
H $\quad 6.8188933 .966745-5.546425$

C $9.413874-0.429759-3.129216$

H $\quad 10.460471-0.572429-2.920492$

C $\quad 5.462912-3.019235-2.119603$

H $\quad 5.247769-3.998844-1.706212$

C $1.6563433 .467402-4.452387$

H $\quad 0.9166742 .704041-4.723500$

$\mathrm{H} \quad 1.5041334 .305464-5.141706$

C $1.4451593 .958246-3.003865$

H $\quad 2.3459874 .524063-2.727679$

H $\quad 1.3978503 .109114-2.313401$

C $0.2599664 .862016-2.681556$

O $\quad-0.0301184 .979524-1.432645$

O $\quad-0.3504755 .520564-3.616443$

$\mathrm{N} \quad-3.1758819 .968201-3.036606$

$\mathrm{H} \quad-2.6688159 .105549-2.813976$

C $\quad-4.53473110 .002281-2.814269$

N $\quad-5.21494411 .162220-2.740350$

H $\quad-6.22699511 .158080-2.870968$

H $\quad-4.80718212 .115321-2.825385$

N $\quad-5.1553018 .853438-2.588540$

H $\quad-4.7710347 .895664-2.759422$

H $\quad-6.1490108 .883301-2.300788$

N $\quad-1.3447805 .226278-6.280117$

H $\quad-1.1269774 .969138-7.234197$

H $\quad-0.6792765 .107168-5.522760$

C $\quad-2.6318545 .558984-5.993425$

$\mathrm{N} \quad-3.5207375 .687376-6.965696$

N $\quad-2.9750085 .772926-4.683765$

H $\quad-2.2516205 .694279-3.966827$

C $-4.2869786 .058494-4.288102$

O $\quad-4.5177186 .277161-3.026069$

C $\quad-5.2475176 .130276-5.311859$

C $\quad-4.8195005 .936212-6.637708$

N $\quad-6.6144296 .369303-5.028662$

H $\quad-6.8676796 .134586-4.074292$

$\mathrm{N} \quad-5.7113055 .995661-7.662268$

H $\quad-5.3798005 .759012-8.590022$

C $\quad-7.5802895 .949882-6.078222$

H $\quad-7.6360984 .853800-6.142402$

C $\quad-7.0765486 .473492-7.440678$

$\mathrm{H} \quad-7.7008446 .097047-8.255306$

H $\quad-7.1055377 .570550-7.464139$

C $4.4650983 .916883-0.406630$

H $\quad 3.4307753 .784161-0.745507$

H $4.9120352 .917143-0.352250$

N $5.1670594 .701245-1.426290$

H $4.6375385 .396849-1.974022$

C $\quad 6.5226624 .685394-1.613201$

$\mathrm{N} 7.3447164 .022265-0.828572$

H $7.6943122 .533098-0.096848$

H $7.2508301 .116064-0.607997$

N $6.9967355 .428183-2.677960$

$\mathrm{H} \quad 8.0128425 .462360-2.770031$

H $6.4455576 .263489-2.954220$

h $4.869245-1.575190-5.998473$

h $4.2924534 .606492-5.445950$

h $9.3627703 .666723-5.396153$

h $11.0517421 .802362-4.489325$

h $10.053101-2.676624-1.441999$

h $7.829403-4.313301-1.076362$

h $2.667716-3.594257-1.748678$

h $1.136152-1.360910-2.199281$

h $-2.65443710 .641162-3.561193$

h $\quad-8.5730786 .333344-5.842864$

h 4.4552884 .3518430 .592760 
REACTION 1 TRIPLET REACTANT

TOTAL QM CHARGE: 0

*************************************

93

S $\quad 6.134116-1.027119-5.650038$

O $5.8761530 .570720-1.491620$

O $6.8878551 .211172-0.812635$

FE $6.021511-0.013911-3.472694$

N $\quad 4.8476001 .495682-4.126798$

$\mathrm{N} \quad 7.6905861 .042901-4.005235$

N $7.188656-1.433329-2.672240$

N $4.363754-0.988634-2.870408$

C $3.4766391 .627974-3.969050$

C $3.0135272 .865886-4.583868$

C $4.1193853 .471652-5.140326$

C $5.2542772 .606209-4.853522$

C $7.7018672 .197236-4.765919$

C $\quad 9.0726662 .650809-4.970121$

C $9.9052951 .765990-4.330638$

C $9.0205190 .746519-3.721146$

C $8.549401-1.355236-2.448670$

C $9.003102-2.538213-1.709904$

C $7.923070-3.361113-1.548631$

C $6.777076-2.645929-2.147976$

C $4.326027-2.288762-2.404031$

C 2.947919-2.668687-2.092559

C $2.176177-1.562090-2.313841$

C $3.055303-0.528209-2.847867$

C $2.6497120 .698446-3.358420$

H $\quad 1.5904850 .925318-3.343120$

C $6.5714662 .889883-5.187258$

H $\quad 6.7382323 .807835-5.741239$

C $9.377501-0.344614-2.929818$

H $\quad 10.416288-0.437487-2.661507$

C $5.448007-3.063185-2.129518$

H $5.242728-4.055181-1.740803$

C $1.6074513 .403426-4.432493$

H $\quad 0.8628722 .625978-4.643044$

H $\quad 1.4353204 .205393-5.159440$

C $1.4284993 .963191-3.005926$

H $2.3208064 .573103-2.798476$

H $\quad 1.4392243 .144601-2.278123$

C $\quad 0.2278154 .842278-2.676092$

O $\quad-0.0657664 .941983-1.425870$

O $\quad-0.3835765 .510010-3.603739$

N $\quad-3.1780209 .969171-3.034291$

H $\quad-2.6723029 .105408-2.813302$

C $\quad-4.53688810 .002500-2.810917$

$\mathrm{N} \quad-5.21667611 .162887-2.734761$

H $\quad-6.22869611 .158622-2.864470$

H $\quad-4.80727412 .114784-2.821644$

N $\quad-5.1575858 .853925-2.587954$

$\mathrm{H} \quad-4.7751147 .895150-2.761876$

H $\quad-6.1519208 .884428-2.302407$

N $\quad-1.3495765 .232190-6.279009$

H $\quad-1.1317614 .967787-7.231037$

H $\quad-0.6902555 .099103-5.518464$

C $-2.6386165 .559807-5.993625$

N $\quad-3.5266145 .691465-6.966458$

$\mathrm{N} \quad-2.9826735 .770371-4.683649$

H $\quad-2.2613255 .683601-3.964742$

C $-4.2924566 .062772-4.289076$

O $\quad-4.5233996 .281082-3.026542$

C $\quad-5.2527596 .134599-5.312361$

C $\quad-4.8254405 .939607-6.639058$

N $\quad-6.6188286 .381171-5.028812$

H $\quad-6.8712636 .139186-4.075810$

N $\quad-5.7174166 .002183-7.663574$

H $\quad-5.3888015 .760628-8.591125$

C $\quad-7.5846125 .953974-6.076827$
H $\quad-7.6386924 .857429-6.134772$

C $\quad-7.0844066 .474568-7.441658$

H $\quad-7.7099286 .092333-8.252849$

H $\quad-7.1204307 .571375-7.469173$

C $4.7011743 .826703-0.664702$

H $\quad 3.6682053 .681151-0.994268$

H $5.1532812 .834852-0.627602$

N $5.3704404 .667440-1.668984$

H $\quad 4.8024445 .437438-2.067324$

C $\quad 6.6448674 .620575-2.085968$

$\mathrm{N} \quad 7.5195383 .668724-1.717907$

H $\quad 7.2384042 .727353-1.394074$

H $8.4426603 .729684-2.127213$

N $7.0707685 .603488-2.908006$

$\mathrm{H} \quad 8.0563665 .630671-3.148912$

H $\quad 6.4780156 .443476-3.059158$

h $4.889691-1.554132-5.929339$

h $4.1961834 .447143-5.620520$

h $9.3000183 .526586-5.577897$

h $\quad 10.9901251 .868996-4.355124$

h $10.023281-2.668868-1.349028$

h $7.811857-4.334153-1.070204$

h $2.653673-3.659566-1.746660$

h $1.114705-1.415065-2.114525$

h $-2.65838710 .642025-3.560808$

h $\quad-8.5773666 .336826-5.840331$

h 4.7094334 .2654670 .333037

$* * * * * * * * * * * * * * * * * * * * * * * * * * * * * * * * * *$

REACTION 1 TRIPLET PRODUCT

TOTAL QM CHARGE: 0

*****************************************

93

S $6.120737-1.055324-5.742312$

O $6.1145000 .803699-1.931000$

O 7.7311991 .5641110 .130311

FE $6.0666560 .074147-3.418445$

N $4.8906191 .529174-4.210870$

N $7.7399241 .037622-4.097561$

N $7.213242-1.441827-2.743235$

N $4.399856-0.957249-2.918665$

C $3.5211631 .661522-4.067640$

C $3.0676892 .940028-4.603751$

C $4.1900213 .588913-5.068030$

C $5.3120882 .698524-4.816875$

C $7.7666622 .264641-4.725458$

C $9.1399682 .705395-4.929976$

C $9.9667651 .730064-4.435137$

C $9.0706960 .688239-3.885332$

C $8.576654-1.400089-2.572586$

C $9.032731-2.563537-1.807310$

C $7.942281-3.358072-1.585906$

C $6.797612-2.634706-2.169907$

C $4.344479-2.243820-2.421433$

C $2.966882-2.613589-2.119257$

C $2.199959-1.512060-2.383811$

C $3.088910-0.497195-2.927270$

C $2.6955280 .720536-3.471793$

H $1.6368680 .950544-3.460736$

C $6.6414993 .007199-5.071388$

H $\quad 6.8201353 .965618-5.543384$

C $9.414549-0.430301-3.129289$

H $\quad 10.460877-0.572987-2.919434$

C 5.464054-3.019944-2.120086

H $\quad 5.247737-3.999181-1.706107$

C $1.6576753 .467010-4.451780$

H $\quad 0.9180372 .702829-4.720555$

H $1.5041624 .303742-5.142460$

C $1.4465623 .959971-3.003907$

H $2.3463064 .527467-2.727232$

H $1.4030193 .111955-2.311574$
C $\quad 0.2594924 .861222-2.682225$

O $\quad-0.0327904 .977596-1.433871$

O $\quad-0.3507265 .518930-3.617905$

N $\quad-3.1757769 .968708-3.036884$

$\mathrm{H} \quad-2.6695759 .106040-2.812972$

C $\quad-4.53434010 .003383-2.813277$

$\mathrm{N} \quad-5.21435911 .163345-2.739216$

$\mathrm{H} \quad-6.22637211 .158274-2.869203$

H $\quad-4.80577012 .116005-2.824556$

N $\quad-5.1561028 .854985-2.589251$

H $\quad-4.7728217 .897813-2.762505$

H $\quad-6.1496328 .886253-2.301042$

$\mathrm{N} \quad-1.3442265 .228369-6.278164$

H $\quad-1.1258604 .971964-7.232247$

H $\quad-0.6800255 .111163-5.519190$

C $\quad-2.6322855 .559134-5.992978$

$\mathrm{N} \quad-3.5208035 .687662-6.965700$

$\mathrm{N} \quad-2.9763335 .772647-4.683507$

$\mathrm{H} \quad-2.2543245 .693838-3.965178$

C $-4.2881296 .059645-4.288933$

O $\quad-4.5205516 .276883-3.027096$

C $\quad-5.2482326 .129760-5.313258$

C $-4.8201025 .935513-6.638876$

$\mathrm{N} \quad-6.6148486 .372302-5.030328$

H $\quad-6.8684936 .136749-4.076065$

N $\quad-5.7113355 .998723-7.663958$

H $\quad-5.3813845 .762062-8.592263$

C $\quad-7.5803615 .950571-6.079671$

$\mathrm{H} \quad-7.6369174 .854315-6.141911$

C $\quad-7.0766176 .475411-7.441906$

H $\quad-7.7019606 .100051-8.256186$

H $\quad-7.1087667 .572598-7.463478$

C $4.4653823 .918236-0.406977$

H $3.4300023 .785751-0.743760$

H $4.9115312 .918054-0.351662$

N $5.1677214 .699705-1.427424$

H $4.6410205 .396377-1.975422$

C $\quad 6.5259464 .682011-1.610607$

N $7.3448474 .019388-0.827294$

H $7.6927802 .536856-0.098636$

H $7.2515341 .117486-0.604765$

N $6.9948625 .426377-2.680030$

H $8.0105395 .465036-2.770553$

H $6.4436066 .264454-2.946265$

h $4.868743-1.575819-5.999105$

h $4.2938804 .606575-5.444385$

h $9.3629223 .665612-5.395101$

h $11.0530481 .801222-4.489848$

h $10.053204-2.677222-1.441556$

h $7.829448-4.314737-1.075878$

h $2.667866-3.594176-1.748980$

h $1.136484-1.360648-2.198983$

h $-2.65503910 .642086-3.561638$

h $\quad-8.5734026 .333936-5.845218$

h 4.4558564 .3522340 .592834

\section{$* * * * * * * * * * * * * * * * * * * * * * * * * * * * * * * * * *$ REACTION 1 QUINTET \\ REACTANT \\ TOTAL QM CHARGE: 0 \\ ***************************************}

93

S $\quad 6.155131-1.153768-5.854076$

O $\quad 6.0738020 .573837-1.137997$

O $\quad 6.8671431 .405937-0.366007$

FE $6.0780590 .010916-3.508966$

N $4.9095051 .532292-4.141514$

N $7.7418841 .064998-4.030517$

N $7.229137-1.404594-2.677646$

N $4.424798-0.938398-2.852538$

C $3.5375401 .666147-3.981772$ 
$\begin{array}{llll}\text { C } & 3.0747372 .900825 & -4.598931 \\ \text { C } & 4.1813943 .506113-5.156907\end{array}$

C $5.3161992 .645895-4.867564$

C $7.7582032 .226298-4.785412$

C $9.1294842 .662652-4.997256$

C $9.9580431 .766664-4.365968$

C $9.0726390 .754310-3.755506$

C $8.593808-1.334196-2.466430$

C $9.045783-2.515190-1.726309$

C $7.963093-3.333046-1.553563$

C $6.817912-2.615045-2.142364$

C $4.376483-2.242962-2.386764$

C $2.997886-2.617140-2.090908$

C $2.230674-1.506756-2.315213$

C $3.113900-0.475532-2.840339$

C $2.7095600 .745774-3.361228$

H $1.6516240 .975822-3.344076$

C $6.6323432 .927583-5.201729$

H $\quad 6.8021623 .845301-5.753914$

C $9.426876-0.336673-2.963085$

H $\quad 10.467200-0.439651-2.706241$

C $5.489733-3.027897-2.116351$

H $\quad 5.279991-4.019810-1.731364$

C $1.6654273 .434825-4.453430$

H $\quad 0.9249002 .648415-4.642659$

H $\quad 1.4855624 .218232-5.198478$

C $1.4925474 .027285-3.038216$

H $2.3583394 .684466-2.871376$

H $\quad 1.5593833 .230628-2.290138$

C $\quad 0.2583414 .856968-2.702369$

O $-0.0432634 .935131-1.452735$

O $\quad-0.3706795 .513438-3.626793$

N $\quad-3.1790069 .967872-3.039408$

H $\quad-2.6727799 .104032-2.820575$

C $\quad-4.53731010 .001923-2.812973$

N $\quad-5.21692411 .162235-2.734243$

H $\quad-6.22916711 .157639-2.862578$

H $\quad-4.80827912 .114446-2.821989$

N $\quad-5.1579328 .853161-2.589916$

H $\quad-4.7753187 .894805-2.764489$

H $\quad-6.1517678 .883687-2.302497$

N $\quad-1.3582935 .234270-6.291878$

H $\quad-1.1443334 .971546-7.245523$

$\mathrm{H} \quad-0.6945555 .104880-5.534608$

C $-2.6457925 .562835-6.001597$

$\mathrm{N} \quad-3.5365245 .697985-6.971543$

$\mathrm{N} \quad-2.9858755 .769866-4.690007$

$\mathrm{H} \quad-2.2625245 .679929-3.973911$

C $-4.2947096 .062168-4.291251$

O $-4.5219436 .279112-3.027801$

C $\quad-5.2576376 .136793-5.312051$

C $-4.8344975 .945374-6.639935$

N $\quad-6.6231586 .381801-5.024431$

H $\quad-6.8723326 .140254-4.070317$

N $\quad-5.7288336 .010555-7.662165$

H $\quad-5.4010985 .772021-8.590800$

C $\quad-7.5901915 .950426-6.069346$

H $\quad-7.6381924 .853677-6.128611$

C $\quad-7.0977146 .475820-7.435160$

H $\quad-7.7241346 .091953-8.244928$

H $\quad-7.1387857 .572447-7.461347$

C $4.6365923 .856805-0.645848$

H $3.6000043 .750159-0.979557$

H $5.0611672 .852287-0.619482$

N $5.3352464 .690159-1.635336$

H $4.7890045 .472540-2.038579$

C $\quad 6.6116554 .605853-2.045159$

N $7.4597523 .649714-1.644478$

H $7.1813112 .763939-1.152124$

H $8.3784513 .659258-2.066032$

$\mathrm{N} \quad 7.0546935 .557536-2.899899$

H $8.0492505 .585191-3.105242$

H $6.4735266 .400666-3.072364$ h $\quad 4.869016-1.620044-6.035297$

h $4.2583334 .477497-5.645332$

h $\quad 9.3633743 .538745-5.602089$

h $11.0434991 .862414-4.392107$

h $10.066071-2.646938-1.366142$

h $7.853062-4.305973-1.074633$

h $2.697089-3.609412-1.754776$

h $1.165700-1.364642-2.131638$

h $\quad-2.65912610 .643018-3.562737$

h $\quad-8.5841246 .327277-5.828209$

h 4.6532704 .2820230 .357635

$* * * * * * * * * * * * * * * * * * * * * * * * * * * * * * * * * *$
REACTION 1 QUINTET
PRODUCT
TOTAL QM CHARGE: 0
$* * * * * * * * * * * * * * * * * * * * * * * * * * * * * * * * * *$

93

S $6.120213-1.051017-5.738355$

O $6.1150290 .802283-1.934945$

O 7.7302511 .5654930 .128411

FE $6.0655680 .068646-3.422650$

$\mathrm{N} \quad 4.8902191 .529237-4.210879$

N $7.7403371 .038067-4.096377$

N $7.212468-1.442900-2.743679$

N $4.400607-0.956713-2.917660$

C $3.5206921 .661430-4.067464$

C $3.0679182 .940016-4.603645$

C $4.1902193 .588843-5.067933$

C $5.3123642 .698266-4.816836$

C $7.7666062 .265458-4.724867$

C $9.1402282 .705252-4.929670$

C $9.9667571 .729930-4.434221$

C $9.0705880 .688309-3.884777$

C $8.577034-1.400093-2.573084$

C $9.032640-2.563489-1.807311$

C $7.942486-3.358137-1.585737$

C $\quad 6.797176-2.635084-2.169568$

C $4.345156-2.244255-2.420749$

C $2.967365-2.613281-2.119193$

C $2.200233-1.511950-2.383902$

C $3.089237-0.496372-2.926670$

C $2.6951280 .720713-3.471270$

H $1.6364360 .950327-3.460345$

C $6.6419603 .007831-5.071659$

H $\quad 6.8205403 .966026-5.543899$

C $9.414982-0.431153-3.129392$

H $\quad 10.461502-0.573867-2.919728$

C $5.463676-3.020763-2.119577$

H $5.247601-3.999832-1.705428$

C $1.6579513 .467057-4.451599$

H $\quad 0.9181752 .702919-4.720276$

H $1.5041644 .303759-5.142346$

C $1.4468123 .960368-3.003990$

H $2.3465204 .528166-2.727516$

H $\quad 1.4033463 .112232-2.311726$

C $\quad 0.2593744 .861013-2.681868$

O $-0.0321414 .977618-1.433268$

O $\quad-0.3511275 .519070-3.617003$

N $\quad-3.1758029 .968793-3.036974$

$\mathrm{H} \quad-2.6695489 .106135-2.813121$

C $-4.53444310 .003545-2.813055$

$\mathrm{N} \quad-5.21455611 .163436-2.738881$

H $\quad-6.22658911 .158589-2.868771$

H $\quad-4.80600912 .116011-2.824611$

N $\quad-5.1560758 .855017-2.588954$

H $\quad-4.7729717 .897592-2.762506$

H $\quad-6.1496238 .885960-2.301304$

N $\quad-1.3446135 .228412-6.278026$

H $\quad-1.1258524 .972269-7.232147$

H $\quad-0.6803335 .111285-5.519173$
C $\quad-2.6325455 .559337-5.992974$

N $\quad-3.5206105 .688168-6.965839$

$\mathrm{N} \quad-2.9766535 .773165-4.683352$

$\mathrm{H} \quad-2.2548075 .694043-3.965080$

C $\quad-4.2884726 .060241-4.288949$

O $\quad-4.5209066 .277108-3.027059$

C $\quad-5.2484006 .130187-5.313368$

C $\quad-4.8197875 .936570-6.639037$

N $\quad-6.6150376 .372379-5.030556$

H $\quad-6.8684496 .136657-4.076314$

N $\quad-5.7111345 .999668-7.664111$

H $\quad-5.3812755 .761919-8.592243$

C $\quad-7.5804655 .950937-6.080031$

H $\quad-7.6370244 .854738-6.141909$

C $\quad-7.0765156 .476010-7.442073$

H $\quad-7.7018596 .100202-8.256493$

H $\quad-7.1082247 .572907-7.463879$

C $4.4650203 .917489-0.406762$

H $3.4301343 .785258-0.744404$

H $4.9116542 .917630-0.351853$

N $5.1675244 .700028-1.427128$

H $4.6394405 .396278-1.975231$

C $\quad 6.5234804 .683769-1.612709$

N $7.3447924 .020428-0.828328$

H $7.6932842 .536884-0.100466$

H $7.2513931 .116704-0.606261$

$\mathrm{N} \quad 6.9957525 .426023-2.679736$

H $8.0118235 .463694-2.770124$

H $\quad 6.4455546 .264536-2.948839$

h $4.869471-1.573569-5.997066$

h $4.2941004 .606491-5.444319$

h $\quad 9.3632773 .665373-5.394948$

h $11.0530291 .800936-4.489334$

h $10.052989-2.677290-1.441248$

h $7.829700-4.314950-1.075979$

h $2.668229-3.593855-1.748979$

h $1.136752-1.360686-2.198985$

h $\quad-2.65491510 .642098-3.561673$

h $\quad-8.5735906 .333962-5.845380$

h 4.4559174 .3519180 .592865

\section{****************************************** \\ REACTION 1 SEPTET REACTANT TOTAL QM CHARGE: 0}

***************************************

93

S $\quad 6.153610-1.178801-5.863576$

O $6.0480660 .514963-1.236496$

O $6.8229201 .348605-0.458465$

FE $6.091347-0.034659-3.572567$

N $4.8877811 .558095-4.173605$

$\mathrm{N} \quad 7.7913741 .080490-4.048191$

N $7.269715-1.465355-2.674666$

N $4.385798-0.977529-2.856686$

C $3.5173061 .673271-4.014753$

C $3.0606622 .922276-4.621162$

C $4.1741333 .540215-5.159029$

C $5.3131752 .677306-4.872658$

C $7.7920782 .254129-4.779183$

C $9.1690342 .691483-4.981260$

C $9.9976891 .782356-4.365201$

C $9.1133630 .753444-3.767028$

C $8.630994-1.379947-2.475783$

C $\quad 9.079763-2.558666-1.719388$

C $7.991995-3.366518-1.521649$

C $6.839272-2.651315-2.111402$

C $4.358199-2.265576-2.354337$

C $2.973746-2.634593-2.054876$

C $2.201656-1.533545-2.315463$

C $3.083568-0.505169-2.862928$

C $2.6973330 .725589-3.402039$ 
H $\quad 1.6370730 .951829-3.392369$

C $6.6477972 .954161-5.178831$

H $\quad 6.8211023 .884392-5.710874$

C $9.452014-0.364028-2.985395$

H $10.494601-0.471205-2.733194$

C $\quad 5.492109-3.033317-2.064182$

H $5.278959-4.007983-1.635993$

C $1.6529133 .458819-4.472907$

H $\quad 0.9108782 .674789-4.666207$

H $\quad 1.4723334 .250012-5.209782$

C $1.4851604 .037089-3.050801$

H $2.3515324 .693211-2.881587$

H $\quad 1.5566473 .232643-2.311498$

C $0.2522044 .862443-2.702065$

O $\quad-0.0454294 .929473-1.451007$

O $\quad-0.3802735 .526407-3.619234$

$\mathrm{N} \quad-3.1797769 .970425-3.037802$

H $\quad-2.6725469 .107630-2.816317$

C $-4.53811510 .003428-2.811893$

$\mathrm{N} \quad-5.21833211 .163370-2.733163$

$\mathrm{H} \quad-6.23046911 .158454-2.862357$

H $\quad-4.80978912 .115651-2.821062$

N $\quad-5.1579998 .854369-2.588732$

H $\quad-4.7752537 .896051-2.763290$

H $\quad-6.1519778 .884243-2.301684$

N $\quad-1.3602125 .240311-6.289204$

H $\quad-1.1447284 .976736-7.242154$

H $\quad-0.6966095 .114065-5.531375$

C $\quad-2.6485305 .566270-5.999654$

$\mathrm{N} \quad-3.5392095 .699738-6.969933$

N $\quad-2.9890965 .772602-4.688127$

H $\quad-2.2657185 .683554-3.971703$

C $\quad-4.2980586 .063540-4.289619$

O $\quad-4.5255726 .280441-3.026211$

C $\quad-5.2608876 .137221-5.310568$

C $-4.8374815 .945949-6.638390$

N $\quad-6.6264766 .381495-5.023123$

$\mathrm{H} \quad-6.8754446 .140398-4.068842$

N $\quad-5.7318496 .010597-7.660726$

H $\quad-5.4040295 .771755-8.589211$

C $\quad-7.5932865 .949344-6.067808$

H $\quad-7.6408614 .852547-6.126737$

C $-7.1012476 .474498-7.433912$

H $\quad-7.7274926 .089503-8.243316$

H $\quad-7.1434007 .571067-7.460859$

C $4.6381963 .850915-0.645626$

H $\quad 3.6025583 .743312-0.982029$

H $5.0633712 .846521-0.619795$

N $\quad 5.3376384 .687213-1.631994$

H $4.7907865 .470980-2.032045$

C $6.6126504 .603555-2.045022$

N $7.4587993 .639464-1.655508$

H $7.1670092 .741291-1.203689$

H $8.3753123 .647204-2.081901$

N $7.0585375 .563016-2.887546$

H $\quad 8.0516235 .585544-3.100358$

H $6.4771296 .406246-3.058850$

h $4.862194-1.631188-6.042279$

h $4.2544004 .515991-5.638073$

h $9.4033383 .573601-5.577108$

h $11.0833031 .876124-4.391941$

h $10.101105-2.685647-1.360496$

h $7.879415-4.334155-1.032700$

h $2.674936-3.623718-1.707874$

h $1.137666-1.386645-2.129957$

h $-2.66040410 .645373-3.561891$

h $-8.5872066 .326032-5.826362$

h 4.6527424 .2751130 .358321

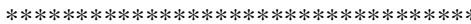

REACTION 1 SEPTET PRODUCT TOTAL QM CHARGE: 0
93

S $\quad 6.131414-1.052679-5.737689$

O $6.1459020 .804569-1.954974$

O 7.7307491 .5580100 .127765

FE $6.0792010 .062497-3.445631$

N $4.8637191 .548200-4.267802$

N $7.8057751 .041840-4.158683$

N $7.250879-1.504617-2.759612$

N $4.352942-0.996269-2.918450$

C $3.4970211 .664424-4.113959$

C $3.0556322 .962751-4.626613$

C $4.1859663 .619582-5.070607$

C $5.3094472 .718523-4.838919$

C $7.8084552 .280791-4.756486$

C $9.1880692 .730666-4.936847$

C $\quad 10.0159901 .749342-4.451282$

C $9.1215330 .684172-3.927495$

C $8.614422-1.446611-2.606297$

C $9.065277-2.603781-1.817419$

C $7.970392-3.386842-1.569555$

C $6.816945-2.668107-2.155809$

C $4.321089-2.265714-2.387770$

C $2.937161-2.630889-2.083516$

C $2.164732-1.541165-2.387071$

C $3.052472-0.528977-2.954301$

C $2.6798780 .698742-3.520732$

$\mathrm{H} \quad 1.6192110 .927257-3.515012$

C $6.6596153 .015581-5.077683$

$\mathrm{H} \quad 6.8381353 .985480-5.528071$

C $9.442480-0.459471-3.172741$

H $\quad 10.489399-0.602171-2.956229$

C $5.463930-3.023265-2.078514$

H $\quad 5.249264-3.987613-1.626318$

C $1.6497733 .497593-4.469118$

H $\quad 0.9063282 .737890-4.740223$

H $\quad 1.4981854 .340302-5.152968$

C $1.4462353 .979470-3.016533$

H $\quad 2.3423824 .556360-2.746940$

H $\quad 1.4206923 .126781-2.329147$

C $\quad 0.2528604 .866077-2.679049$

O $\quad-0.0359514 .965281-1.428691$

O $\quad-0.3632465 .531847-3.605718$

$\mathrm{N} \quad-3.1782459 .971069-3.037334$

$\mathrm{H} \quad-2.6701519 .110120-2.810467$

C $\quad-4.53637110 .005091-2.811923$

$\mathrm{N} \quad-5.21688111 .164705-2.736365$

H $\quad-6.22902611 .159658-2.865457$

H $\quad-4.80853612 .117382-2.822932$

N $\quad-5.1569798 .856490-2.586792$

H $\quad-4.7745307 .898879-2.759822$

H $\quad-6.1508088 .886793-2.299567$

$\mathrm{N} \quad-1.3519545 .239095-6.274234$

H $-1.1302984 .983712-7.227670$

H $\quad-0.6886765 .123013-5.514521$

C $-2.6412995 .566157-5.990686$

$\mathrm{N} \quad-3.5284705 .695787-6.964423$

N $-2.9868915 .775504-4.680932$

H $\quad-2.2659755 .695041-3.961723$

C $-4.2985456 .063418-4.287286$

O $\quad-4.5313876 .277743-3.025097$

C $\quad-5.2572666 .135880-5.312755$

C $-4.8280825 .943947-6.638365$

N $\quad-6.6239286 .380581-5.031079$

H $\quad-6.8780036 .139770-4.078177$

N $\quad-5.7181456 .009665-7.664509$

H $\quad-5.3879415 .769560-8.591833$

C $\quad-7.5876755 .955080-6.081057$

H $\quad-7.6403814 .858665-6.142681$

C $\quad-7.0859726 .480674-7.443424$

H $\quad-7.7099566 .101721-8.257324$

H $\quad-7.121722$ 7.577507 -7.467357
C $4.4646403 .910412-0.408198$

H $3.4294283 .780404-0.745994$

$\mathrm{H} \quad 4.9092302 .909505-0.354987$

N $5.1672074 .693858-1.427913$

H $4.6395535 .394353-1.971123$

C $6.5232224 .676891-1.613917$

N $7.3436654 .014139-0.828757$

H $7.6893252 .529328-0.101769$

H $7.2677661 .105073-0.614325$

N $6.9969375 .418109-2.681150$

$\mathrm{H} \quad 8.0134005 .454250-2.770044$

H $\quad 6.4484846 .257491-2.950703$

h $4.881743-1.576850-5.998294$

h $4.2963364 .643033-5.428984$

h $9.4110293 .697185-5.388728$

h $11.1026791 .823140-4.492841$

h $10.085381-2.711379-1.448805$

h $7.854835-4.335931-1.046159$

h $2.642063-3.606914-1.698342$

h $1.101954-1.386528-2.200912$

h $\quad-2.65773810 .645668-3.560747$

h $\quad-8.5813146 .335694-5.844663$

h 4.4570554 .3438280 .591882

\section{*********************************** \\ REACTION 2 SINGLET \\ REACTANT \\ TOTAL QM CHARGE: 1 \\ ***************************************}

94

S $\quad 6.210358-1.046764-5.460987$

O $6.0857420 .850011-1.511692$

O $7.3530300 .817061-0.655171$

H $7.710111-0.085579-0.820680$

FE $6.151797-0.019542-3.225345$

N $4.9609521 .440087-3.973108$

$\mathrm{N} \quad 7.8205430 .973707-3.832521$

N $7.319306-1.488661-2.483516$

$\mathrm{N} \quad 4.493738-1.033276-2.688461$

C $3.5918371 .558850-3.856049$

C $3.1170142 .767968-4.519580$

C $4.2299163 .382501-5.054271$

C $5.3700742 .543718-4.710371$

C $7.8345642 .128832-4.589782$

C $9.2045112 .573952-4.805734$

C $10.0338911 .694044-4.155599$

C $9.1498390 .687345-3.538969$

C $8.686370-1.431424-2.266100$

C $9.144168-2.634281-1.570861$

C $8.063995-3.466350-1.446768$

C $6.916127-2.724439-2.001543$

C $4.451520-2.331256-2.223291$

C $3.073780-2.720027-1.919134$

C $2.296555-1.619644-2.150243$

C $3.177090-0.585604-2.680373$

C $2.7723030 .624973-3.228051$

H $1.7107280 .841626-3.230382$

C $6.6977802 .825514-5.005461$

H $\quad 6.8723233 .739770-5.562701$

C $9.503448-0.392660-2.724486$

H $10.530067-0.447599-2.403665$

C $5.578289-3.116380-1.966389$

H $5.365235-4.114796-1.600518$

C $1.6877853 .263185-4.445043$

$\mathrm{H} \quad 0.9810222 .455595-4.668000$

H $1.5261954 .034770-5.204611$

C $1.4243853 .858986-3.045115$

H $2.2830524 .508268-2.825291$

H $1.4258093 .068215-2.288344$

C $0.1830204 .712545-2.800312$

O $\quad-0.2348084 .764896-1.586119$ 
O $\quad-0.3117495 .426100-3.763172$

N $\quad-3.09517710 .073305-3.206128$

H $\quad-2.4808329 .267880-3.003336$

C $-4.4394179 .970364-2.937231$

N $\quad-5.28871211 .005020-3.027618$

$\mathrm{H} \quad-6.278583 \quad 10.783544-2.971908$

H $\quad-5.03602812 .018137-3.036479$

N $\quad-4.9117498 .787149-2.548215$

H $\quad-4.4590307 .859840-2.670565$

H $\quad-5.9112168 .749100-2.328968$

$\mathrm{N} \quad-1.2864675 .360191-6.533901$

H $\quad-1.1310035 .111481-7.504893$

H $\quad-0.5930455 .177060-5.820110$

C $-2.5493405 .707404-6.174934$

$\mathrm{N} \quad-3.4751315 .928804-7.097500$

N $\quad-2.8316425 .840208-4.841390$

H $\quad-2.0757425 .690009-4.162133$

C $-4.1096756 .118352-4.361806$

O $\quad-4.2755946 .239408-3.087166$

C $\quad-5.1254896 .274800-5.343288$

C $-4.7521336 .177473-6.692338$

N $\quad-6.4660616 .495755-4.979790$

H $\quad-6.6855976 .206902-4.032064$

N $\quad-5.6734436 .389235-7.696944$

H $\quad-5.5144425 .901379-8.576621$

C $\quad-7.4844366 .192810-6.019609$

H $\quad-7.5423525 .106043-6.176622$

C $\quad-7.0344706 .836229-7.350488$

H $\quad-7.6972946 .540707-8.167234$

H $\quad-7.0530497 .929523-7.270817$

C $4.2822553 .860895-0.759347$

H $3.2398093 .819087-1.075527$

H $4.6466472 .830853-0.762882$

N $5.0137554 .680687-1.745937$

H $\quad 4.5077045 .475993-2.168938$

C $6.3300984 .596511-2.010572$

N $7.0652943 .561197-1.570020$

H $6.6431092 .651803-1.371289$

H $8.0553033 .553254-1.757016$

N $6.9085405 .578243-2.724108$

H $7.9160915 .570572-2.874738$

H $6.3576636 .432635-2.948419$

h $4.988108-1.597037-5.789090$

h $4.3006134 .348401-5.554376$

h $9.4433263 .438958-5.424441$

h $\quad 11.1197541 .785987-4.178296$

h $10.158179-2.754880-1.189673$

h $7.961733-4.464049-1.019912$

h $2.787613-3.714608-1.577106$

h $1.229286-1.477548-1.980501$

h $-2.64709910 .778037-3.756174$

h $-8.4700786 .543065-5.713182$

h 4.3318864 .2693750 .249983

\section{$* * * * * * * * * * * * * * * * * * * * * * * * * * * * * * * * * *$ \\ REACTION 2 SINGLET PRODUCT TOTAL QM CHARGE: 1}

$* * * * * * * * * * * * * * * * * * * * * * * * * * * * * * * * * * *$

94

S $\quad 6.143273-1.111523-5.704278$

O $5.8877340 .706986-1.886288$

O $7.4619301 .878000-0.055510$

H $\quad 6.9872721 .311543-0.702884$

FE $5.997913-0.043897-3.356916$

$\mathrm{N} \quad 4.8186311 .356677-4.219520$

N $7.6519450 .960048-3.965664$

N $7.168532-1.512841-2.634558$

N $\quad 4.361285-1.125073-2.921808$

C $3.4519161 .478667-4.067337$

C $\quad 2.9764302 .717888-4.673650$

C $4.0824463 .347962-5.199872$
C $\quad 5.2243872 .492158-4.901792$

C $7.6775742 .116991-4.722028$

C $\quad 9.0512412 .583138-4.888587$

C $9.8639801 .730784-4.189203$

C $8.9667280 .718527-3.591079$

C $8.506687-1.397683-2.335210$

C $8.987071-2.599061-1.638254$

C $7.933195-3.464401-1.551607$

C $6.782311-2.757258-2.157176$

C $4.327066-2.402552-2.413262$

C $2.957774-2.770579-2.049653$

C $2.181939-1.671901-2.289886$

C $3.049423-0.662176-2.886524$

C $2.6410020 .546951-3.429066$

H $\quad 1.5821740 .774032-3.397336$

C $6.5514022 .801561-5.169450$

H $\quad 6.7283573 .738172-5.685757$

C $9.304645-0.319140-2.721681$

H $\quad 10.293601-0.296688-2.295075$

C $5.458175-3.176746-2.142021$

H $5.252630-4.167922-1.753927$

C $\quad 1.5590233 .226272-4.535727$

H $\quad 0.8340642 .425953-4.725002$

H $1.3720844 .000306-5.287356$

C $1.3639043 .823149-3.123246$

H $2.2331584 .471327-2.941328$

H $1.4011683 .033936-2.365753$

C $\quad 0.1275034 .669275-2.834935$

O $\quad-0.2613164 .724515-1.615047$

O $\quad-0.4080015 .379138-3.788203$

$\mathrm{N} \quad-3.09577410 .086050-3.224011$

H $\quad-2.4749309 .282428-3.024850$

C $-4.4274699 .986280-2.913896$

$\mathrm{N} \quad-5.28880611 .008377-2.990954$

H $\quad-6.27583010 .780429-2.905825$

$\mathrm{H} \quad-5.04441412 .025208-3.019137$

N $\quad-4.8814778 .799331-2.499710$

$\mathrm{H} \quad-4.4024887 .896492-2.593939$

H $\quad-5.8686788 .744873-2.244083$

$\mathrm{N} \quad-1.3538015 .344748-6.502327$

H $\quad-1.1791925 .119863-7.477239$

H $\quad-0.6591675 .178744-5.779166$

C $-2.6093965 .682993-6.151631$

$\mathrm{N} \quad-3.5320225 .911036-7.088621$

$\mathrm{N} \quad-2.8996745 .801886-4.815464$

H $\quad-2.1296355 .654578-4.140309$

C $-4.1806386 .052914-4.341919$

O $\quad-4.3883256 .162080-3.084736$

C $\quad-5.1981956 .192600-5.344027$

C $-4.8113746 .127053-6.704209$

N $\quad-6.5233276 .340684-4.991841$

$\mathrm{H} \quad-6.7463216 .143904-4.021657$

N $\quad-5.7378236 .325800-7.692429$

H $\quad-5.5547825 .906667-8.603972$

C $-7.5611196 .122561-6.031143$

H $\quad-7.6511515 .041474-6.200931$

C $\quad-7.0975046 .782094-7.347722$

H $\quad-7.7606976 .499006-8.167546$

H $\quad-7.1061237 .875108-7.258824$

C $4.6738344 .015678-1.035321$

H $3.6432583 .839930-1.358013$

H $5.1873573 .051504-1.018471$

$\mathrm{N} \quad 5.3082584 .889353-2.026421$

H $4.7193565 .591858-2.490988$

C $6.6442584 .900284-2.309426$

N $7.4888174 .124563-1.645936$

H $7.4787582 .788818-0.450194$

H $\quad 8.4526224 .269993-1.968160$

N $7.0292785 .752860-3.318419$

H $\quad 8.0295375 .893417-3.421402$

H $6.3877196 .536602-3.540878$

h $4.915782-1.652838-6.027681$

h $4.1634704 .328609-5.668736$ h $\quad 9.2959913 .444985-5.509380$

h $10.9488081 .833697-4.164216$

h $10.009827-2.723717-1.282590$

h $7.837587-4.460149-1.118697$

h $2.685862-3.741059-1.634563$

h $1.125437-1.512178-2.074578$

h $-2.65169610 .801609-3.763215$

h $\quad-8.5329836 .495359-5.707773$

h $4.6731614 .419272-0.022810$

\section{REACTION 2 TRIPLET \\ REACTANT \\ TOTAL QM CHARGE: 1 \\ $* * * * * * * * * * * * * * * * * * * * * * * * * * * * * * * * * *$}

94

S $\quad 6.211536-1.043631-5.456529$

O $6.0837720 .847994-1.518741$

O $7.3474770 .816165-0.656679$

H $7.708346-0.084369-0.824970$

FE $6.151634-0.017522-3.228332$

N $4.9615781 .441696-3.970930$

N $7.8207990 .975189-3.831706$

N $7.320857-1.487581-2.482546$

N $\quad 4.493545-1.032440-2.687967$

C $\quad 3.5927151 .559397-3.855567$

C $3.1178902 .768285-4.519830$

C $\quad 4.2306753 .383440-5.053404$

C $5.3711552 .545712-4.708109$

C $7.8355502 .129485-4.588263$

C $9.2051072 .574322-4.805441$

C $\quad 10.0347441 .694414-4.155734$

C $9.1509310 .687193-3.538165$

C $8.686621-1.431453-2.265637$

C $9.145492-2.634041-1.570966$

C $8.065139-3.466068-1.446756$

C $6.917224-2.723773-2.000918$

C $4.452079-2.329620-2.222475$

C $3.074223-2.718984-1.918660$

C $2.296638-1.618917-2.149704$

C $3.177619-0.585041-2.679867$

C $2.7726910 .625473-3.228065$

H $\quad 1.7110670 .841936-3.230649$

C $\quad 6.6983342 .827122-5.003499$

H $\quad 6.8729033 .740625-5.561841$

C $9.504522-0.392050-2.724592$

H $\quad 10.531381-0.448123-2.404586$

C $5.579350-3.115001-1.965631$

H $5.366182-4.113749-1.600696$

C $1.6887553 .262752-4.446350$

H $\quad 0.9813522 .455467-4.668575$

H $\quad 1.5261054 .034612-5.205242$

C $1.4266723 .857615-3.046252$

H $\quad 2.2844154 .507687-2.825116$

H $1.4275943 .068044-2.288262$

C $\quad 0.1853374 .711068-2.800525$

O $\quad-0.2327914 .762248-1.586541$

O $\quad-0.3101815 .425305-3.762602$

N $\quad-3.09554610 .072952-3.206524$

H $\quad-2.4807369 .267837-3.003902$

C $-4.4398039 .970259-2.937670$

N $\quad-5.28915111 .004882-3.028305$

H $\quad-6.27914510 .784083-2.971920$

$\mathrm{H} \quad-5.03664412 .018079-3.036820$

$\mathrm{N} \quad-4.9122718 .787132-2.548267$

H $\quad-4.4593207 .860345-2.670280$

$\mathrm{H} \quad-5.9117008 .748595-2.328903$

N $\quad-1.2860565 .360645-6.533629$

H $\quad-1.1304155 .111061-7.504344$

H $\quad-0.5927315 .177878-5.819570$

C $\quad-2.5488875 .707163-6.174783$ 
N $\quad-3.4749415 .928720-7.097394$

$\mathrm{N} \quad-2.8311565 .839709-4.841251$

H $\quad-2.0754945 .689675-4.161753$

C $-4.1091266 .118194-4.361765$

O $\quad-4.2749256 .238535-3.087194$

C $\quad-5.1250916 .274840-5.343408$

C $-4.7517816 .177047-6.692506$

N $\quad-6.4653596 .495930-4.980123$

H $\quad-6.6845186 .207307-4.032085$

N $\quad-5.6730926 .389342-7.696659$

H $\quad-5.5141645 .901485-8.576346$

C $\quad-7.4841246 .193261-6.020127$

H $\quad-7.5420985 .106534-6.176764$

C $\quad-7.0339576 .836385-7.350701$

H $\quad-7.6967446 .540669-8.167417$

H $\quad-7.0523797 .929636-7.271282$

C $4.2820063 .860756-0.759146$

H $3.2396243 .819208-1.075523$

H $4.6462312 .830614-0.763237$

N $5.0136224 .680457-1.745626$

H $4.5075865 .475733-2.168583$

C $6.3299264 .595937-2.010150$

N $7.0647593 .560263-1.569909$

H $6.6422102 .650937-1.371621$

H $8.0547393 .551471-1.757107$

N $6.9085435 .577417-2.723891$

H $7.9160685 .569791-2.874294$

H $6.3575446 .431698-2.948395$

h $4.990005-1.594724-5.785928$

h $4.3010244 .349022-5.554173$

h $9.4437763 .439222-5.424352$

h $\quad 11.1206191 .786043-4.179100$

h $10.159485-2.754830-1.189792$

h $7.962688-4.463695-1.019777$

h $2.788151-3.713791-1.577209$

h $1.229345-1.476835-1.980100$

h $\quad-2.64760510 .777914-3.756388$

h $\quad-8.4697326 .543392-5.713449$

h 4.3317604 .2689560 .250291

$* * * * * * * * * * * * * * * * * * * * * * * * * * * * * * * * * * * * *$

REACTION 2 TRIPLET PRODUCT TOTAL QM CHARGE: 1

$* * * * * * * * * * * * * * * * * * * * * * * * * * * * * * * * * * * *$

94

S $\quad 6.142693-1.107377-5.700712$

O $5.8890910 .700826-1.886161$

O $7.4640661 .881146-0.058255$

H $6.9878971 .314875-0.703953$

FE $5.999248-0.045094-3.359364$

$\mathrm{N} \quad 4.8203661 .359335-4.216427$

N $7.6533890 .960489-3.965082$

N $7.170509-1.514998-2.638230$

$\mathrm{N} \quad 4.362555-1.123670-2.921504$

C $3.4529041 .480457-4.065895$

C $2.9767162 .721847-4.672618$

C $4.0819903 .351430-5.197380$

C $5.2252722 .495815-4.895438$

C $7.6786412 .119171-4.719458$

C $9.0538982 .583580-4.889342$

C $9.8663041 .730697-4.191757$

C $8.9673920 .718516-3.592429$

C $8.509826-1.398547-2.336736$

C $8.989586-2.600728-1.638779$

C $7.935518-3.464792-1.551818$

C $6.784944-2.756433-2.159417$

C $4.328860-2.402411-2.411981$

C $2.957514-2.770640-2.048042$

C $2.183084-1.672166-2.289336$

C $3.052881-0.661600-2.884997$

C $2.6425780 .548580-3.429419$
H $\quad 1.5836640 .775016-3.398089$

C $6.5529972 .806490-5.162991$

H $\quad 6.7298933 .745010-5.675358$

C $9.306563-0.319155-2.721118$

H $10.295369-0.294006-2.294083$

C $5.458402-3.176500-2.141998$

H $5.253824-4.167744-1.753988$

C $1.5590523 .228830-4.534345$

H $0.8348322 .428687-4.727001$

H $\quad 1.3726194 .004753-5.283802$

C $1.3653393 .823494-3.120844$

H $\quad 2.2348054 .471010-2.938574$

H $1.4029523 .035102-2.362515$

C $0.1290524 .670368-2.832208$

O $\quad-0.2591274 .723433-1.611990$

O $\quad-0.4036625 .382049-3.784472$

$\mathrm{N} \quad-3.09605010 .084625-3.223783$

H $\quad-2.4750999 .281111-3.024710$

C $\quad-4.4281069 .983762-2.914495$

$\mathrm{N} \quad-5.28929411 .006454-2.990767$

$\mathrm{H} \quad-6.27630010 .778010-2.906554$

H $\quad-5.04480812 .022913-3.018428$

$\mathrm{N} \quad-4.8820478 .796335-2.502889$

H $\quad-4.4060237 .891272-2.602355$

H $\quad-5.8696628 .741724-2.248916$

N $\quad-1.3531175 .346379-6.505461$

H $\quad-1.1806025 .120490-7.480293$

$\mathrm{H} \quad-0.6582215 .180320-5.783556$

C $\quad-2.6097275 .685041-6.153447$

N $\quad-3.5323965 .912969-7.088689$

$\mathrm{N} \quad-2.8990425 .803926-4.817550$

H $\quad-2.1315265 .655307-4.141364$

C $\quad-4.1799306 .055825-4.342662$

O $\quad-4.3822746 .166715-3.083186$

C $\quad-5.1977796 .195456-5.342706$

C $\quad-4.8119726 .129933-6.701557$

N $\quad-6.5246076 .348479-4.989937$

H $\quad-6.7480486 .147223-4.021029$

N $\quad-5.7382546 .329030-7.691215$

H $\quad-5.5552425 .906553-8.600803$

C $\quad-7.5607986 .124804-6.029316$

H $\quad-7.6497275 .043637-6.201021$

C $\quad-7.0978736 .784064-7.346820$

$\mathrm{H} \quad-7.7607066 .500025-8.166786$

H $\quad-7.1081447 .877054-7.258058$

C $4.6703724 .015543-1.035458$

H $3.6379273 .841363-1.354368$

H $5.1808583 .049632-1.017197$

N $5.3063574 .881837-2.030864$

H $4.7217765 .586090-2.495819$

C $6.6450644 .890775-2.309852$

N $7.4895554 .119479-1.647051$

H $7.4792722 .791983-0.458947$

H $8.4532064 .268059-1.966895$

N $7.0257415 .744410-3.323620$

H $8.0251575 .894147-3.419960$

H $6.3833216 .530158-3.536640$

h $4.916371-1.650622-6.025316$

h $4.1638624 .332061-5.666129$

h $9.2982813 .446509-5.508774$

h $\quad 10.9511871 .832973-4.166581$

h $10.012383-2.725237-1.283179$

h $7.838730-4.459835-1.117549$

h $2.685287-3.741525-1.634107$

h $1.126632-1.511284-2.074644$

h $\quad-2.65248810 .800423-3.763096$

h $\quad-8.5334396 .496508-5.707022$

h $4.6714614 .418957-0.022876$

$* * * * * * * * * * * * * * * * * * * * * * * * * * * * * * * * * * *$

REACTION 2 QUINTET

REACTANT

TOTAL QM CHARGE: 1
*** $* * * * * * * * * * * * * * * * * * * * * * * * * * * * * * * *$

94

S $\quad 6.193995-1.186037-5.701673$

O $6.2166560 .995558-1.267828$

O $7.4861960 .862763-0.440497$

H $7.818871-0.034553-0.671981$

FE $6.1966640 .043034-3.121536$

N $5.0155441 .444868-3.965884$

$\mathrm{N} \quad 7.8481880 .993484-3.816032$

$\mathrm{N} \quad 7.345661-1.451907-2.435285$

N 4.542204-1.017691-2.672019

C $3.6358661 .557348-3.854959$

C $3.1632962 .760961-4.521118$

C $4.2767293 .380412-5.054311$

C $5.4193172 .555386-4.706303$

C $7.8695712 .144985-4.589067$

C $9.2401742 .572155-4.813121$

C $10.0649221 .695106-4.152899$

C $9.1812100 .703743-3.516992$

C $8.716816-1.390804-2.222275$

C $9.175701-2.596931-1.539684$

C $8.099100-3.436852-1.423622$

C $6.947566-2.699998-1.965051$

C $4.496217-2.322384-2.205403$

C $3.117822-2.707661-1.917507$

C $2.341870-1.604831-2.148971$

C $3.222372-0.570678-2.672029$

C $2.8145720 .636092-3.223146$

H $\quad 1.7535540 .851928-3.229216$

C $6.7416652 .844224-5.008133$

H $\quad 6.9146173 .757020-5.566985$

C $9.535779-0.358838-2.685622$

H $10.563310-0.412826-2.368082$

C $\quad 5.615769-3.105337-1.939395$

H $5.404741-4.104231-1.575400$

C $1.7314663 .252026-4.452113$

H $\quad 1.0281202 .441659-4.675168$

H $1.5684824 .021949-5.213081$

C $1.4622233 .849933-3.053165$

$\mathrm{H} \quad 2.3142834 .507040-2.831793$

H $\quad 1.4672063 .061770-2.293994$

C $0.2119524 .694158-2.811152$

O $-0.2088344 .743453-1.597663$

O $\quad-0.2874645 .403834-3.774299$

N $\quad-3.09718210 .071311-3.214060$

H $\quad-2.4812579 .267035-3.011909$

C $\quad-4.4409089 .968349-2.942122$

N $\quad-5.28983111 .003682-3.029027$

H $\quad-6.27974710 .782819-2.971002$

H $\quad-5.03697212 .016678-3.037169$

N $\quad-4.9127908 .784681-2.553989$

H $\quad-4.4587867 .857638-2.676030$

H $\quad-5.9121668 .746195-2.334016$

N $\quad-1.2744915 .356802-6.537389$

H $\quad-1.1216365 .105872-7.508120$

H $\quad-0.5813945 .169769-5.824044$

C $\quad-2.5369955 .704003-6.176294$

$\mathrm{N} \quad-3.4639515 .926956-7.097042$

$\mathrm{N} \quad-2.8172225 .836140-4.842304$

H $\quad-2.0618045 .681054-4.163968$

C $\quad-4.0938546 .119180-4.361076$

O $\quad-4.2562446 .240541-3.085514$

C $\quad-5.1108026 .278958-5.340782$

C $\quad-4.7399606 .178057-6.689812$

$\mathrm{N} \quad-6.4513916 .504879-4.975367$

H $\quad-6.6692306 .212792-4.028019$

N $\quad-5.6619196 .390932-7.694203$

H $\quad-5.5050275 .899067-8.571951$

C $\quad-7.4705046 .195955-6.013627$

H $\quad-7.5270555 .108448-6.166601$

C $\quad-7.0234076 .835890-7.347067$ 


\begin{abstract}
H $\quad-7.6867856 .535898-8.161828$
H $\quad-7.0445307 .929368-7.270996$

C $4.2957203 .873883-0.727871$

H $3.2549463 .813948-1.048006$

H $\quad 4.6766432 .849630-0.712998$

N $5.0203984 .690660-1.722089$

H $\quad 4.5071745 .474100-2.157424$

C $6.3409684 .623750-1.975967$

N $7.0899823 .611595-1.509995$

H $\quad 6.6803362 .687946-1.299127$

H $8.0831073 .628954-1.681081$

N $6.9052275 .600461-2.709312$

H $7.9114505 .595445-2.866167$

H $6.3472296 .446187-2.946983$

h $\quad 4.926445-1.678485-5.936533$

h $4.3430344 .345169-5.557217$

h $9.4845033 .426821-5.443926$

h $\quad 11.1512261 .779738-4.182007$

h $10.191244-2.718766-1.162994$

h $8.004198-4.440607-1.009454$

h $2.826941-3.703450-1.583052$

h $1.273171-1.465149-1.986368$

h $-2.65066810 .779040-3.761523$

h $\quad-8.4566316 .544524-5.706839$

h $\quad 4.3344254 .2927000 .277694$
\end{abstract}

$* * * * * * * * * * * * * * * * * * * * * * * * * * * * * * * * * * *$
REACTION 2 QUINTET
PRODUCT
TOTAL QM CHARGE: 1
$* * * * * * * * * * * * * * * * * * * * * * * * * * * * * * * * * *$

94

S $\quad 6.144708-1.100809-5.695323$

O $5.9038930 .700260-1.889086$

O $7.4644151 .884690-0.056431$

H $\quad 6.9953651 .312985-0.702888$

FE $5.993518-0.046683-3.364094$

$\mathrm{N} \quad 4.8184201 .358426-4.217083$

$\mathrm{N} \quad 7.6531690 .961825-3.966550$

N 7.168423-1.514256-2.635489

N $4.362075-1.122155-2.920402$

C $3.4521601 .481008-4.065954$

C $2.9774422 .720315-4.672262$

C $4.0836293 .350147-5.198194$

C $5.2255132 .494890-4.899146$

C $7.6791512 .118790-4.721412$

C $9.0531912 .583867-4.889171$

C $9.8654601 .730669-4.190039$

C $8.9669920 .718996-3.592219$

C $8.507928-1.397859-2.336277$

C $8.988510-2.599081-1.639225$

C $7.934521-3.463726-1.551208$

C $6.783537-2.756831-2.157865$

C $4.328151-2.401601-2.412843$

C $2.958933-2.768925-2.049132$

C $2.182883-1.670757-2.290115$

C $3.050066-0.659867-2.884738$

C $2.6410460 .548509-3.428601$

H $\quad 1.5820010 .774757-3.397900$

C $6.5523022 .804903-5.166265$

H $\quad 6.7293063 .743778-5.678271$

C $9.305197-0.319842-2.723309$

H $\quad 10.294908-0.297827-2.298373$

C $5.458412-3.175928-2.142415$

H $5.252487-4.167112-1.754504$

C $1.5597583 .228219-4.535160$

H $\quad 0.8350542 .428150-4.726303$

H $\quad 1.3736924 .003090-5.286126$

C $1.3629393 .824300-3.122703$

H $2.2315764 .473250-2.940713$

H $1.4012653 .036210-2.364079$
C $\quad 0.1260994 .669831-2.834039$

O $\quad-0.2631334 .723933-1.614343$

O $\quad-0.4089315 .380821-3.786759$

$\mathrm{N} \quad-3.09651410 .085668-3.223855$

H $\quad-2.4757459 .282280-3.024063$

C $-4.4283319 .986122-2.913945$

$\mathrm{N} \quad-5.28953611 .008346-2.991053$

$\mathrm{H} \quad-6.27658110 .780301-2.906016$

$\mathrm{H} \quad-5.04518312 .025079-3.019060$

N $\quad-4.8825778 .798905-2.500898$

H $\quad-4.4037697 .896192-2.596521$

H $\quad-5.8703128 .744562-2.246519$

$\mathrm{N} \quad-1.3533745 .346075-6.504185$

$\mathrm{H} \quad-1.1800215 .120156-7.479167$

H $\quad-0.6582005 .179943-5.781523$

C $\quad-2.6088525 .683959-6.152769$

N $\quad-3.5320435 .912111-7.089151$

N $\quad-2.8984435 .802954-4.816449$

H $\quad-2.1282145 .653821-4.141687$

C $-4.1791616 .053133-4.342232$

O $\quad-4.3860846 .162808-3.084883$

C $\quad-5.1974886 .193005-5.343925$

C $-4.8110746 .128986-6.704160$

N $\quad-6.5223116 .344769-4.990924$

H $\quad-6.7449936 .145119-4.021207$

N $\quad-5.7377496 .327913-7.692222$

H $\quad-5.5556375 .907185-8.603161$

C $\quad-7.5603716 .124177-6.029954$

H $\quad-7.6485775 .042775-6.199121$

C $-7.0974296 .783583-7.346756$

H $\quad-7.7609756 .500515-8.166366$

H $\quad-7.1066137 .876589-7.257616$

C $4.6709364 .014001-1.034968$

H $3.6395253 .839183-1.355912$

H $5.1819153 .048372-1.016216$

$\mathrm{N} \quad 5.3053464 .884193-2.028949$

H $\quad 4.7170205 .587312-2.493077$

C $6.6417424 .896277-2.311028$

N $7.4871454 .123328-1.646069$

H $7.4782492 .794223-0.455694$

H $8.4507964 .268850-1.968223$

$\mathrm{N} 7.0257145 .748213-3.321584$

H $\quad 8.0257035 .893673-3.420763$

H $\quad 6.3836326 .532267-3.541643$

h $4.919601-1.645809-6.021566$

h $4.1645714 .331252-5.666113$

h $9.2982653 .446620-5.508576$

h $10.9503651 .832759-4.165001$

h $10.011525-2.724299-1.284501$

h $7.838232-4.458816-1.116937$

h $2.686999-3.739800-1.634982$

h $1.126321-1.510671-2.075364$

h $-2.65224010 .801070-3.763107$

h $\quad-8.5326206 .496333-5.707003$

h $4.6716964 .418911-0.022983$

*****************************************

REACTION 2 SEPTET REACTANT TOTAL QM CHARGE: 1

***************************************

94

S $\quad 6.197765-1.112847-5.639405$

O $6.2543100 .974297-1.295387$

O $7.5115270 .835366-0.459896$

H $7.864793-0.050854-0.708897$

FE $6.2039240 .045277-3.110611$

$\mathrm{N} \quad 4.9848811 .480857-3.987933$

N $7.9042981 .008108-3.845282$

N $7.382816-1.506630-2.432720$

N $4.495399-1.052384-2.668394$

C $3.6074721 .566411-3.896286$
C $3.1429602 .784446-4.554080$

C $4.2651433 .419089-5.058175$

C $5.4116192 .595517-4.701544$

C $7.9039372 .174900-4.586481$

C $\quad 9.2795152 .600738-4.814514$

C $\quad 10.1094241 .701032-4.188621$

C $9.2288160 .689684-3.565742$

C $8.754200-1.444732-2.255301$

C $9.207631-2.645155-1.549337$

C $8.121491-3.468566-1.393947$

C $6.963657-2.729241-1.930834$

C $4.469813-2.341779-2.170760$

C $3.084570-2.728284-1.892383$

C $2.302506-1.639182-2.170545$

C $3.182370-0.605447-2.709176$

C $2.7938320 .609412-3.283235$

H $\quad 1.7297620 .814677-3.308973$

C $6.7527462 .875276-4.977030$

H $\quad 6.9266343 .798484-5.520968$

C $9.566349-0.408215-2.754250$

H $10.601064-0.487902-2.463834$

C $5.612311-3.105051-1.885603$

H $\quad 5.400849-4.092489-1.490072$

C $\quad 1.7128503 .278570-4.483632$

$\mathrm{H} \quad 1.0067302 .474049-4.718442$

H $\quad 1.5530864 .063007-5.230230$

C $1.4493473 .852025-3.072774$

H $2.3071374 .498179-2.840674$

H $\quad 1.4504743 .050134-2.327946$

C $\quad 0.2077704 .703270-2.814358$

O $\quad-0.2066444 .743725-1.598687$

O $\quad-0.2901735 .426394-3.768081$

N $\quad-3.09775810 .072578-3.211522$

H $\quad-2.4826259 .268152-3.008606$

C $\quad-4.4424459 .968891-2.942979$

N $\quad-5.29128411 .004358-3.031884$

H $\quad-6.28130010 .783519-2.976375$

H $\quad-5.03835912 .017312-3.039271$

$\mathrm{N} \quad-4.9146778 .785508-2.555653$

$\mathrm{H} \quad-4.4617217 .856904-2.679771$

H $\quad-5.9150128 .747678-2.339016$

$\mathrm{N}-1.2718935 .365280-6.540381$

H $\quad-1.1196865 .110858-7.510463$

H $\quad-0.5777495 .182087-5.827220$

C $\quad-2.5353865 .710692-6.178875$

N $\quad-3.4634785 .930044-7.098443$

$\mathrm{N} \quad-2.8147015 .844206-4.844928$

H $\quad-2.0587565 .693885-4.166240$

C $-4.0910426 .127076-4.362845$

O $\quad-4.2499786 .249289-3.086143$

C $\quad-5.1087136 .285936-5.340455$

C $-4.7392876 .182676-6.689160$

$\mathrm{N} \quad-6.4502066 .516215-4.973824$

H $\quad-6.6668446 .217912-4.027966$

N $\quad-5.6617496 .394562-7.693809$

H $\quad-5.5062535 .899066-8.569639$

C $-7.4681316 .200222-6.011393$

H $\quad-7.5211485 .112472-6.163783$

C $\quad-7.0229506 .839211-7.346153$

H $\quad-7.6868486 .538509-8.160425$

H $\quad-7.0442837 .932659-7.270638$

C $4.2919903 .876797-0.733104$

H $3.2528573 .821508-1.058999$

H $4.6700752 .851493-0.720485$

N $5.0229714 .694572-1.721929$

H $\quad 4.5085615 .475042-2.162060$

C $6.3444864 .633086-1.967854$

N $7.0995813 .629041-1.490232$

H $\quad 6.6970812 .709635-1.269031$

H $8.0923313 .649586-1.663423$

N $6.9081045 .605297-2.706145$

H $7.9152265 .602456-2.859627$

H $6.3482976 .448420-2.950169$ 
h $\quad 4.949343-1.636990-5.905981$

h $4.3354774 .386490-5.555423$

h $9.5187933 .467189-5.431016$

h $11.1956211 .785477-4.221953$

h $10.222442-2.757283-1.167692$

h $8.023221-4.465158-0.963582$

h $2.797385-3.720345-1.543949$

h $1.235445-1.492968-2.003005$

h $\quad-2.65103310 .779084-3.760391$

h $\quad-8.4550576 .547220-5.705392$

h 4.3274914 .2925130 .273865

REACTION 2 SEPTET PRODUCT TOTAL QM CHARGE: 1

***************************************

94

S $\quad 6.173750-1.100208-5.699331$

O $5.9343530 .701215-1.921758$

O $7.4154881 .886410-0.035262$

H $\quad 6.9767281 .304772-0.694632$

FE $\quad 6.012445-0.045900-3.409162$

N $4.8003221 .407179-4.281327$

N $7.7319600 .981714-4.044245$

N $7.222592-1.572273-2.666632$

N $4.321095-1.154343-2.944312$

C $3.4362841 .505049-4.133428$

C $2.9707412 .767740-4.707546$

C $4.0870293 .418503-5.197273$

C $5.2314082 .555169-4.912376$

C $7.7312832 .169331-4.743698$

C $9.1114132 .631367-4.913491$

C $9.9329721 .735391-4.277698$

C $9.0391640 .695046-3.707380$

C $8.568586-1.460072-2.423939$

C $9.038279-2.643185-1.677815$

C $7.967316-3.478619-1.519421$

C $\quad 6.807513-2.772418-2.121656$

C $4.308209-2.400061-2.373457$

C $2.932520-2.754879-2.004777$

C $2.150713-1.675201-2.312019$

C $3.018081-0.680636-2.945048$

C $2.6326100 .535784-3.514681$

H $1.5710170 .758114-3.497762$

C $6.5796382 .859332-5.148353$

H $\quad 6.7555553 .814769-5.631822$

C $9.365398-0.391585-2.872418$

H $10.375660-0.406048-2.497396$

C $5.461473-3.150956-2.067286$

H $5.252261-4.118691-1.622451$

C $1.5528813 .275299-4.570195$

H $0.8292862 .480446-4.785885$

H $\quad 1.3736704 .069555-5.302558$

C $1.3478053 .835122-3.143816$

H $2.2173144 .476402-2.939388$

H $1.3803983 .027155-2.405886$

C $0.1135174 .678312-2.840770$

O $-0.2701214 .723511-1.619067$

O $\quad-0.4266285 .397329-3.785407$

$\mathrm{N} \quad-3.096727 \quad 10.089101-3.222581$

$\mathrm{H} \quad-2.4757979 .285332-3.023033$

C $\quad-4.4274219 .988784-2.909862$

N $\quad-5.29050911 .009088-2.987119$

H $\quad-6.27710110 .779932-2.899474$

H $\quad-5.04712712 .026222-3.017418$

N $\quad-4.8785208 .801617-2.492113$

$\mathrm{H} \quad-4.3970637 .901000-2.585427$

H $\quad-5.8650308 .744544-2.235142$

N $\quad-1.3670755 .350483-6.500719$

H $\quad-1.1913885 .124870-7.475440$

H $\quad-0.6720975 .187484-5.776861$
C $\quad-2.6226485 .686331-6.150542$

$\mathrm{N} \quad-3.5454955 .912809-7.088666$

$\mathrm{N} \quad-2.9134285 .804752-4.814221$

H $\quad-2.1420745 .660276-4.139093$

C $\quad-4.1951996 .049814-4.340787$

O $-4.4055696 .158100-3.084865$

C $\quad-5.2134426 .185381-5.344525$

C $\quad-4.8254126 .123857-6.705561$

N $\quad-6.5373766 .327008-4.992876$

H $\quad-6.7603096 .135855-4.021443$

N $\quad-5.7525796 .319546-7.692322$

H $\quad-5.5663795 .907328-8.606451$

C $\quad-7.5763796 .114444-6.032065$

H $\quad-7.6683315 .033718-6.202537$

C $\quad-7.1124516 .775248-7.347741$

H $\quad-7.7754796 .492544-8.167786$

H $\quad-7.1214167 .868269-7.258405$

C $4.6537744 .011624-1.017693$

H $3.6244603 .839292-1.346582$

H $5.1653273 .046328-1.002177$

N $5.2934754 .889251-2.002194$

H $4.7041405 .587763-2.472462$

C $6.6301934 .903420-2.279538$

N $7.4735974 .130263-1.610847$

H $7.4310832 .795512-0.435588$

H $8.4387074 .271476-1.930421$

N $7.0188665 .757398-3.284787$

H $\quad 8.0193785 .893852-3.389607$

H $\quad 6.3774126 .539471-3.513984$

h $4.952333-1.653245-6.025885$

h $4.1720734 .408300-5.645757$

h $\quad 9.3497293 .521829-5.495169$

h $11.0187381 .830459-4.265886$

h $10.059375-2.757910-1.314129$

h $7.863504-4.455613-1.047435$

h $2.662713-3.711254-1.556875$

h $1.094516-1.507245-2.101528$

h $\quad-2.65304910 .804692-3.762072$

h $\quad-8.5470686 .488950-5.707143$

h $4.6491774 .410963-0.003506$

$* * * * * * * * * * * * * * * * * * * * * * * * * * * * * * * * * *$
REACTION 3 SINGLET
REACTANT
TOTAL QM CHARGE: 2
$* * * * * * * * * * * * * * * * * * * * * * * * * * * * * * * * * * *$

95

S $6.095562-1.069208-5.613052$

O $5.9881660 .869861-1.432447$

H $5.4562560 .135113-1.030974$

O $7.2606890 .909770-0.589424$

H $7.8928160 .373884-1.140734$

FE $\quad 6.039119-0.067099-3.515634$

N $4.8413671 .433800-4.097741$

N $7.6816130 .997108-4.016323$

N $7.221900-1.428448-2.611214$

N $4.425021-1.005037-2.741937$

C $3.4705181 .560008-3.912665$

C $2.9826932 .784035-4.544108$

C $4.0738263 .384797-5.135424$

C $5.2240892 .534183-4.852317$

C $7.6768182 .125636-4.819948$

C $9.0458652 .573430-5.063553$

C $9.8890501 .737672-4.377187$

C $9.0188150 .740810-3.716678$

C $8.593013-1.333555-2.386928$

C $9.074007-2.511812-1.659409$

C $8.016686-3.369446-1.531498$

C $\quad 6.850704-2.669957-2.108741$

C $4.396315-2.331983-2.306987$

C $3.022317-2.735014-2.018500$
C $\quad 2.231825-1.642610-2.242981$

C $3.091683-0.583210-2.751451$

C $2.6647430 .629978-3.277851$

H $\quad 1.6029620 .841106-3.259721$

C $6.5354562 .808335-5.226235$

$\mathrm{H} \quad 6.6899323 .711309-5.806448$

C $9.396497-0.297058-2.861373$

H $10.428002-0.347508-2.562318$

C $5.525511-3.102931-2.066200$

H $5.335764-4.106017-1.702477$

C $1.5494593 .269642-4.454487$

H $\quad 0.8549422 .442660-4.643959$

H $\quad 1.3708204 .013676-5.238007$

C $1.2590943 .909788-3.071482$

H $2.0697524 .630014-2.888682$

H $1.3166463 .149508-2.287908$

C $\quad-0.0592854 .662762-2.891355$

O $\quad-0.7047454 .535857-1.802782$

O $\quad-0.4416105 .501996-3.830794$

N $\quad-3.1240549 .894301-2.956348$

H $\quad-2.5151339 .081365-2.774783$

C $-4.4151969 .836353-2.504022$

N $\quad-5.246873 \quad 10.875104-2.446765$

H $\quad-6.21980910 .667301-2.230861$

H $\quad-4.97595711 .886320-2.492197$

N $\quad-4.8460028 .648292-2.040207$

H $\quad-4.4211967 .755236-2.284468$

H $\quad-5.8142908 .619285-1.698232$

$\mathrm{N} \quad-1.4057175 .516469-6.414505$

H $\quad-1.2249335 .303528-7.392280$

H $\quad-0.7127495 .346311-5.680501$

C $\quad-2.6829035 .744315-6.064128$

$\mathrm{N} \quad-3.6032485 .945691-7.012632$

N $\quad-2.9990165 .798292-4.727191$

H $\quad-2.2115375 .687852-4.059726$

C $-4.2981985 .956488-4.265490$

O $\quad-4.5513765 .996107-3.020817$

C $\quad-5.3104896 .085712-5.291649$

C $-4.8915976 .092318-6.655890$

$\mathrm{N} \quad-6.6298116 .145361-4.972753$

$\mathrm{H} \quad-6.8760825 .997325-3.998092$

N $\quad-5.8067556 .284169-7.641251$

H $\quad-5.5812455 .945259-8.578940$

C $\quad-7.6718106 .027251-6.028270$

H $\quad-7.8141884 .956387-6.216607$

C $\quad-7.1807946 .710750-7.319235$

H $\quad-7.8192566 .426012-8.157458$

H $\quad-7.2075447 .802781-7.224720$

C $4.6173923 .937410-0.619798$

H $3.5824473 .793402-0.942019$

H $\quad 5.0400502 .933697-0.537982$

N $5.3062374 .702770-1.672607$

H $4.7441625 .424566-2.153455$

C $6.6140374 .669216-1.971478$

N $7.4700463 .776538-1.431131$

H $7.1831332 .921751-0.968926$

H $8.4420393 .844285-1.696168$

N $7.0947785 .584666-2.831323$

H $8.0974635 .624280-3.004050$

H $6.4891026 .394529-3.075967$

h $4.843113-1.575929-5.894034$

h $4.1281564 .337870-5.661509$

h $9.2601883 .417204-5.719432$

h $10.9727291 .852677-4.399081$

h $10.090933-2.601487-1.277459$

h $7.936783-4.364621-1.094109$

h $2.745983-3.734985-1.684188$

h $1.162072-1.519413-2.074113$

h $\quad-2.73578810 .571949-3.580860$

h $\quad-8.6230246 .454126-5.710409$

h 4.6398294 .4345010 .349977 
REACTION 3 SINGLET PRODUCT

TOTAL QM CHARGE: 2

$* * * * * * * * * * * * * * * * * * * * * * * * * * * * * * * * * * * * *$

95

S $\quad 6.054888-1.042635-5.666444$

O $5.9475040 .820757-1.866591$

H $7.0994730 .980147-0.793585$

O $7.7866981 .362298-0.167293$

H $8.5936960 .805470-0.121175$

FE $5.9882250 .025080-3.329235$

N $4.7845461 .419161-4.176304$

N $7.6343331 .007143-4.013136$

N $7.175572-1.434997-2.615880$

N $4.369093-1.062846-2.863469$

C $3.4189361 .541101-3.990832$

C $2.9330042 .780523-4.584803$

C $4.0301963 .405120-5.143487$

C $5.1753002 .549426-4.873855$

C $7.6357752 .146983-4.800914$

C $\quad 9.0037232 .590409-5.037727$

C $9.8411401 .742897-4.357347$

C $8.9635340 .750611-3.707192$

C $8.529590-1.320361-2.365831$

C $9.017939-2.491831-1.631649$

C $7.967561-3.359170-1.511619$

C $6.806729-2.668521-2.108008$

C $4.349074-2.357492-2.380974$

C $2.980920-2.761119-2.069195$

C $2.189423-1.673359-2.310498$

C $3.049745-0.627941-2.847540$

C $2.6254690 .592064-3.361986$

H $\quad 1.5640620 .805932-3.321887$

C $\quad 6.4976752 .840384-5.196783$

H $\quad 6.6619653 .760796-5.746857$

C $9.331081-0.290570-2.849746$

H $10.360715-0.332308-2.540407$

C $5.486363-3.110060-2.095731$

H $5.297118-4.108047-1.717142$

C $\quad 1.5019863 .266610-4.481953$

H $\quad 0.8036802 .437347-4.645272$

H $\quad 1.3097043 .996714-5.275974$

C $1.2346273 .930552-3.103113$

H $\quad 2.0330524 .672843-2.957533$

H $1.3307363 .186242-2.308112$

C $\quad-0.0973694 .652085-2.908249$

O $-0.7551794 .486895-1.835526$

O $\quad-0.4882495 .508163-3.835560$

N $\quad-3.1254779 .905802-2.957068$

$\mathrm{H} \quad-2.5138529 .095156-2.773181$

C $-4.4148759 .845000-2.502131$

N $\quad-5.25322610 .877783-2.451894$

H $\quad-6.22477110 .665536-2.233454$

$\mathrm{H} \quad-4.98523311 .890150-2.497927$

N $\quad-4.8374118 .656698-2.029078$

H $\quad-4.3937807 .770368-2.253942$

H $\quad-5.8060488 .619370-1.690178$

$\mathrm{N} \quad-1.4332145 .498017-6.404893$

H $\quad-1.2465085 .294591-7.384223$

H $\quad-0.7378525 .342282-5.666387$

C $\quad-2.7053505 .729344-6.056884$

$\mathrm{N} \quad-3.6246035 .933525-7.011754$

$\mathrm{N} \quad-3.0217715 .785032-4.719086$

H $\quad-2.2244715 .678232-4.054511$

C $-4.3207285 .933922-4.259808$

O $\quad-4.5863265 .973047-3.022383$

C $\quad-5.3349766 .052267-5.294001$

C $-4.9118586 .071112-6.662813$

$\mathrm{N}-6.6480446 .083159-4.980878$

H $\quad-6.8973905 .968026-4.001660$

N $\quad-5.8280376 .261924-7.642242$
H $\quad-5.5983385 .943990-8.587101$

C $\quad-7.6938405 .984829-6.036398$

H $\quad-7.8341444 .916025-6.235524$

C $\quad-7.2032946 .686363-7.316082$

H $\quad-7.8401976 .412546-8.158971$

H $\quad-7.2285037 .776811-7.205421$

C $4.7638263 .893455-0.612009$

H $3.7356873 .714361-0.941180$

H $5.2209522 .903569-0.533390$

N $5.4272814 .686303-1.659775$

H $4.8421295 .384109-2.143361$

C $6.7346034 .695133-1.967342$

N $7.6512713 .884592-1.410004$

H $7.4898633 .047673-0.837008$

H $8.6058804 .014875-1.717629$

N $7.1492005 .595455-2.888029$

H $8.1479265 .681916-3.061705$

H $6.5152026 .384042-3.125808$

h $4.827957-1.593814-5.974969$

h $4.0948314 .373752-5.639109$

h $9.2255593 .436652-5.687902$

h $\quad 10.9255581 .851543-4.374667$

h $10.034974-2.574555-1.248422$

h $7.890338-4.354379-1.073826$

h $2.714619-3.748984-1.693331$

h $1.123089-1.543072-2.126044$

h $\quad-2.73791010 .583979-3.581440$

h $\quad-8.6439916 .406465-5.708524$

h 4.7637664 .3900640 .358272

$* * * * * * * * * * * * * * * * * * * * * * * * * * * * * * * * * *$
REACTION 3 TRIPLET
REACTANT
TOTAL QM CHARGE: 2
$* * * * * * * * * * * * * * * * * * * * * * * * * * * * * * * * * *$

95

S $\quad 6.096782-1.168964-5.810803$

O $6.1392380 .973345-1.128871$

H $5.6657280 .206416-0.724098$

O $7.4788500 .961551-0.416767$

H $8.0465440 .414622-1.022543$

FE $6.052001-0.102980-3.582276$

N $4.8598651 .439959-4.117557$

$\mathrm{N} \quad 7.6870761 .002237-4.022907$

N $7.225195-1.416251-2.593966$

N $\quad 4.447589-1.005078-2.766085$

C $3.4880721 .564866-3.934125$

C $3.0003462 .790498-4.550538$

C $4.0933873 .401021-5.134535$

C $5.2413362 .554206-4.857639$

C $7.6883882 .137466-4.823463$

C $9.0526922 .577845-5.061734$

C $9.8939451 .736864-4.374614$

C $9.0269580 .744385-3.720289$

C $8.596572-1.315798-2.370295$

C $9.080390-2.489101-1.643986$

C $8.025302-3.351595-1.511845$

C $6.859933-2.659842-2.083706$

C $4.413316-2.324889-2.308786$

C $3.044300-2.721634-2.021093$

C $2.254972-1.628312-2.256541$

C $3.114027-0.578026-2.772745$

C $2.6847100 .631635-3.301275$

H $1.6227220 .840715-3.281185$

C $6.5517392 .829652-5.225886$

H $\quad 6.7095103 .733750-5.802992$

C $9.401981-0.288536-2.859793$

H $10.434397-0.338929-2.563962$

C $5.539035-3.096021-2.048141$

H $5.346831-4.096345-1.679242$

C $1.5663243 .273731-4.458744$
H $\quad 0.8719622 .447052-4.649723$

H $1.3861284 .018399-5.241255$

C $1.2764923 .911209-3.073332$

H $2.0855594 .632877-2.889297$

H $1.3350213 .149419-2.291195$

C $\quad-0.0436074 .662506-2.892734$

O $\quad-0.6910264 .528501-1.805867$

O $\quad-0.4235965 .504309-3.829356$

N $\quad-3.1240629 .892024-2.957685$

H $\quad-2.5155229 .078909-2.776247$

C $-4.4162139 .833914-2.507504$

N $\quad-5.24640410 .874134-2.448885$

H $\quad-6.22003510 .667165-2.235574$

H $\quad-4.97450411 .884949-2.492948$

N $\quad-4.8492258 .645820-2.046757$

H $\quad-4.4294317 .750845-2.295837$

H $\quad-5.8177198 .619101-1.704701$

$\mathrm{N} \quad-1.3958585 .526220-6.417572$

$\mathrm{H} \quad-1.2169815 .309872-7.394794$

H $\quad-0.7028205 .353987-5.685169$

C $\quad-2.6753175 .749677-6.065843$

$\mathrm{N} \quad-3.5965035 .948900-7.012172$

$\mathrm{N} \quad-2.9910775 .801759-4.729025$

H $\quad-2.2063045 .690704-4.060898$

C $\quad-4.2905575 .960998-4.265897$

O $\quad-4.5390786 .000117-3.018795$

C $\quad-5.3024826 .091686-5.289274$

C $\quad-4.8851766 .096950-6.652169$

$\mathrm{N} \quad-6.6235956 .156668-4.969015$

H $\quad-6.8694116 .006373-3.995035$

$\mathrm{N} \quad-5.8004116 .291195-7.638738$

H $\quad-5.5769195 .947191-8.574779$

C $\quad-7.6640196 .032409-6.024092$

$\mathrm{H} \quad-7.8037114 .961141-6.213271$

C $\quad-7.1744066 .716401-7.315737$

H $\quad-7.8129946 .431707-8.153882$

H $\quad-7.2025817 .808332-7.220232$

C $4.6225693 .954431-0.594743$

H $3.5912863 .804414-0.925863$

H $5.0539342 .954478-0.509212$

N $5.3146324 .725787-1.641528$

H $4.7511845 .443927-2.126732$

C $6.6217354 .692368-1.940981$

N $7.4831333 .815204-1.385562$

H $7.2049562 .987975-0.875048$

H $8.4572783 .889035-1.639621$

N $7.0973965 .590161-2.822973$

H $8.1004605 .633650-2.992942$

H $6.4892026 .394950-3.078311$

h $4.820945-1.650087-6.023184$

h $4.1446854 .355771-5.657877$

h $9.2728583 .422441-5.714613$

h $\quad 10.9777441 .851212-4.393881$

h $10.099833-2.580251-1.269161$

h $7.951397-4.348287-1.076862$

h $2.763809-3.718859-1.682072$

h $1.184705-1.505988-2.090310$

h $\quad-2.73582710 .569595-3.582300$

h $\quad-8.6168936 .456573-5.707576$

h 4.6332164 .4494770 .376279

$* * * * * * * * * * * * * * * * * * * * * * * * * * * * * * * * * * * *$

REACTION 3 TRIPLET PRODUCT TOTAL QM CHARGE: 2

$* * * * * * * * * * * * * * * * * * * * * * * * * * * * * * * * * *$

95

S $\quad 6.060264-1.007893-5.712661$

O $5.9803810 .882203-1.925626$

H $7.1657130 .969475-0.857938$

O $7.8052491 .337322-0.178555$

H $8.6126070 .785310-0.088089$ 
FE $6.0084020 .075543-3.383242$

N $4.7993401 .463741-4.229548$

N $\quad 7.6484381 .054115-4.081771$

N $7.200852-1.380788-2.669537$

N $\quad 4.392587-1.010927-2.901894$

C $3.4358981 .587037-4.031950$

C $2.9446792 .824889-4.626545$

C $4.0356273 .446067-5.199792$

C $5.1842512 .592238-4.934696$

C $7.6456352 .195759-4.865984$

C $9.0122342 .642933-5.106296$

C $9.8532961 .794841-4.432082$

C $8.9798280 .800186-3.779491$

C $8.554838-1.262648-2.424045$

C $9.047242-2.428024-1.681867$

C $7.999614-3.297719-1.556235$

C $6.835969-2.612841-2.154868$

C $4.377025-2.302843-2.412358$

C $3.011046-2.705902-2.087688$

C $2.216219-1.621368-2.331474$

C $3.072739-0.577333-2.879160$

C $2.6455420 .641477-3.393642$

H $1.5850300 .857290-3.344711$

C $6.5045702 .886238-5.260763$

H $\quad 6.6659853 .805948-5.812630$

C $9.353079-0.234134-2.917599$

H $10.384349-0.272032-2.612520$

C $5.516185-3.054743-2.132810$

H $\quad 5.329358-4.050867-1.748280$

C $1.5153383 .312187-4.509397$

H $\quad 0.8138762 .489314-4.691944$

H $\quad 1.3263684 .062527-5.284809$

C $1.2528243 .945082-3.115477$

H $\quad 2.0557434 .679169-2.953854$

$\mathrm{H} \quad 1.3457423 .184205-2.335920$

C $\quad-0.0749404 .671065-2.906686$

O $\quad-0.7237384 .502060-1.828580$

O $\quad-0.4681325 .531635-3.826928$

$\mathrm{N} \quad-3.1321219 .908563-2.953750$

H $\quad-2.5184469 .100424-2.765921$

C $-4.4268819 .843104-2.513090$

N $\quad-5.26398810 .877447-2.464768$

H $\quad-6.23849010 .663856-2.260901$

$\mathrm{H} \quad-4.99299111 .888767-2.502679$

N $\quad-4.8547438 .653412-2.051074$

H $\quad-4.4150957 .765769-2.280965$

H $\quad-5.8279918 .618553-1.721299$

$\mathrm{N} \quad-1.4112385 .515203-6.406081$

H $\quad-1.2240145 .308765-7.384121$

H $\quad-0.7168705 .361659-5.667851$

C $\quad-2.6868995 .738399-6.058215$

$\mathrm{N} \quad-3.6064825 .936625-7.012065$

$\mathrm{N} \quad-3.0040715 .792705-4.721207$

H $\quad-2.2091595 .694740-4.054673$

C $-4.3047775 .932426-4.260922$

O $\quad-4.5647745 .974764-3.021516$

C $\quad-5.3188116 .037620-5.294544$

C $\quad-4.8948976 .067985-6.661006$

$\mathrm{N} \quad-6.6335316 .058288-4.986587$

H $\quad-6.8949836 .000246-4.006558$

$\mathrm{N} \quad-5.8099936 .272446-7.642424$

H $\quad-5.5829495 .951741-8.586574$

C $\quad-7.6714355 .952373-6.046205$

H $\quad-7.7880014 .884346-6.266528$

C $\quad-7.1876316 .683814-7.310757$

H $\quad-7.8208966 .424530-8.161078$

H $\quad-7.2199657 .770878-7.171612$

C $4.7501773 .884407-0.582110$

H $3.7273333 .700050-0.924942$

H $5.2142442 .897651-0.505518$

N $5.4216874 .689568-1.615613$

H $4.8381515 .386233-2.103536$

C $\quad 6.7292294 .699721-1.921274$
N 7.646286 3.889664-1.362946

H $7.4815723 .043838-0.805903$

H $8.6037264 .026822-1.658172$

N $7.1462855 .599596-2.840107$

H $8.1443725 .677944-3.021658$

H $6.5096996 .381212-3.093716$

h $4.832749-1.561829-6.013839$

h $4.0906874 .411153-5.703423$

h $9.2308673 .492063-5.753787$

h $10.9378131 .902139-4.451511$

h $10.063411-2.503574-1.294880$

h $7.924793-4.290613-1.112805$

h $2.750484-3.689874-1.697850$

h $1.148929-1.495454-2.149531$

h $-2.74348910 .585553-3.578748$

h $-8.6306956 .343847-5.707628$

h 4.7324894 .3718250 .392660

$* * * * * * * * * * * * * * * * * * * * * * * * * * * * * * * * * *$
REACTION 3 QUINTET
REACTANT
TOTAL QM CHARGE: 2
$* * * * * * * * * * * * * * * * * * * * * * * * * * * * * * * * * *$

95

S $\quad 6.108023-1.265858-5.885332$

O $6.1681271 .004737-1.205967$

H $5.6759620 .191300-0.929880$

O $7.4695120 .921595-0.434472$

H $8.0547650 .392854-1.037772$

FE $6.082692-0.181863-3.731530$

N $\quad 4.8534371 .460076-4.157339$

N $7.7505271 .017967-4.046253$

N $7.269740-1.452888-2.572117$

$\mathrm{N} \quad 4.436506-1.013875-2.727561$

C $3.4833571 .574555-3.963837$

C $3.0008892 .815220-4.566970$

C $4.1008843 .438009-5.130641$

C $5.2516412 .583918-4.863080$

C $7.7358702 .178103-4.810675$

C $9.1067872 .625657-5.030290$

C $9.9473041 .763767-4.366677$

C $9.0781160 .750609-3.733691$

C $8.643715-1.345191-2.375195$

C $9.127057-2.514703-1.630882$

C $8.066129-3.365460-1.464574$

C $6.892002-2.670884-2.026671$

C $4.413202-2.324628-2.242136$

C $3.034887-2.720572-1.972749$

C $2.244515-1.636248-2.247042$

C $3.109112-0.586985-2.770112$

C $2.6907480 .625634-3.327694$

H $\quad 1.6265070 .829914-3.310702$

C $6.5830252 .865331-5.196409$

H $\quad 6.7425233 .788515-5.744292$

C $9.438833-0.309825-2.881671$

H $\quad 10.472868-0.363890-2.589317$

C 5.552056-3.079781-1.959982

H $\quad 5.356013-4.064429-1.549669$

C $\quad 1.5672503 .299692-4.475943$

H $\quad 0.8728962 .475698-4.679012$

H $1.3903604 .055162-5.249120$

C $1.2688783 .922847-3.085222$

$\mathrm{H} \quad 2.0734804 .647236-2.892974$

H $1.3300393 .153979-2.310263$

C $-0.0557924 .664746-2.899411$

O $\quad-0.7040554 .522903-1.814791$

O $\quad-0.4421395 .511393-3.831572$

N $\quad-3.1254349 .897882-2.956154$

H $\quad-2.5158079 .086132-2.772204$

C $-4.4168809 .838694-2.504921$

N $\quad-5.24989010 .876429-2.449044$
H $\quad-6.22289310 .668054-2.233936$

H $\quad-4.97927911 .887810-2.493232$

$\mathrm{N} \quad-4.8463078 .650125-2.040843$

$\mathrm{H} \quad-4.4193277 .757979-2.283860$

H $\quad-5.8145008 .620100-1.699109$

N $\quad-1.4049805 .518802-6.414040$

$\mathrm{H} \quad-1.2233315 .306015-7.391614$

H $\quad-0.7106505 .353549-5.679859$

C $\quad-2.6819575 .744842-6.063332$

N $\quad-3.6029275 .944014-7.012214$

N $\quad-2.9978275 .797910-4.726156$

H $\quad-2.2088185 .691496-4.059008$

C $-4.2971115 .954292-4.264541$

O $\quad-4.5513805 .994021-3.020324$

C $\quad-5.3098146 .080824-5.290974$

C $\quad-4.8912596 .088994-6.655833$

N $\quad-6.6285526 .134935-4.972199$

H $\quad-6.8738915 .989617-3.996726$

N $\quad-5.8070666 .280011-7.640216$

H $\quad-5.5817605 .944075-8.579069$

C $\quad-7.6712946 .018384-6.026941$

H $\quad-7.8131624 .947785-6.217095$

C $\quad-7.1815426 .704776-7.316568$

H $\quad-7.8201056 .420792-8.154994$

H $\quad-7.2089927 .796555-7.219428$

C $4.6126873 .946770-0.596039$

H $3.5811243 .799935-0.927950$

H $5.0415742 .945524-0.513931$

N $5.3067604 .719972-1.640137$

H $\quad 4.7458125 .439102-2.126071$

C $6.6159394 .687489-1.931549$

N $7.4687313 .802115-1.376006$

H $7.1711852 .953982-0.911533$

H $\quad 8.4436873 .865803-1.628938$

N $7.0989365 .595103-2.798869$

$\mathrm{H} \quad 8.1016185 .631214-2.972818$

H $6.4922646 .400077-3.056113$

h $4.809088-1.699202-6.056583$

h $4.1549714 .398024-5.643976$

h $9.3274053 .477841-5.673078$

h $\quad 11.0315151 .874653-4.382709$

h $10.149837-2.605086-1.265072$

h $7.990525-4.359552-1.023972$

h $2.752478-3.717793-1.635315$

h $1.173352-1.510999-2.088961$

h $\quad-2.73708710 .575351-3.580809$

h $\quad-8.6223606 .444012-5.706970$

h $\quad 4.6249284 .4387720 .376509$

$* * * * * * * * * * * * * * * * * * * * * * * * * * * * * * * * * *$
REACTION 3 QUINTET
PRODUCT
TOTAL QM CHARGE: 2
$* * * * * * * * * * * * * * * * * * * * * * * * * * * * * * * * * * *$

95

S $\quad 6.059912-0.997836-5.701945$

O $5.9869090 .864394-1.928364$

H $7.1687840 .962396-0.857626$

O $7.8048221 .335458-0.177951$

H $8.6148510 .787483-0.087551$

FE $6.0031890 .071058-3.394198$

N $4.7974881 .463339-4.230450$

N $7.6470581 .054719-4.083817$

N 7.199263-1.382906-2.670459

N $4.389990-1.010106-2.900318$

C $3.4335461 .586728-4.033067$

C $2.9434202 .823278-4.627936$

C $4.0348253 .444514-5.202170$

C $5.1829182 .591171-4.937396$

C $7.6448512 .196132-4.869215$

C $9.0111872 .643118-5.107402$ 
C $9.8513901 .794661-4.431759$

C $8.9774710 .800373-3.780444$

C $8.553254-1.262617-2.423277$

C $9.045521-2.427291-1.680560$

C $7.998214-3.297795-1.555579$

C $6.834973-2.614067-2.156229$

C $4.374932-2.303918-2.414002$

C $3.009578-2.706246-2.088267$

C $2.214273-1.621346-2.330898$

C $3.070042-0.576869-2.877586$

C $2.6436220 .641862-3.393048$

H $1.5827920 .856585-3.345049$

C $6.5035422 .885874-5.263089$

H $\quad 6.6644583 .806404-5.813992$

C $9.350689-0.235105-2.918452$

H $\quad 10.382115-0.272541-2.613669$

C $5.514342-3.055746-2.135522$

H $5.328185-4.052460-1.751960$

C $\quad 1.5135453 .309527-4.511884$

H $\quad 0.8126002 .485690-4.691694$

H $\quad 1.3226854 .058035-5.289020$

C $1.2494103 .945258-3.119386$

H $2.0510874 .680747-2.957484$

H $\quad 1.3420643 .186042-2.338314$

C $\quad-0.0790094 .668621-2.910901$

O $-0.7287554 .501086-1.833468$

O $\quad-0.4757495 .527774-3.832824$

N $\quad-3.1304609 .910003-2.956249$

H $\quad-2.5186919 .100922-2.767457$

C $\quad-4.4249389 .845567-2.514696$

$\mathrm{N} \quad-5.26325410 .878759-2.468037$

H $\quad-6.23714710 .664457-2.261278$

H $\quad-4.99312211 .890593-2.505954$

N $\quad-4.8518718 .656241-2.050178$

H $\quad-4.4074987 .769997-2.273323$

H $\quad-5.8242708 .620215-1.718353$

N $\quad-1.4136485 .512015-6.405071$

$\mathrm{H} \quad-1.2254185 .306336-7.383493$

H $\quad-0.7184695 .359717-5.665650$

C $-2.6874895 .735813-6.057761$

$\mathrm{N} \quad-3.6078065 .934529-7.013121$

N $\quad-3.0046675 .792319-4.720394$

H $\quad-2.2059385 .694294-4.055666$

C $-4.3051185 .930202-4.260750$

O $-4.5689835 .970144-3.023664$

C $\quad-5.3201156 .035390-5.296015$

C $\quad-4.8952326 .065692-6.664205$

N $-6.6330096 .053177-4.988782$

$\mathrm{H} \quad-6.8943065 .997475-4.007918$

N $\quad-5.8105776 .269606-7.643606$

H $\quad-5.5831725 .952956-8.589340$

C $\quad-7.6727895 .949647-6.048378$

H $\quad-7.7880904 .881495-6.268219$

C $\quad-7.1881756 .682851-7.310870$

H $\quad-7.8215596 .427622-8.162204$

H $\quad-7.2176657 .769836-7.170192$

C $4.7522123 .883410-0.582912$

H $3.7299003 .698029-0.926754$

H $5.2162122 .896749-0.503509$

N $5.4233994 .687186-1.617199$

H $4.8406735 .383130-2.106500$

C $\quad 6.7314994 .696855-1.921788$

$\mathrm{N} \quad 7.6481303 .887354-1.362093$

H $7.4839633 .040578-0.805601$

H $8.6048634 .021641-1.660709$

N $7.1485885 .597874-2.839061$

H $8.1461915 .674510-3.023594$

H $6.5131056 .380451-3.091626$

h $4.835426-1.556053-6.007517$

h $4.0896964 .409948-5.705153$

h $9.2309063 .492400-5.754325$

h $10.9359141 .901973-4.450671$

h $10.061497-2.502535-1.293006$ h $\quad 7.923625-4.290859-1.112489$

h $2.748990-3.690293-1.698637$

h $1.147013-1.495757-2.148552$

h $\quad-2.74092310 .586707-3.580992$

h $\quad-8.6316526 .341235-5.708808$

h 4.7347474 .3720910 .391230

*****************************************

REACTION 3 SEPTET REACTANT

TOTAL QM CHARGE: 2

$* * * * * * * * * * * * * * * * * * * * * * * * * * * * * * * * * *$

95

S $\quad 6.095394-1.328646-5.941466$

O $5.9803870 .994640-1.201566$

H $5.4850580 .132062-1.290488$

O $7.2356780 .664009-0.430167$

H $7.7938420 .189685-1.097051$

FE $6.107973-0.214897-3.820485$

N $4.8809521 .441149-4.214367$

N $7.7799300 .991182-4.110587$

N $7.293975-1.457257-2.615116$

N $4.465787-1.002572-2.736116$

C $3.5156641 .566219-4.009566$

C $3.0373992 .817415-4.597870$

C $4.1377163 .441215-5.155685$

C $5.2863082 .576650-4.903060$

C $7.7673602 .168177-4.844900$

C $9.1382062 .629784-5.043393$

C $9.9783851 .754489-4.399244$

C $9.1110030 .720900-3.794719$

C $8.672899-1.367125-2.438194$

C $9.151879-2.530384-1.683119$

C $8.084709-3.366667-1.490370$

C $6.910134-2.668777-2.045754$

C $4.435999-2.309837-2.238938$

C $3.057158-2.699577-1.966635$

C $2.267667-1.618693-2.255983$

C $3.132274-0.575335-2.790437$

C $2.7173850 .626914-3.363221$

H $\quad 1.6542510 .836246-3.347910$

C $6.6150862 .861111-5.228803$

H $\quad 6.7771673 .795618-5.755910$

C $9.473906-0.343984-2.959573$

H $\quad 10.509990-0.409287-2.678309$

C $5.572492-3.068772-1.957853$

H $5.376301-4.048085-1.535577$

C $1.6036373 .304573-4.504305$

H $0.9120942 .479990-4.715589$

H $\quad 1.4284444 .063680-5.274381$

C $1.2963883 .917005-3.110702$

H $2.0956764 .644559-2.908192$

H $1.3582933 .144641-2.339341$

C $\quad-0.0344384 .649712-2.927687$

O $-0.6880184 .499765-1.847568$

O $\quad-0.4243915 .497427-3.858555$

N $\quad-3.1217099 .892227-2.959645$

H $\quad-2.5129999 .078727-2.780997$

C $\quad-4.4117949 .835605-2.504383$

N $\quad-5.24306110 .874223-2.444380$

H $\quad-6.21566010 .666720-2.226311$

H $\quad-4.97293711 .885893-2.491034$

N $\quad-4.8428128 .646977-2.040835$

H $\quad-4.4125467 .756001-2.279447$

H $\quad-5.8103488 .616771-1.697501$

N $\quad-1.4028485 .500994-6.421725$

H $\quad-1.2228875 .293298-7.401202$

$\mathrm{H} \quad-0.7062075 .336356-5.687994$

C $\quad-2.6765385 .730796-6.068247$

$\mathrm{N} \quad-3.5993765 .936008-7.015724$

$\mathrm{N} \quad-2.9893585 .783153-4.729813$

H $\quad-2.1969795 .674214-4.066069$
C $\quad-4.2867995 .940807-4.265278$

O $-4.5410535 .980489-3.022412$

C $\quad-5.3028136 .068348-5.291354$

C $-4.8861256 .079906-6.658705$

N $\quad-6.6192326 .116610-4.970911$

$\mathrm{H} \quad-6.8635905 .974745-3.994440$

N $\quad-5.8040496 .271587-7.639226$

$\mathrm{H} \quad-5.5789055 .941489-8.580558$

C $\quad-7.6657636 .006545-6.024029$

H $\quad-7.8088304 .936364-6.215080$

C $\quad-7.1781556 .695972-7.312515$

H $\quad-7.8179166 .413824-8.150560$

$\mathrm{H} \quad-7.2052867 .787591-7.213399$

C $4.5544393 .916074-0.558307$

H $3.5208643 .788658-0.892192$

H $4.9604642 .905961-0.471618$

N $5.2668354 .675576-1.601631$

H $4.7274815 .416873-2.078534$

C $\quad 6.5765844 .614585-1.887974$

N $7.3946323 .684606-1.351486$

H $7.0509102 .840206-0.913730$

H $8.3701543 .704144-1.605851$

N $7.0901525 .533493-2.724296$

H $8.0953045 .558431-2.890717$

H $6.5006556 .351666-2.979983$

h $4.782660-1.728503-6.087030$

h $4.1997214 .412288-5.646845$

h $9.3621173 .510300-5.645574$

h $11.0622261 .869807-4.406812$

h $10.173945-2.619744-1.315067$

h $8.005517-4.352027-1.031173$

h $2.776101-3.690863-1.611072$

h $1.194743-1.494005-2.109853$

h $\quad-2.73351810 .571398-3.582547$

h $\quad-8.6156956 .432633-5.701313$

h 4.5758204 .4116580 .412263

REACTION 3 SEPTET PRODUCT

TOTAL QM CHARGE: 2

***************************************

95

S $\quad 6.090508-1.001375-5.692377$

O $5.9891410 .852845-1.934988$

H $7.1599280 .952619-0.864880$

O $7.8041321 .324027-0.190035$

H $8.6135560 .774540-0.103993$

FE $5.9952000 .062386-3.420669$

N $4.7497031 .496746-4.271601$

N $7.6999911 .065661-4.127805$

N $7.230892-1.454917-2.688766$

N $4.328413-1.062222-2.910347$

C $3.3884701 .588295-4.088932$

C $2.9067922 .845078-4.660311$

C $4.0102103 .489231-5.195767$

C $5.1610842 .632389-4.934619$

C $7.6709652 .232580-4.867177$

C $9.0421022 .686881-5.096977$

C $9.8885221 .810469-4.462277$

C $9.0193330 .783707-3.840579$

C $8.586933-1.332544-2.482548$

C $9.074730-2.485816-1.708180$

C $8.016190-3.334872-1.529881$

C $6.842529-2.651660-2.125088$

C $4.336581-2.327400-2.373089$

C $2.965484-2.727084-2.047052$

C $2.162557-1.660326-2.348571$

C $3.015365-0.623558-2.927992$

C $2.6073200 .601683-3.471372$

H $1.5420950 .804484-3.444040$

C $6.5060752 .920104-5.229722$ 
H $\quad 6.6667013 .857085-5.754900$

C $9.376042-0.298619-3.007387$

H $10.413788-0.362097-2.727544$

C 5.501107-3.055705-2.066962

H $5.312824-4.029741-1.628085$

C $1.4781883 .333034-4.542523$

H $\quad 0.7756602 .512348-4.730732$

H $\quad 1.2886994 .093076-5.308523$

C $1.2205073 .948609-3.139268$

H $2.0264914 .677997-2.970029$

H $\quad 1.3146603 .176866-2.370471$

C $\quad-0.1034804 .673617-2.915607$

O $-0.7480614 .498602-1.836751$

O $-0.5016395 .543449-3.827325$

N $\quad-3.1311849 .915428-2.956980$

H $\quad-2.5186299 .107413-2.764758$

C $-4.4258659 .849089-2.516878$

$\mathrm{N} \quad-5.26726210 .879794-2.476178$

H $\quad-6.24044210 .664096-2.268203$

$\mathrm{H} \quad-4.99766011 .891726-2.512470$

N $\quad-4.8499098 .659990-2.049116$

H $\quad-4.3995537 .775473-2.265697$

H $\quad-5.8230068 .620983-1.720054$

N $\quad-1.4280175 .513601-6.403847$

H $\quad-1.2377375 .309555-7.382182$

H $\quad-0.7332265 .366072-5.663247$

C $\quad-2.7015515 .739570-6.058026$

N $\quad-3.6203195 .936617-7.015526$

$\mathrm{N} \quad-3.0202255 .798132-4.721335$

H $\quad-2.2216145 .703111-4.054989$

C $-4.3216525 .931319-4.263161$

O $\quad-4.5876205 .970752-3.026966$

C $\quad-5.3357336 .031760-5.300613$

C $\quad-4.9083486 .064398-6.668644$

$\mathrm{N} \quad-6.6486276 .041536-4.995962$

H $\quad-6.9121205 .988055-4.015465$

N $\quad-5.8230206 .264610-7.648630$

$\mathrm{H} \quad-5.5924595 .951751-8.594942$

C $\quad-7.6864705 .940539-6.057913$

$\mathrm{H} \quad-7.8009964 .873317-6.281765$

C $\quad-7.2005146 .677903-7.317301$

H $\quad-7.8325696 .425698-8.170421$

H $\quad-7.2291587 .764400-7.172697$

C $4.7589643 .877676-0.586734$

H $3.7363593 .691171-0.929201$

H $5.2240492 .891400-0.506106$

N $5.4294474 .679591-1.622857$

H $4.8463835 .375628-2.111390$

C $6.7374974 .689762-1.926717$

N $7.6543233 .879876-1.367488$

H $7.4908203 .027945-0.817706$

H $8.6108424 .014569-1.666840$

N $7.1548965 .593335-2.841723$

H $8.1526605 .670109-3.026248$

H $\quad 6.5197946 .377333-3.090544$

h $4.870313-1.565978-6.003332$

h $4.0692304 .461751-5.684425$

h $9.2561493 .553553-5.722382$

h $\quad 10.9726701 .921871-4.479035$

h $10.090847-2.550918-1.319164$

h $7.937924-4.316261-1.062104$

h $2.708597-3.704641-1.639060$

h $1.097633-1.526157-2.158832$

h $\quad-2.74116010 .592102-3.581451$

h $\quad-8.6460296 .330598-5.718550$

h 4.7428784 .3692130 .385993

****************************************

REACTANT

REACTION 4 SINGLET

TOTAL QM CHARGE: 0

96
Energy $=0.00 \mathrm{kcal} / \mathrm{mol}$

S $\quad 6.139000-1.039000-5.644000$

O $5.8560000 .637000-1.552000$

O $6.9160001 .130000-0.828000$

FE $6.0190000 .001000-3.470000$

$\mathrm{N} \quad 4.8430001 .500000-4.140000$

N $7.6890001 .051000-4.007000$

N $7.189000-1.426000-2.677000$

N $4.362000-0.979000-2.873000$

C $3.4730001 .633000-3.980000$

C $3.0110002 .870000-4.599000$

C $4.1170003 .473000-5.159000$

C $5.2510002 .608000-4.869000$

C $7.7010002 .202000-4.774000$

C $9.0720002 .651000-4.985000$

C $9.9050001 .768000-4.342000$

C $9.0200000 .753000-3.726000$

C $8.549000-1.349000-2.454000$

C $9.003000-2.535000-1.720000$

C $7.924000-3.359000-1.563000$

C $\quad 6.777000-2.641000-2.160000$

C $4.325000-2.281000-2.410000$

C $2.947000-2.661000-2.097000$

C $2.175000-1.554000-2.316000$

C $3.054000-0.519000-2.850000$

C $2.6470000 .707000-3.363000$

H $\quad 1.5880000 .935000-3.344000$

C $6.5700002 .891000-5.201000$

H $\quad 6.7360003 .805000-5.762000$

C $9.377000-0.338000-2.935000$

H $\quad 10.417000-0.434000-2.670000$

C $5.448000-3.057000-2.142000$

H $5.243000-4.051000-1.758000$

C $1.6060003 .411000-4.444000$

H $\quad 0.8590002 .630000-4.633000$

H $\quad 1.4270004 .200000-5.183000$

C $1.4450003 .995000-3.025000$

H $2.3250004 .632000-2.853000$

H $1.4950003 .190000-2.283000$

C $\quad 0.2290004 .848000-2.683000$

O $-0.0630004 .934000-1.432000$

O $\quad-0.3930005 .514000-3.606000$

N $\quad-3.1800009 .968000-3.037000$

$\mathrm{H} \quad-2.6720009 .106000-2.815000$

C $-4.53800010 .002000-2.811000$

N $\quad-5.21800011 .162000-2.732000$

H $\quad-6.23000011 .158000-2.861000$

H $\quad-4.80900012 .114000-2.820000$

N $\quad-5.1590008 .853000-2.587000$

H $\quad-4.7770007 .894000-2.762000$

H $\quad-6.1530008 .883000-2.301000$

N $\quad-1.3600005 .238000-6.282000$

H $\quad-1.1420004 .974000-7.234000$

H $\quad-0.7010005 .103000-5.522000$

C $\quad-2.6490005 .565000-5.996000$

N $\quad-3.5370005 .700000-6.968000$

$\mathrm{N} \quad-2.9930005 .772000-4.685000$

H $\quad-2.2710005 .681000-3.967000$

C $\quad-4.3020006 .063000-4.290000$

O $-4.5320006 .278000-3.026000$

C $\quad-5.2620006 .139000-5.313000$

C $-4.8350005 .948000-6.640000$

N $\quad-6.6290006 .383000-5.030000$

H $\quad-6.8810006 .141000-4.077000$

$\mathrm{N} \quad-5.7270006 .015000-7.665000$

H $\quad-5.3980005 .773000-8.592000$

C $\quad-7.5930005 .955000-6.078000$

H $\quad-7.6430004 .858000-6.139000$

C $\quad-7.0960006 .481000-7.442000$

H $\quad-7.7200006 .098000-8.254000$

H $\quad-7.1360007 .577000-7.468000$

C $4.6820003 .830000-0.672000$

H $3.6460003 .705000-0.998000$
H $5.1140002 .829000-0.644000$

N $5.3600004 .666000-1.675000$

H $4.7950005 .435000-2.079000$

C $\quad 6.6360004 .617000-2.084000$

N $7.5100003 .670000-1.696000$

H $7.2250002 .745000-1.354000$

H $8.4330003 .713000-2.107000$

$\mathrm{N} \quad 7.0690005 .590000-2.911000$

H $8.0570005 .614000-3.145000$

H $6.4800006 .432000-3.070000$

O 6.7760000 .6580001 .912000

H 6.7100000 .8960000 .950000

H $\quad 6.690000-0.3130001 .979000$

h $4.892878-1.560368-5.926313$

h $4.1957754 .448023-5.639823$

h $9.3011673 .521395-5.599785$

h $10.9899021 .869906-4.367868$

h $10.022147-2.664345-1.355753$

h $7.812837-4.333634-1.087817$

h $2.654112-3.651630-1.749242$

h $1.113316-1.408423-2.116750$

h $\quad-2.66039810 .643222-3.560509$

h $\quad-8.5868646 .333884-5.839784$

h 4.6991254 .2673940 .326228

****************************************

REACTION 4 SINGLET PRODUCT TOTAL QM CHARGE: 0

***************************************

96

Energy $=0.00 \mathrm{kcal} / \mathrm{mol}$

S $6.128000-1.038000-5.632000$

O $5.8600000 .915000-1.745000$

O $7.1470000 .962000-0.891000$

FE $6.0210000 .053000-3.454000$

N $4.8340001 .518000-4.186000$

N $7.7120001 .061000-4.033000$

N $7.203000-1.377000-2.641000$

N $4.361000-0.931000-2.870000$

C $3.4670001 .652000-4.036000$

C $3.0120002 .899000-4.646000$

C $4.1250003 .506000-5.183000$

C $5.2560002 .632000-4.889000$

C $7.7140002 .223000-4.781000$

C $9.0850002 .676000-4.996000$

C $9.9230001 .785000-4.373000$

C $9.0440000 .759000-3.766000$

C $8.581000-1.337000-2.469000$

C $9.026000-2.523000-1.732000$

C $7.931000-3.319000-1.534000$

C $\quad 6.785000-2.585000-2.106000$

C $4.331000-2.220000-2.374000$

C $2.951000-2.610000-2.077000$

C $2.172000-1.516000-2.331000$

C $3.050000-0.484000-2.875000$

C $2.6390000 .728000-3.418000$

H $1.5790000 .950000-3.409000$

C $6.5810002 .917000-5.198000$

H $6.7520003 .840000-5.743000$

C $9.410000-0.335000-2.978000$

H $10.453000-0.441000-2.732000$

C $5.452000-2.986000-2.074000$

H $5.242000-3.972000-1.673000$

C $1.6080003 .442000-4.489000$

H $\quad 0.8590002 .664000-4.679000$

H $1.4310004 .235000-5.225000$

C $1.4530004 .021000-3.066000$

H $2.3270004 .669000-2.902000$

H $\quad 1.5220003 .214000-2.329000$

C $\quad 0.2310004 .856000-2.707000$

O $-0.0600004 .922000-1.454000$

O $\quad-0.3990005 .530000-3.619000$ 


\begin{abstract}
N $\quad-3.1800009 .970000-3.037000$
$\mathrm{H} \quad-2.6730009 .107000-2.816000$

C $-4.53800010 .003000-2.811000$

$\mathrm{N} \quad-5.21800011 .163000-2.732000$

$\mathrm{H} \quad-6.23000011 .158000-2.862000$

$\mathrm{H} \quad-4.81000012 .115000-2.821000$

N $\quad-5.1580008 .854000-2.588000$

H $\quad-4.7760007 .895000-2.763000$

H $\quad-6.1520008 .884000-2.301000$

N $\quad-1.3650005 .243000-6.292000$

H $\quad-1.1500004 .978000-7.244000$

H $\quad-0.7030005 .116000-5.533000$

C $-2.6540005 .568000-6.002000$

$\mathrm{N} \quad-3.5450005 .701000-6.972000$

$\mathrm{N} \quad-2.9950005 .774000-4.691000$

$\mathrm{H} \quad-2.2710005 .685000-3.974000$

C $-4.3040006 .064000-4.292000$

O $\quad-4.5310006 .279000-3.028000$

C $\quad-5.2660006 .137000-5.313000$

C $\quad-4.8430005 .947000-6.640000$

$\mathrm{N} \quad-6.6320006 .381000-5.025000$

H $\quad-6.8810006 .139000-4.071000$

$\mathrm{N} \quad-5.7370006 .011000-7.663000$

H $\quad-5.4090005 .773000-8.591000$

C $\quad-7.5980005 .949000-6.070000$

H $\quad-7.6460004 .852000-6.130000$

C $\quad-7.1060006 .475000-7.436000$

H $\quad-7.7320006 .090000-8.246000$

H $\quad-7.1490007 .571000-7.463000$

C $4.6150003 .781000-0.692000$

$\mathrm{H} \quad 3.5730003 .702000-1.018000$

H $5.0060002 .760000-0.716000$

N $5.3120004 .623000-1.679000$

H $4.7630005 .405000-2.081000$

C $6.5960004 .573000-2.056000$

$\mathrm{N} 7.4550003 .615000-1.658000$

H $7.1610002 .681000-1.364000$

H $8.3890003 .665000-2.042000$

$\mathrm{N} \quad 7.0580005 .559000-2.852000$

$\mathrm{H} \quad 8.0490005 .578000-3.072000$

H $\quad 6.4760006 .405000-3.019000$

O $\quad 6.9820001 .3550001 .744000$

H $\quad 6.8880001 .1490000 .751000$

H $7.6460000 .172000-1.207000$

h $4.883077-1.561459-5.915730$

h $4.2112034 .486182-5.651912$

h $9.3099213 .554368-5.600935$

h $11.0076431 .889232-4.400430$

h $10.049137-2.664379-1.383762$

h $7.813566-4.285877-1.044690$

h $2.661172-3.601048-1.727872$

h $1.108357-1.374034-2.139750$

h $-2.66077210 .644280-3.562091$

h $\quad-8.5921106 .325857-5.829604$

h 4.6470944 .1968000 .315049
\end{abstract}

$* * * * * * * * * * * * * * * * * * * * * * * * * * * * * * * * * *$ REACTION 5 SINGLET

\section{REACTANT}

TOTAL QM CHARGE: 1

$* * * * * * * * * * * * * * * * * * * * * * * * * * * * * * * * * * * *$

97

Energy $=0.00 \mathrm{kcal} / \mathrm{mol}$

S $\quad 6.219000-1.038000-5.460000$

O $6.0270000 .629000-1.205000$

O $7.0060001 .431000-0.615000$

H 7.6610002 .0240002 .134000

FE $6.093000-0.015000-3.323000$

N $4.9170001 .464000-3.995000$

$\mathrm{N} \quad 7.7410001 .052000-3.828000$

N $7.264000-1.403000-2.474000$

N $4.461000-1.000000-2.703000$
C $3.5420001 .578000-3.854000$

C $3.0590002 .767000-4.540000$

C $\quad 4.1580003 .367000-5.120000$

C $5.3070002 .539000-4.784000$

C $7.7580002 .156000-4.665000$

C $9.1280002 .615000-4.855000$

C $9.9480001 .799000-4.116000$

C $9.0620000 .811000-3.466000$

C $8.615000-1.292000-2.196000$

C $9.090000-2.504000-1.521000$

C $8.037000-3.376000-1.455000$

C $6.880000-2.660000-2.031000$

C $4.429000-2.313000-2.265000$

C $\quad 3.055000-2.705000-1.953000$

C $2.275000-1.600000-2.154000$

C $3.145000-0.555000-2.679000$

C $2.7280000 .659000-3.209000$

H $\quad 1.6660000 .872000-3.205000$

C $\quad 6.6250002 .813000-5.133000$

H $\quad 6.7860003 .701000-5.735000$

C $9.414000-0.228000-2.603000$

H $\quad 10.428000-0.246000-2.239000$

C $5.557000-3.094000-2.030000$

H $\quad 5.357000-4.102000-1.683000$

C $1.6340003 .265000-4.442000$

H $\quad 0.9210002 .454000-4.628000$

H $\quad 1.4510004 .023000-5.211000$

C $1.4110003 .885000-3.047000$

H $\quad 2.2720004 .546000-2.870000$

H $\quad 1.4450003 .109000-2.276000$

C $0.1720004 .734000-2.784000$

O $\quad-0.2410004 .776000-1.567000$

O $\quad-0.3240005 .461000-3.736000$

$\mathrm{N} \quad-3.09700010 .071000-3.201000$

H $\quad-2.4830009 .266000-2.994000$

C $\quad-4.4440009 .966000-2.945000$

N $\quad-5.29200011 .003000-3.044000$

$\mathrm{H} \quad-6.28200010 .782000-2.997000$

$\mathrm{H} \quad-5.03700012 .015000-3.046000$

$\mathrm{N} \quad-4.9190008 .784000-2.559000$

$\mathrm{H} \quad-4.4700007 .851000-2.686000$

$\mathrm{H} \quad-5.9220008 .749000-2.350000$

$\mathrm{N} \quad-1.2730005 .377000-6.540000$

$\mathrm{H} \quad-1.1200005 .116000-7.508000$

H $\quad-0.5800005 .194000-5.827000$

C $-2.5390005 .721000-6.181000$

N $\quad-3.4660005 .937000-7.102000$

$\mathrm{N} \quad-2.8200005 .858000-4.848000$

H $\quad-2.0660005 .710000-4.167000$

C $-4.0980006 .140000-4.370000$

O $\quad-4.2580006 .262000-3.091000$

C $\quad-5.1120006 .300000-5.347000$

C $-4.7420006 .191000-6.693000$

$\mathrm{N} \quad-6.4570006 .538000-4.980000$

H $\quad-6.6720006 .225000-4.039000$

N $\quad-5.6630006 .401000-7.701000$

H $\quad-5.5080005 .897000-8.572000$

C $\quad-7.4700006 .207000-6.018000$

H $\quad-7.5170005 .119000-6.167000$

C $\quad-7.0260006 .842000-7.356000$

H $\quad-7.6890006 .537000-8.169000$

H $\quad-7.0510007 .936000-7.284000$

C $4.5560003 .960000-0.986000$

H $3.5290003 .851000-1.334000$

$\mathrm{H} \quad 4.9870002 .957000-1.001000$

$\mathrm{N} \quad 5.2560004 .839000-1.934000$

H $4.6920005 .597000-2.354000$

C $6.5480004 .778000-2.295000$

N $7.4020003 .845000-1.836000$

H $7.1190002 .950000-1.415000$

H $8.3340003 .859000-2.229000$

$\mathrm{N} \quad 7.0140005 .719000-3.140000$

H $8.0110005 .755000-3.328000$
H $\quad 6.4100006 .538000-3.346000$

O 7.3180001 .1970001 .710000

H 7.1320001 .2130000 .727000

H 7.4410000 .3510002 .194000

h $4.991799-1.579122-5.784825$

h $4.2115844 .323332-5.640215$

h $9.3801223 .436051-5.526101$

h $11.0329611 .903338-4.122276$

h $10.110863-2.633645-1.161698$

h $7.949334-4.390419-1.065983$

h $2.774414-3.703382-1.617484$

h $1.209433-1.463408-1.969674$

h $-2.64902810 .771975-3.755911$

h $-8.4585086 .551534-5.714331$

h 4.5495544 .3503150 .031683

****************************************
REACTION 5 SINGLET
INTERMEDIATE
TOTAL QM CHARGE: 1
$* * * * * * * * * * * * * * * * * * * * * * * * * * * * * * * * *$

97

Energy $=0.00 \mathrm{kcal} / \mathrm{mol}$

S $\quad 6.215000-1.041000-5.461000$

O $6.0870000 .862000-1.517000$

O $7.3480000 .821000-0.650000$

H $7.681000-0.098000-0.783000$

FE $6.159000-0.014000-3.230000$

N $4.9690001 .447000-3.975000$

$\mathrm{N} \quad 7.8290000 .980000-3.836000$

$\mathrm{N} \quad 7.326000-1.481000-2.486000$

N $4.501000-1.026000-2.691000$

C $3.6000001 .566000-3.860000$

C $3.1250002 .774000-4.525000$

C $4.2390003 .390000-5.057000$

C $5.3790002 .553000-4.709000$

C $7.8430002 .135000-4.592000$

C $9.2130002 .577000-4.813000$

C $\quad 10.0430001 .696000-4.165000$

C $9.1590000 .691000-3.545000$

C $8.693000-1.425000-2.269000$

C $9.151000-2.628000-1.575000$

C $8.071000-3.461000-1.453000$

C $6.923000-2.719000-2.007000$

C $4.458000-2.324000-2.228000$

C $3.081000-2.712000-1.923000$

C $2.304000-1.611000-2.152000$

C $3.185000-0.577000-2.682000$

C $2.7800000 .632000-3.231000$

H $\quad 1.7190000 .848000-3.235000$

C $6.7070002 .833000-5.006000$

H $\quad 6.8810003 .747000-5.565000$

C $9.512000-0.389000-2.731000$

H $\quad 10.540000-0.448000-2.415000$

C $5.585000-3.111000-1.974000$

H $5.373000-4.110000-1.611000$

C $1.6950003 .266000-4.452000$

H $\quad 0.9900002 .457000-4.674000$

$\mathrm{H} \quad 1.5320004 .037000-5.212000$

C $1.4330003 .862000-3.052000$

$\mathrm{H} \quad 2.2880004 .515000-2.834000$

H $1.4390003 .072000-2.294000$

C $\quad 0.1890004 .710000-2.803000$

O $\quad-0.2280004 .758000-1.589000$

O $\quad-0.3100005 .425000-3.763000$

N $\quad-3.09800010 .073000-3.212000$

H $\quad-2.4820009 .269000-3.009000$

C $-4.4410009 .970000-2.941000$

N $\quad-5.29100011 .005000-3.030000$

H $\quad-6.28100010 .784000-2.972000$

$\mathrm{H} \quad-5.03800012 .018000-3.038000$

N $\quad-4.9130008 .787000-2.551000$ 


\begin{abstract}
H $\quad-4.4590007 .860000-2.673000$
H $\quad-5.9120008 .748000-2.331000$

$\mathrm{N} \quad-1.2820005 .363000-6.535000$

$\mathrm{H} \quad-1.1270005 .112000-7.505000$

$\mathrm{H} \quad-0.5890005 .180000-5.821000$

C $\quad-2.5450005 .708000-6.176000$

N $\quad-3.4710005 .928000-7.098000$

$\mathrm{N} \quad-2.8270005 .840000-4.842000$

H $\quad-2.0720005 .689000-4.163000$

C $\quad-4.1050006 .118000-4.362000$

O $\quad-4.2710006 .239000-3.088000$

C $\quad-5.1220006 .275000-5.344000$

C $-4.7480006 .177000-6.693000$

N $\quad-6.4610006 .498000-4.981000$

$\mathrm{H} \quad-6.6800006 .210000-4.032000$

N $\quad-5.6700006 .389000-7.697000$

H $\quad-5.5110005 .901000-8.577000$

C $\quad-7.4800006 .193000-6.019000$

$\mathrm{H} \quad-7.5380005 .106000-6.175000$

C $\quad-7.0310006 .835000-7.351000$

$\mathrm{H} \quad-7.6940006 .537000-8.167000$

H $\quad-7.0510007 .928000-7.273000$

C $4.2750003 .871000-0.763000$

H $3.2330003 .829000-1.080000$

H $4.6380002 .840000-0.767000$

N $5.0080004 .689000-1.750000$

H $\quad 4.5010005 .483000-2.175000$

C $6.3230004 .602000-2.018000$

N $7.0570003 .565000-1.580000$

H $6.6310002 .656000-1.383000$

H $8.0470003 .556000-1.765000$

N $6.9020005 .583000-2.732000$

$\mathrm{H} \quad 7.9100005 .573000-2.883000$

H $6.3520006 .437000-2.957000$

O $\quad 6.7510000 .7530002 .159000$

H 6.8970000 .8150001 .191000

H $\quad 7.145000-0.0610002 .536000$

h $4.993359-1.594153-5.786518$

h $4.3097614 .356823-5.555309$

h $9.4516113 .441597-5.432358$

h $\quad 11.1289741 .785516-4.191698$

h $10.163596-2.745951-1.189249$

h $7.969121-4.458630-1.025892$

h $2.793784-3.706496-1.581606$

h $1.236854-1.468616-1.981726$

h $-2.650788 \quad 10.779673-3.760258$

h $-8.4657896 .542955-5.712701$

h 4.3237464 .2796040 .246323
\end{abstract}
$* * * * * * * * * * * * * * * * * * * * * * * * * * * * * * * * * * *$
REACTION 5 SINGLET PRODUCT TOTAL QM CHARGE: 1

****************************************

97

Energy $=0.00 \mathrm{kcal} / \mathrm{mol}$

S $6.199000-1.144000-5.581000$

O $6.0010000 .480000-1.420000$

O $7.2450001 .160000-0.750000$

H $7.9530000 .590000-1.159000$

FE $6.098000-0.160000-3.481000$

N $4.9240001 .381000-4.049000$

$\mathrm{N} \quad 7.7550000 .936000-3.930000$

N $7.271000-1.534000-2.608000$

N $4.454000-1.086000-2.784000$

C $3.5430001 .505000-3.911000$

C $3.0760002 .726000-4.546000$

C $\quad 4.1830003 .336000-5.101000$

C $5.3220002 .486000-4.801000$

C $7.7710002 .079000-4.717000$

C $9.1410002 .541000-4.899000$

C $9.9640001 .698000-4.196000$

C $9.0820000 .687000-3.582000$
C $\quad 8.622000-1.423000-2.315000$

C $9.082000-2.607000-1.585000$

C $8.021000-3.466000-1.493000$

C $\quad 6.874000-2.763000-2.105000$

C $4.420000-2.390000-2.311000$

C $3.046000-2.763000-1.976000$

C $2.268000-1.660000-2.193000$

C $3.132000-0.634000-2.757000$

C $2.7200000 .579000-3.289000$

H $\quad 1.6600000 .801000-3.278000$

C $6.6400002 .763000-5.144000$

H $\quad 6.8030003 .672000-5.713000$

C $9.429000-0.356000-2.717000$

H $\quad 10.439000-0.375000-2.346000$

C $\quad 5.543000-3.173000-2.067000$

H $\quad 5.337000-4.165000-1.679000$

C $\quad 1.6570003 .239000-4.432000$

H $\quad 0.9340002 .439000-4.630000$

H $\quad 1.4800004 .010000-5.189000$

C $1.4380003 .844000-3.029000$

H $\quad 2.3040004 .494000-2.835000$

H $\quad 1.4490003 .058000-2.266000$

C $\quad 0.2030004 .707000-2.772000$

O $\quad-0.2090004 .757000-1.556000$

O $\quad-0.2870005 .429000-3.729000$

N $\quad-3.09700010 .072000-3.203000$

H $\quad-2.4820009 .269000-2.996000$

C $-4.4440009 .967000-2.943000$

N $\quad-5.29200011 .003000-3.039000$

$\mathrm{H} \quad-6.28200010 .783000-2.989000$

$\mathrm{H} \quad-5.03800012 .016000-3.043000$

N $\quad-4.9170008 .784000-2.558000$

$\mathrm{H} \quad-4.4650007 .853000-2.684000$

H $\quad-5.9190008 .747000-2.346000$

$\mathrm{N} \quad-1.2600005 .359000-6.539000$

H $-1.1100005 .103000-7.508000$

H $\quad-0.5650005 .173000-5.829000$

C $\quad-2.5230005 .709000-6.176000$

N $\quad-3.4510005 .928000-7.095000$

$\mathrm{N} \quad-2.8020005 .847000-4.842000$

H $\quad-2.0510005 .693000-4.161000$

C $-4.0780006 .135000-4.361000$

O $\quad-4.2340006 .260000-3.083000$

C $\quad-5.0940006 .296000-5.338000$

C $-4.7260006 .186000-6.685000$

N $\quad-6.4370006 .538000-4.970000$

H $\quad-6.6530006 .231000-4.027000$

$\mathrm{N} \quad-5.6480006 .399000-7.692000$

H $\quad-5.4950005 .894000-8.563000$

C $\quad-7.4530006 .209000-6.007000$

H $\quad-7.5030005 .121000-6.154000$

C $\quad-7.0100006 .842000-7.345000$

$\mathrm{H} \quad-7.6730006 .537000-8.158000$

H $\quad-7.0330007 .936000-7.274000$

C $4.5500003 .975000-0.847000$

$\mathrm{H} \quad 3.5330003 .808000-1.204000$

H $\quad 5.0260002 .992000-0.798000$

$\mathrm{N} \quad 5.2340004 .823000-1.831000$

H $4.6530005 .553000-2.280000$

C $6.5390004 .814000-2.147000$

N $7.4220003 .930000-1.639000$

H $7.1730003 .055000-1.191000$

H $8.3860004 .029000-1.930000$

N $6.9920005 .742000-3.006000$

H $\quad 7.9870005 .780000-3.207000$

H $\quad 6.3770006 .550000-3.240000$

O 6.8410001 .0540001 .993000

H 7.0800000 .8730001 .038000

H $\quad 5.3000001 .166000-1.299000$

h $4.951144-1.647854-5.886504$

h $4.2477304 .306949-5.592055$

h $9.3889513 .389331-5.536904$

h $11.0480751 .810957-4.186587$ h $\quad 10.091345-2.711492-1.187042$

h $7.926917-4.460924-1.057868$

h $2.760146-3.754209-1.624065$

h $1.205905-1.511835-1.997891$

h $-2.64988410 .775263-3.755703$

h $\quad-8.4409266 .554739-5.702806$

h 4.5162554 .3899930 .160327

\section{REACTION 6 SINGLET \\ REACTANT}

TOTAL QM CHARGE: 2

***************************************

98

Energy $=0.00 \mathrm{kcal} / \mathrm{mol}$

S $6.178000-0.938000-5.359000$

O $\quad 6.1230000 .894000-1.257000$

H $\quad 5.7390000 .0240001 .568000$

O $7.4530000 .890000-0.439000$

H $8.0370000 .216000-0.865000$

FE $6.1450000 .031000-3.153000$

N $4.9450001 .517000-3.820000$

N $7.8070001 .049000-3.739000$

$\mathrm{N} \quad 7.326000-1.417000-2.429000$

N $\quad 4.503000-0.986000-2.605000$

C $3.5670001 .619000-3.705000$

C $3.0840002 .817000-4.386000$

C $4.1930003 .427000-4.936000$

C $5.3410002 .603000-4.582000$

C $7.8030002 .177000-4.543000$

C $\quad 9.1700002 .598000-4.820000$

C $\quad 10.0150001 .738000-4.162000$

C $9.1440000 .760000-3.483000$

C $8.693000-1.334000-2.186000$

C $9.157000-2.542000-1.500000$

C $8.096000-3.405000-1.437000$

C $\quad 6.943000-2.676000-2.006000$

C $4.478000-2.323000-2.234000$

C $3.102000-2.743000-1.968000$

C $2.311000-1.643000-2.153000$

C $3.181000-0.562000-2.602000$

C $\quad 2.7570000 .667000-3.105000$

H $\quad 1.6920000 .863000-3.119000$

C $6.6620002 .871000-4.936000$

H $\quad 6.8190003 .766000-5.528000$

C $9.513000-0.305000-2.653000$

H $\quad 10.550000-0.386000-2.372000$

C $5.612000-3.106000-2.025000$

H $5.418000-4.133000-1.735000$

C $\quad 1.6390003 .277000-4.378000$

$\mathrm{H} \quad 0.9680002 .438000-4.591000$

H $\quad 1.4850004 .010000-5.177000$

C $\quad 1.2830003 .923000-3.017000$

H $\quad 2.0810004 .649000-2.810000$

$\mathrm{H} \quad 1.3200003 .165000-2.230000$

C $\quad-0.0420004 .674000-2.872000$

O $\quad-0.6970004 .554000-1.785000$

O $\quad-0.4080005 .506000-3.818000$

$\mathrm{N} \quad-3.1230009 .888000-2.959000$

$\mathrm{H} \quad-2.5140009 .075000-2.778000$

C $-4.4190009 .827000-2.520000$

N $\quad-5.24600010 .871000-2.458000$

H $\quad-6.22200010 .666000-2.255000$

$\mathrm{H} \quad-4.97100011 .881000-2.497000$

$\mathrm{N} \quad-4.8590008 .638000-2.071000$

$\mathrm{H} \quad-4.4570007 .736000-2.339000$

H $\quad-5.8290008 .618000-1.730000$

N $-1.3800005 .542000-6.429000$

H $\quad-1.2090005 .315000-7.404000$

H $\quad-0.6890005 .356000-5.702000$

C $\quad-2.6650005 .763000-6.074000$

$\mathrm{N} \quad-3.5870005 .958000-7.014000$ 


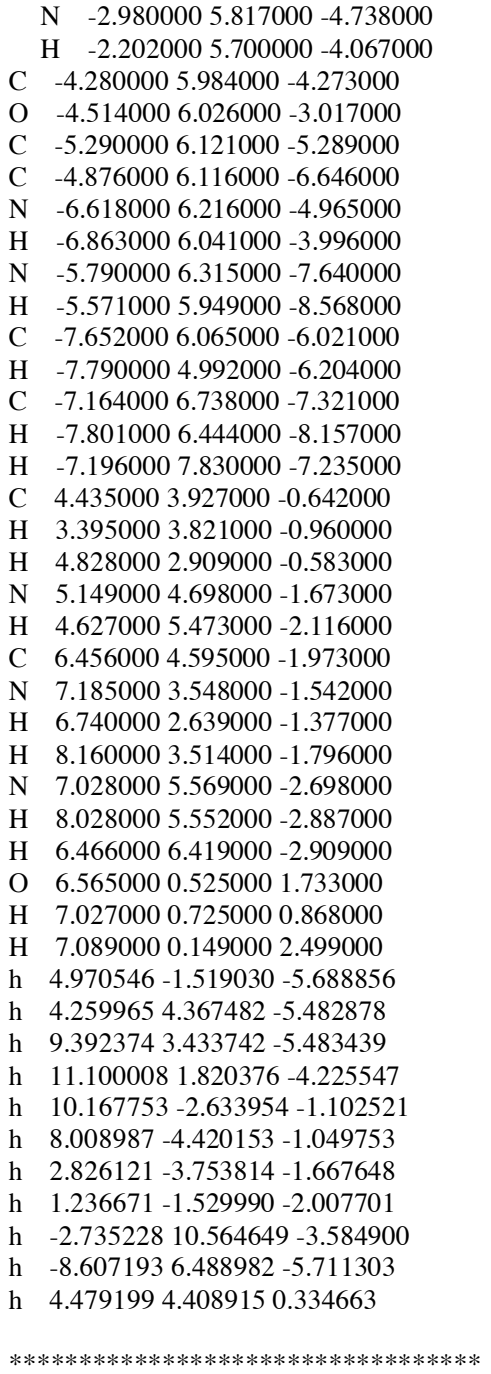

REACTION 6 SINGLET PRODUCT TOTAL QM CHARGE: 2

$* * * * * * * * * * * * * * * * * * * * * * * * * * * * * * * * * *$

98

Energy $=0.00 \mathrm{kcal} / \mathrm{mol}$

S $\quad 6.096000-1.074000-5.613000$

O $5.9940000 .881000-1.443000$

H $\quad 5.4770000 .174000-0.980000$

O $7.2870000 .966000-0.633000$

H $7.9090000 .405000-1.172000$

FE $6.036000-0.067000-3.517000$

N $4.8390001 .432000-4.106000$

N $7.6800000 .996000-4.024000$

N $7.221000-1.425000-2.611000$

$\mathrm{N} \quad 4.424000-1.005000-2.748000$

C $3.4680001 .559000-3.920000$

C $\quad 2.9820002 .784000-4.549000$

C $4.0730003 .386000-5.139000$

C $5.2230002 .533000-4.860000$

C $7.6760002 .125000-4.828000$

C $9.0450002 .575000-5.068000$

C $9.8880001 .739000-4.382000$

C $9.0180000 .741000-3.723000$

C $8.592000-1.329000-2.387000$

C $9.073000-2.507000-1.658000$

C $8.017000-3.365000-1.531000$

C $6.851000-2.667000-2.109000$

C $4.397000-2.330000-2.310000$

C $3.023000-2.734000-2.019000$
C $\quad 2.232000-1.643000-2.245000$

C $3.091000-0.584000-2.757000$

C $2.6630000 .628000-3.285000$

H $\quad 1.6010000 .838000-3.266000$

C $6.5350002 .807000-5.234000$

H $\quad 6.6890003 .709000-5.815000$

C $9.396000-0.294000-2.865000$

H $\quad 10.428000-0.345000-2.568000$

C $\quad 5.526000-3.101000-2.068000$

H $\quad 5.337000-4.104000-1.704000$

C $\quad 1.5500003 .272000-4.456000$

H $\quad 0.8540002 .446000-4.645000$

H $\quad 1.3700004 .016000-5.240000$

C $\quad 1.2610003 .912000-3.073000$

H $\quad 2.0710004 .634000-2.892000$

$\mathrm{H} \quad 1.3210003 .152000-2.289000$

C $\quad-0.0580004 .663000-2.892000$

O $\quad-0.7050004 .534000-1.804000$

O $\quad-0.4410005 .503000-3.831000$

N $\quad-3.1240009 .895000-2.956000$

$\mathrm{H} \quad-2.5150009 .082000-2.774000$

C $-4.4150009 .837000-2.504000$

N $\quad-5.24700010 .875000-2.447000$

H $\quad-6.22000010 .667000-2.231000$

$\mathrm{H} \quad-4.97600011 .886000-2.492000$

$\mathrm{N} \quad-4.8450008 .648000-2.039000$

$\mathrm{H} \quad-4.4200007 .756000-2.283000$

H $\quad-5.8140008 .619000-1.697000$

$\mathrm{N} \quad-1.4050005 .515000-6.414000$

H $\quad-1.2240005 .302000-7.392000$

$\mathrm{H} \quad-0.7110005 .346000-5.680000$

C $-2.6820005 .743000-6.064000$

$\mathrm{N} \quad-3.6020005 .944000-7.012000$

N $\quad-2.9980005 .797000-4.727000$

$\mathrm{H} \quad-2.2110005 .687000-4.059000$

C $\quad-4.2970005 .956000-4.265000$

O $\quad-4.5510005 .996000-3.020000$

C $\quad-5.3090006 .085000-5.291000$

C $-4.8910006 .091000-6.656000$

$\mathrm{N} \quad-6.6290006 .143000-4.973000$

H $\quad-6.8750005 .997000-3.998000$

N $\quad-5.8060006 .283000-7.641000$

H $\quad-5.5800005 .944000-8.579000$

C $\quad-7.6710006 .025000-6.028000$

H $\quad-7.8130004 .955000-6.217000$

C $-7.1800006 .709000-7.319000$

H $\quad-7.8180006 .424000-8.157000$

H $\quad-7.2070007 .801000-7.225000$

C $4.6250003 .947000-0.628000$

H $3.5910003 .799000-0.951000$

H $\quad 5.0520002 .945000-0.548000$

N $5.3100004 .718000-1.680000$

H $\quad 4.7450005 .440000-2.156000$

C $\quad 6.6170004 .687000-1.984000$

N $7.4780003 .797000-1.449000$

H $7.1980002 .936000-0.995000$

H $8.4490003 .872000-1.718000$

N $7.0930005 .603000-2.846000$

H $8.0950005 .643000-3.022000$

H $6.4850006 .413000-3.087000$

O $\quad 6.6600000 .7490002 .130000$

H 7.0420000 .7830001 .232000

H 7.0710000 .0280002 .662000

h $4.843696-1.579943-5.896024$

h $4.1280784 .340187-5.662984$

h $9.2608693 .420793-5.720764$

h $10.9717311 .854190-4.400023$

h $10.090396-2.595920-1.277125$

h $7.937900-4.360406-1.093992$

h $2.748545-3.733165-1.680753$

h $1.162485-1.519340-2.074967$

h $-2.73592610 .572828-3.580435$

h $\quad-8.6220296 .452223-5.710050$ h $\quad 4.6449804 .4400740 .343877$ 


\section{CHARMM Parameter file used}

Standard CHARMM22 parameter file was used, with the following additions/modifications:

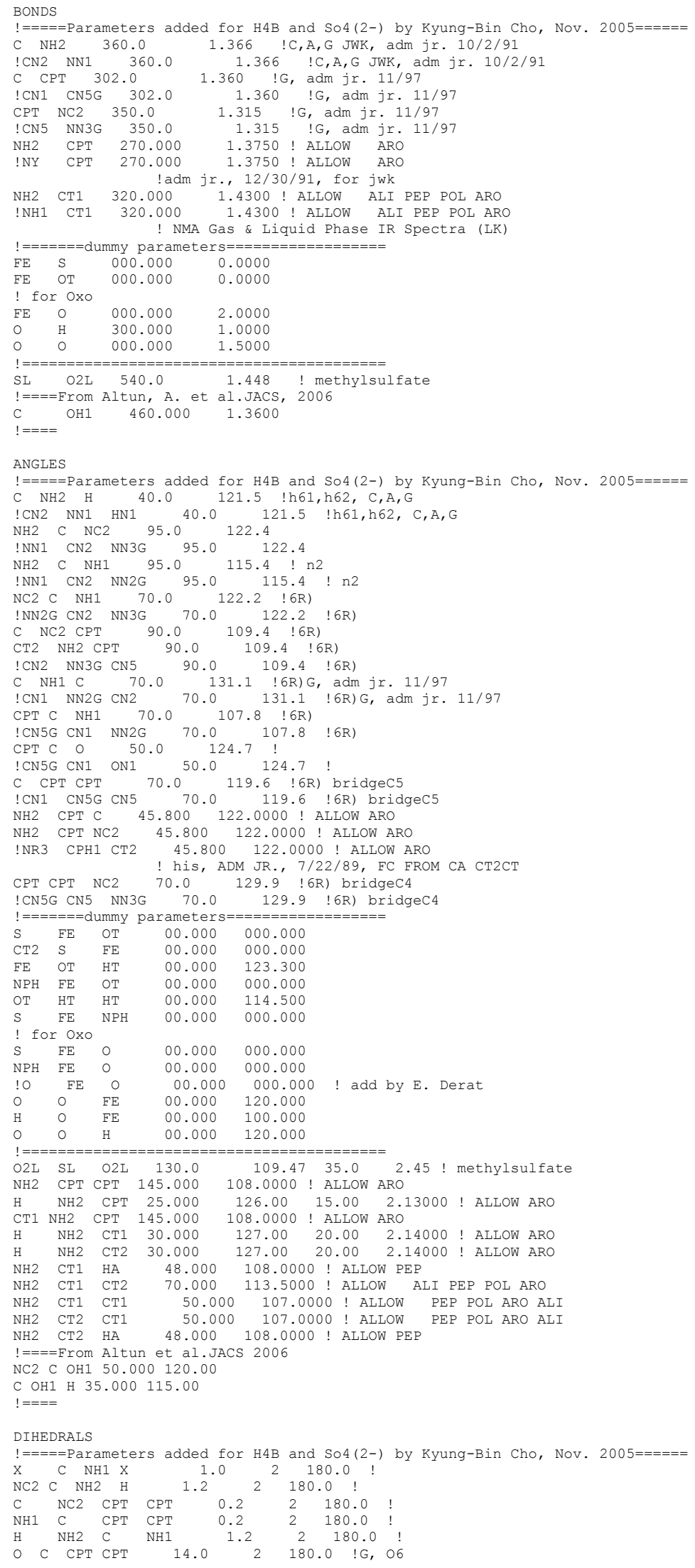




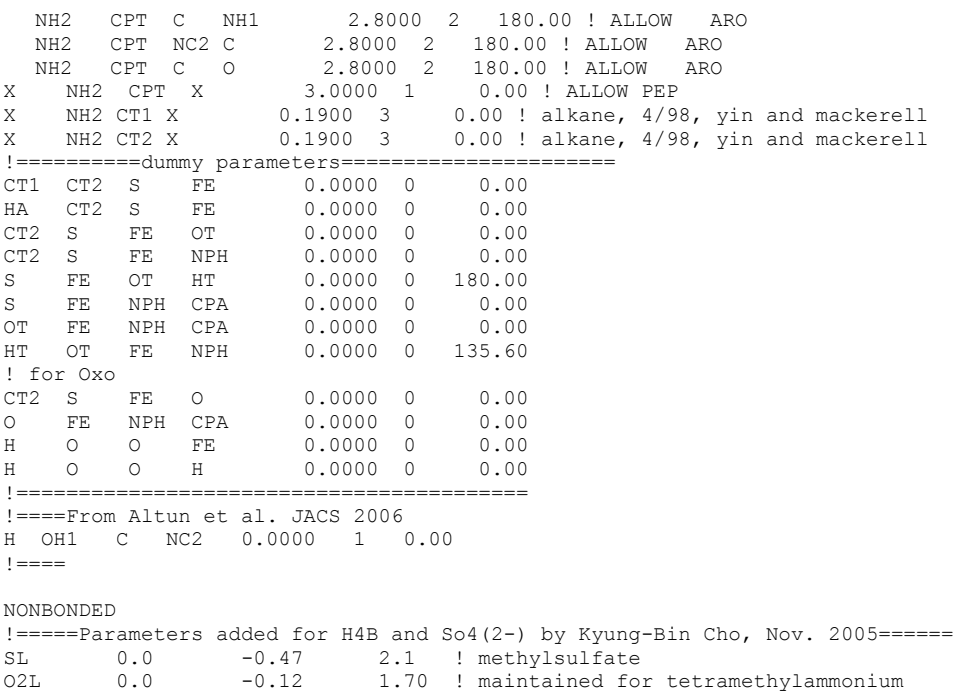




\section{Topology files used}

General CHARMM22 topology files are used, in addition to these:

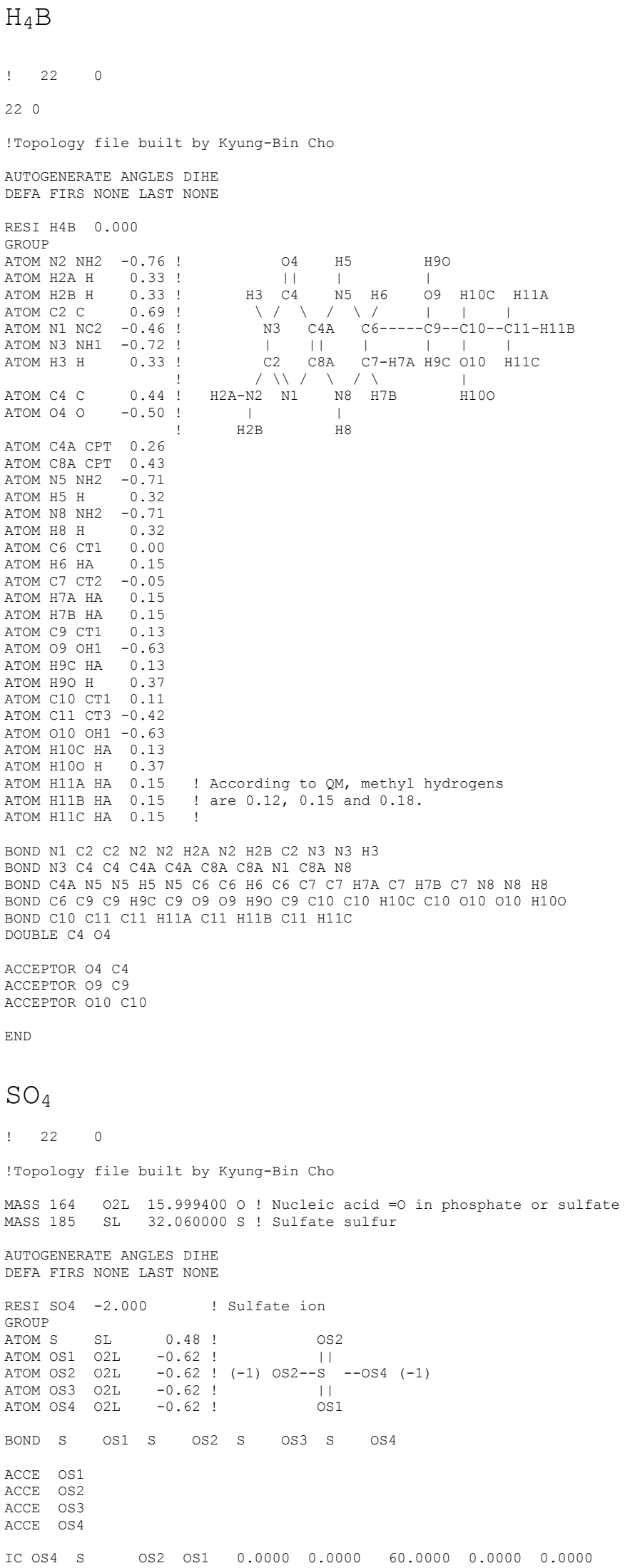

BOND N1 C2 C2 N2 N2 H2A N2 H2B C2 $\mathrm{N} 3$ N3 H3

BOND N3 C4 C4 C4A C4A C8A C8A N1 C8A N8

BOND C4A N5 N5 H5 N5 C6 C6 6 H6 C6 C7 C7 H7A C7 H7B C7 $\mathrm{N} 8$ N8 H8

BOND C6 C9 C9 H9C C9 09 o9 H9o C9 C10 C10 H10C C10 010 o10 H100

BOND C10 C11 C11 H11A C11 H11B C11 H11C

DOUBLE C4 04

ACCEPTOR O4 C4

ACCEPTOR O9 C9

ACCEPTOR 010 C10

END

$\mathrm{SO}_{4}$ 


\begin{tabular}{|c|c|c|c|c|c|c|c|c|c|c|c|}
\hline & IC 0 & & $\mathrm{~S}$ & & & OS 3 & 0.00 & 0.00 & -60.00 & 0.00 & 0.0000 \\
\hline & IC $O$ & & $\mathrm{~s}$ & & & OS 4 & 0.00 & 0.00 & 60.00 & 0.00 & 0.0000 \\
\hline & IC 0 & & $\mathrm{~s}$ & & & OS2 & 0.00 & 0.00 & 60.00 & 0.00 & 0.0000 \\
\hline IC & os1 & $\mathrm{s}$ & & OS 4 & os2 & & 0.0000 & 0.0000 & 180.0000 & 0.0000 & 0.0000 \\
\hline IC & os1 & $\mathrm{s}$ & & OS2 & os 3 & & 0.0000 & 0.0000 & 180.0000 & 0.0000 & 0.0000 \\
\hline IC & os1 & $\mathrm{S}$ & & os 3 & os2 & & 0.0000 & 0.0000 & 180.0000 & 0.0000 & 0.0000 \\
\hline IC & OS 4 & S & & os2 & os1 & & 0.0000 & 0.0000 & 60.0000 & 0.0000 & 0.0000 \\
\hline IC & OS 4 & $\mathrm{~S}$ & & os 3 & OS1 & & 0.0000 & 0.0000 & 60.0000 & 0.0000 & 0.0000 \\
\hline IC & os 3 & $\mathrm{~s}$ & & os 2 & OS1 & & 0.0000 & 0.0000 & 60.0000 & 0.0000 & 0.0000 \\
\hline
\end{tabular}

END

\section{Heme- $\mathrm{O}_{2}$}

271 ! this is the version number

!Topology file for 1DZ9-- Conpound I of P450cam viewd by Schilchting et.al. ! Further modified to describe Fe-OO by Kyung-Bin Cho.

MASS 93 POT $39.10200 \mathrm{~K}$ ! Potassium Ion check masses

DECL $-C A$

DECL -C

DECL $-O$

DECL $+\mathrm{N}$

$\mathrm{DECL}+\mathrm{HN}$

DECL +CA

DEFA FIRS NTER LAST CTER

AUTO ANGLES DIHE

RESI POT $\quad 1.00$ ! Potassium Ion

GROUP

PATCHING FIRS NONE LAST NONE

!naming the hem+cys toxo group with a total charge of -2.00

RESI HEM -3.00

GROUP

ATOM N NH1

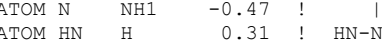

$\begin{array}{llllrr}\text { ATOM HN } & \text { H } & 0.31 & \text { HN }-\mathrm{N} & \\ \text { ATOM CA } & \text { CT1 } & 0.07 & ! & \text { HB } \\ \text { ATOM HAY } & \text { HB } & 0.09 & ! & \text { | }\end{array}$

GROUP HAY-CA--CB--(SG) move definition of HA-->HAY

$-0.18 \div \quad|\quad|$

\begin{tabular}{l|lll}
0.09 & 1 & HB2
\end{tabular}

GROUP

$0.09 ! \quad \mathrm{O}=\mathrm{C}$

$\begin{array}{lll}\text { ATOM C } & \text { C } & 0.51\end{array}$

АТОМ $\bigcirc \quad \circ \quad-0.51$

!adding the heme

GROUP

ATOM SG S -0.07 ! the charge on the HGI atom included in the SG GROUP ATOM OF1 $0 \quad-0.66$ ! the oxo group

ATOM OF2 O -0.66

ATOM $\mathrm{FE} \quad \mathrm{FE}-0.63$

ATOM NA $\mathrm{NPH}-0.63$

ATOM NB NPH -0.38

ATOM NC NPH -0.38

ATOM ND NPH -0.38

$\begin{array}{lll}\text { ATOM C1A CPA } & 0.20\end{array}$

ATOM C2A CPB $\quad-0.04$

АTOM CЗА $\mathrm{CPB}-0.04$

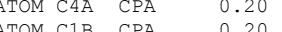

ATOM C2B CPB

ATOM $\mathrm{C} 3 \mathrm{~B} \quad \mathrm{CPB}$

ATOM $C 4 B$ CPA

ATOM C4B CPA

ATOM C2C $\mathrm{CPB}$

ATOM C $C$ C $\mathrm{CPB}$

ATOM C4C CPA

ATOM C1D CPA

ATOM C2D CPB

ATOM C3D CPB

ATOM C4D CPA

GROUP

ATOM CHA CPM

ATOM HA HA

GROUP

ATOM CHB CPM

ATOM HB HA

GROUP

ATOM CHC CPM

ATOM HC HA

GROUP

ATOM CHD CPM -0.10

ATOM HD HA 0.10

GROUP

ATOM CMA CT3

ATOM HMA2 HA

ATOM HMA 3 HA $\quad 0.09$

GROUP

ATOM CAA CT2 -0.18

$\begin{array}{lll}\text { ATOM HAA1 HA } & 0.09 \\ \text { ATOM HAA2 } & \text { HA } & 0.09\end{array}$ 


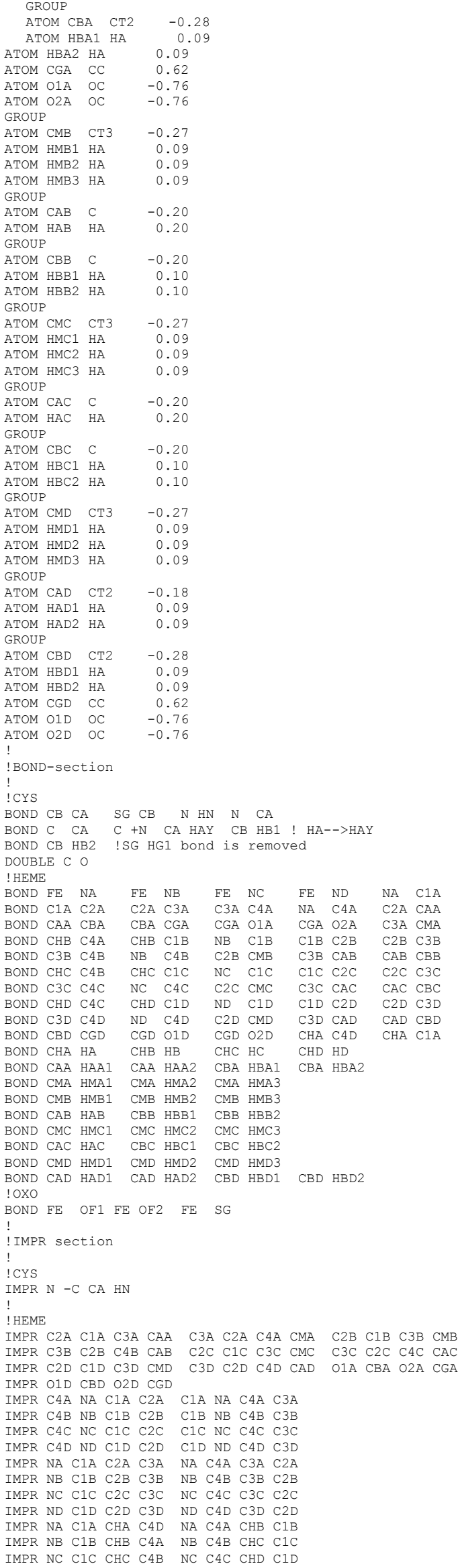




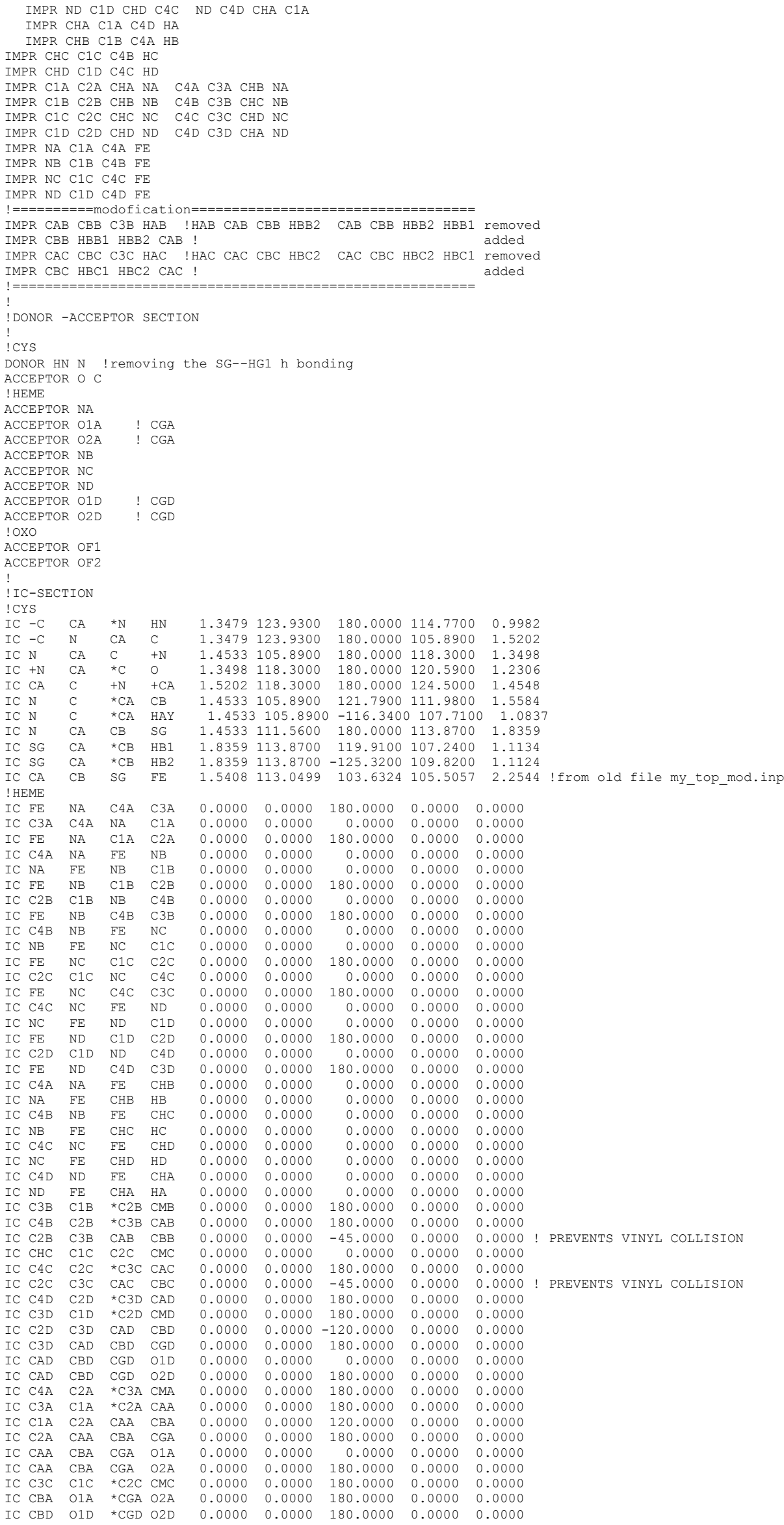




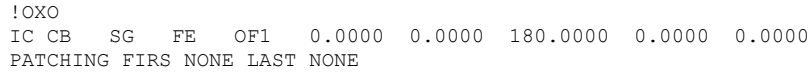

Heme-OOH

271 !this is the version number

!Topology file for 1DZ9-- Conpound I of P450cam viewd by Schilchting et.al. ! Further modified to describe Fe-OOH by Kyung-Bin Cho.

MASS 93 РОт $39.10200 \mathrm{~K}$ ! Potassium Ion check masses

DECL $-C A$

DECL $-C$

DECL $-O$

DECL +N

DECL +HN

DECL +CA

DEFA FIRS NTER LAST CTER

AUTO ANGLES DIHE

RESI POT

1.00 ! Potassium Ion

GROUP

ATOM POT POT 1.00

PATCHING FIRS NONE LAST NONE

!naming the hem+cys toxo group with a total charge of -2.00

RESI HEH -2.00

GROUP

ATOM N NH1 -0.47 !

$0.37 !+1$

$\begin{array}{llrrrr}\text { ATOM HN } & \text { H } & 0.31 & ! & \text { HN-N } & \\ \text { ATOM } & \text { CA } & \text { CT1 } & 0.07 & ! & \text { I }\end{array}$

GROUP

ATOM $\mathrm{CB} \quad \mathrm{CT} 2$

ATOM HB1 HA

ATOM HB2 HA

GROUP

$0.09 ! \begin{aligned} & \mid \\ & !\end{aligned}$

$-0.18$

0.09

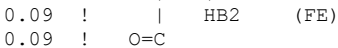

$\begin{array}{lll}\text { ATOM C } & \mathrm{C} & 0.51\end{array}$

ATOM $0 \quad 0 \quad-0.51$

ladding the heme

ATOM SG S

ATOM OF1 0

ATOM OF2 0

ATOM HF2 H

ATOM FE FE

ATOM NA NPH

ATOM NB NPH

ATOM NC NPH

ATOM C1A CPA

ATOM C2A CPB

ATOM C3A CPB

ATOM C4A CPA

ATOM C1B CPA

ATOM C2B CPB

ATOM C4B CPA

ATOM C1C CPA

ATOM C2C CPB

ATOM C3C CPB

ATOM C4C CPA

ATOM C1D CPA

ATOM C2D CPB

ATOM C3D CPB

ATOM C4D CPA

GROUP

ATOM CHA CPM

ATOM HA HA

GROUP

ATOM CHB CPM

ATOM HB HA

GROUP

ATOM CHC CPM

-0.07 ! the charge on the HG1 atom included in the SG GROUP -0.32 ! the oxo group

$-0.32 !$

$0.32 !$

$0.63 !$

$-0.38 !$

$-0.38$

$-0.38 !$

$-0.38$

-0.04 !

$-0.04$

$-0.04$

0.20 :

\begin{tabular}{llccc|r}
-0.04 & ! HMA1 1 & I & I & | & /HMD1 \\
-0.04 & ! HMA2-CMA--C3A & NA & OF2 & ND & C2D--CMD-HMD2
\end{tabular}

0.20 !

$-0.04 !$

-0.04 !

$0.20 !$

$0.20 !$

-0.04 !HMB $1 \backslash$

0.20 ! HMB $3 /$

$-0.10 !$

0.10 !

$-0.10 !$

$0.10 !$

CPM

ATOM

ATOM CHD CPM

$-0.10 !$

0.10 !

$-0.10$

GROUP

GROUP

ATOM HMA1 HA

ATOM HMA2 HA 0.09

ATOM HMA3 HA

GROUP

ATOM CAA CT2

ATOM HAA1 HA

ATOM HAA2 HA

GROUP

ATOM HBA1 HA

ATOM HBA2 HA

ATOM CGA CC

ATOM O1A OC

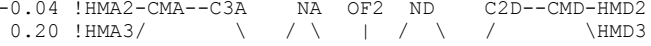

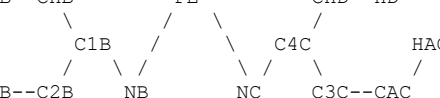

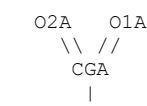

।
HBA1--CBA--HBA2 HA HBD1--CBD--HBD2

HAA1--CAA-HAA2 CHA HAD1--CAD--HAD2

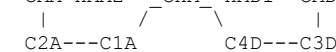

C2A--C1A HF2

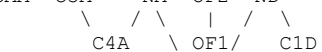

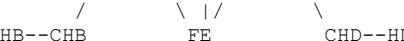

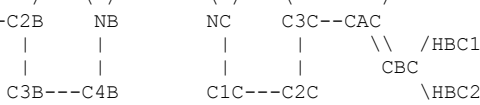

$\mathrm{CAB} \quad-\frac{1}{1} \quad \mathrm{CMC}--\mathrm{HMC} 3$

$\mathrm{CBB} \quad \mathrm{HAB}$

HBB1 HBB2

0.09

$-0.18$

0.09

0.09

$-0.28$

0.09

0.09

0.62
-0.76 


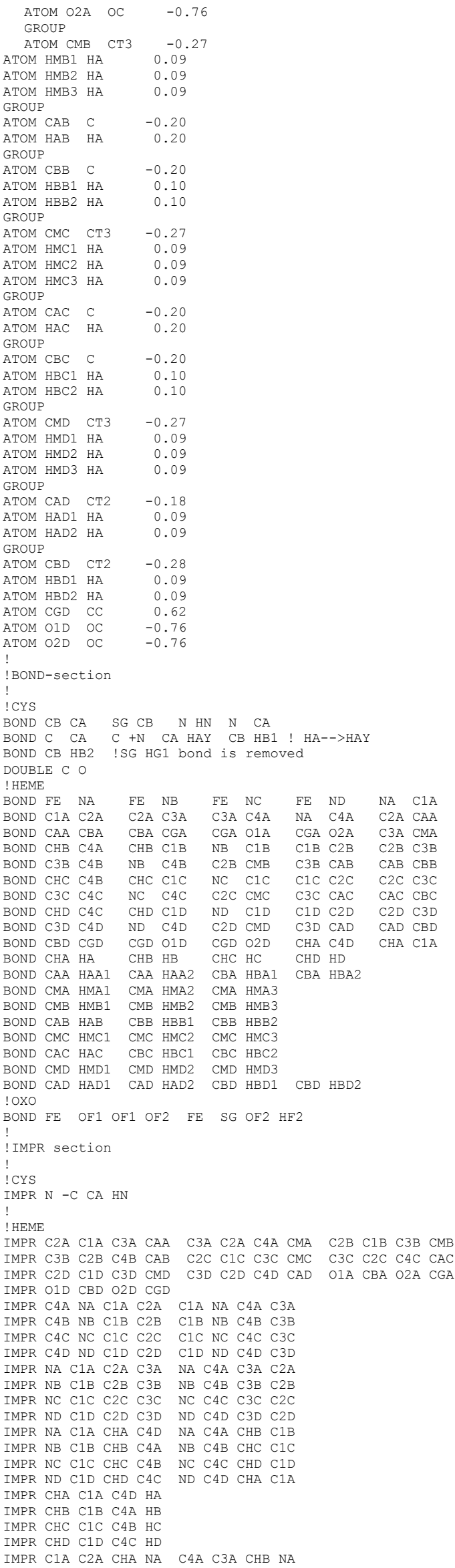


IMPR C1B C2B CHB NB C4B C3B CHC NB

IMPR C1C C2C CHC NC C4C C3C CHD NC

IMPR C1D C2D CHD ND C4D C3D CHA ND

IMPR NA C1A C4A FE

IMPR NB C1B C4B FE

$\begin{array}{lllll}\text { IMPR NC } & \text { C1C } & \text { C4C } & \text { FE } \\ \text { IMPR } & \text { ND } & \text { C1D } & \text { C4D } & \text { FE }\end{array}$

$!=========$ modof ication $=================================$
IMPR CAB CBB C3B HAB ! HAB CAB CBB HBB2 CAB CBB HBB2 HBB1

IMPR CBB HBB1 HBB2 CAB !

IMPR CAC CBC C3C HAC ! HAC CAC CBC HBC2 CAC CBC HBC2 HBC1 removed

IMPR CBC HBC1 HBC2 CAC ! added

$!=$

IDONOR -ACCEPTOR SECTION

DONOR HN N !removing the SG--HG1 h bonding

ACCEPTOR O $\mathrm{C}$

! HEME

ACCEPTOR NA

ACCEPTOR O1A ! CGA

ACCEPTOR O2A ! CGA

ACCEPTOR NB

ACCEPTOR NC

ACCEPTOR ND

ACCEPTOR O1D ! CGD

ACCEPTOR O2D ! CGD

!OXO

ACCEPTOR OF1

ACCEPTOR OF 2

DONOR HF2 NB

IC-SECTION

!CYS

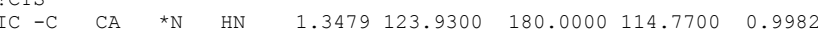

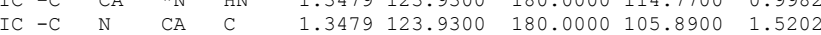

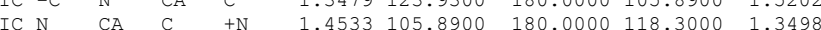

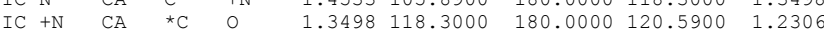

$\begin{array}{lllllllll}\text { IC } \mathrm{CA} \quad \mathrm{C} & +\mathrm{N} & +\mathrm{CA} & 1.5202 & 118.3000 & 180.0000 & 124.5000 & 1.4548\end{array}$

$\begin{array}{lllllllll}\text { IC } N & \mathrm{C} & { }^{*} \mathrm{CA} & \mathrm{CB} & 1.4533 & 105.8900 & 121.7900 & 111.9800 & 1.5584\end{array}$

$\begin{array}{lllllllll}\text { IC N } & \mathrm{C} & * \mathrm{*} \text { CA } & \mathrm{HAY} & 1.4533 & 105.8900 & -116.3400 & 107.7100 & 1.0837\end{array}$

$\begin{array}{lllllllll}\text { IC N } & \text { CA } & \text { CB } & \text { SG } & 1.4533 & 111.5600 & 180.0000 & 113.8700 & 1.8359\end{array}$

$\begin{array}{llllllrlrl}\text { IC } & \text { SG } & \text { CA } & { }^{*} \text { CB } & \text { HB1 } & 1.8359 & 113.8700 & 119.9100 & 107.2400 & 1.1134 \\ \text { IC } & \text { SG } & \text { CA } & { }^{*} \text { CB } & \text { HB2 } & 1.8359 & 113.8700 & -125.3200 & 109.8200 & 1.1124\end{array}$

IC CA CB SG FE $1.5408 \quad 113.0499 \quad 103.6324 \quad 105.5057 \quad 2.2544$ !from old file my top mod.inp

$\begin{array}{llllllll}\text { ! HEME } & & & & & \end{array}$

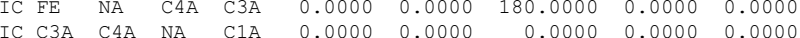

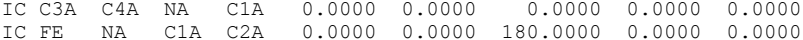

$\begin{array}{lllllrrrrr}\text { IC } & \text { FE } & \text { NA } & \text { C1A } & \text { C2A } & 0.0000 & 0.0000 & 180.0000 & 0.0000 & 0.0000 \\ \text { IC } & \text { C4A } & \text { NA } & \text { FE } & \text { NB } & 0.0000 & 0.0000 & 0.0000 & 0.0000 & 0.0000\end{array}$

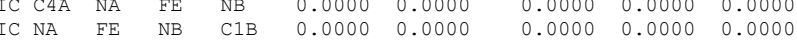

$\begin{array}{lllllllll}\text { IC } & \text { FE NB } & \text { C1B } & \text { C2B } & 0.0000 & 0.0000 & 180.0000 & 0.0000 & 0.0000\end{array}$

$\begin{array}{lllllllll}\text { IC } C 2 B & \text { C1B } & \text { NB } & \text { C4B } & 0.0000 & 0.0000 & 0.0000 & 0.0000 & 0.0000\end{array}$

$\begin{array}{llllllllll}\text { IC } & F E & \text { NB } & C 4 B & C 3 B & 0.0000 & 0.0000 & 180.0000 & 0.0000 & 0.0000\end{array}$

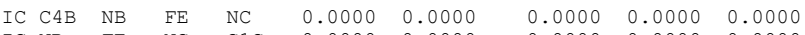

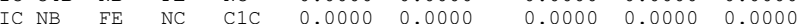

$\begin{array}{lllllllllllll} & \text { IC } & \mathrm{FE} & \mathrm{NC} & \mathrm{C} 1 \mathrm{C} & \mathrm{C} 2 \mathrm{C} & 0.0000 & 0.0000 & 180.0000 & 0.0000 & 0.0000\end{array}$

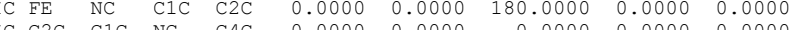

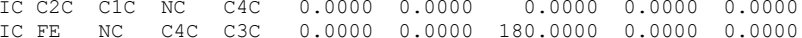

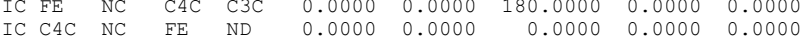

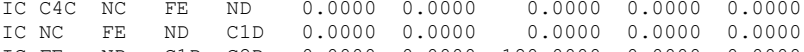

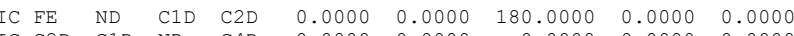

$\begin{array}{llllllll}\text { IC } & \text { C2D C1D ND C4D } & 0.0000 & 0.0000 & 0.0000 & 0.0000 & 0.0000\end{array}$

$\begin{array}{lllllllll}\text { IC } & \text { FE ND } & \text { C4D } & \text { C3D } & 0.0000 & 0.0000 & 180.0000 & 0.0000 & 0.0000\end{array}$

$\begin{array}{lllllllll}\text { IC C4A NA } & \text { FE } & \text { CHB } & 0.0000 & 0.0000 & 0.0000 & 0.0000 & 0.0000\end{array}$

$\begin{array}{lllllllll}\text { IC NA } & \text { FE } & \text { CHB } & \text { HB } & 0.0000 & 0.0000 & 0.0000 & 0.0000 & 0.0000\end{array}$

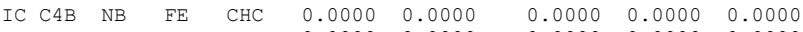

$\begin{array}{lllllllll}\text { IC NB } & \text { FE } & \text { CHC } & \text { HC } & 0.0000 & 0.0000 & 0.0000 & 0.0000 & 0.0000\end{array}$

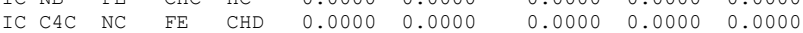

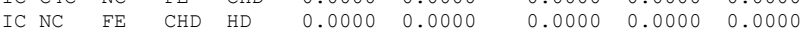

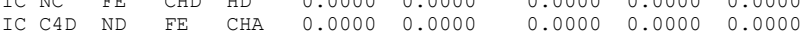

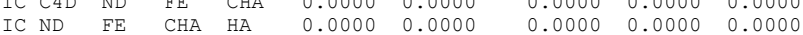

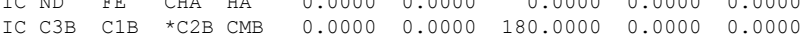

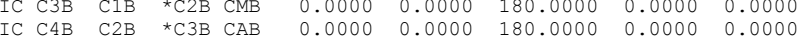

$\begin{array}{llllllllll}\text { IC } & \mathrm{C} 2 \mathrm{~B} & \mathrm{C} 3 \mathrm{~B} & \mathrm{CAB} & \mathrm{CBB} & 0.0000 & 0.0000 & -45.0000 & 0.0000 & 0.0000\end{array}$ ! PREVENTS VINYL COLLISION

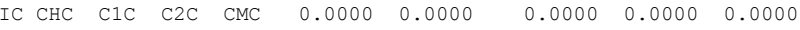

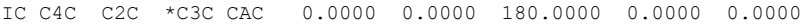

IC $C 2 C$ C3C CAC CBC $0.0000 \quad 0.0000 \quad-45.0000 \quad 0.0000 \quad 0.0000$ ! PREVENTS VINYL COLLISION

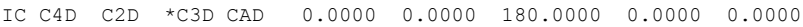

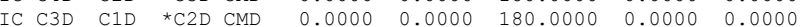

IC C2D C3D CAD CBD $0.0000 \quad 0.0000-120.0000 \quad 0.0000 \quad 0.0000$

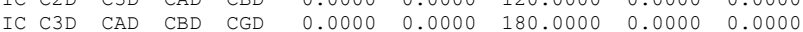

$\begin{array}{lllllll}\text { IC CAD CBD CGD O1D } & 0.0000 & 0.0000 & 0.0000 & 0.0000 & 0.0000\end{array}$

$\begin{array}{llllllll}\text { IC CAD CBD } & \text { CGD } & \text { O2D } & 0.0000 & 0.0000 & 180.0000 & 0.0000 & 0.0000\end{array}$

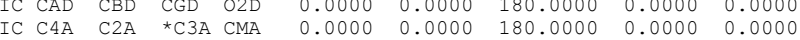

$\begin{array}{llllllllll}\text { IC } & \text { C3A } & \text { C1A } & { }^{*} \text { C } 2 \mathrm{~A} & \text { CAA } & 0.0000 & 0.0000 & 180.0000 & 0.0000 & 0.0000\end{array}$

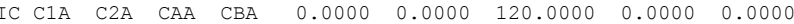

$\begin{array}{lllllllll}\text { IC C2A } & \text { CAA CBA } & \text { CGA } & 0.0000 & 0.0000 & 180.0000 & 0.0000 & 0.0000\end{array}$

$\begin{array}{lllllllll}\text { IC CAA } & \text { CBA } & \text { CGA } & 01 \mathrm{~A} & 0.0000 & 0.0000 & 0.0000 & 0.0000 & 0.0000\end{array}$

$\begin{array}{lllllllll}\text { IC CAA } & \text { CBA } & \text { CGA } & \text { O2A } & 0.0000 & 0.0000 & 180.0000 & 0.0000 & 0.0000\end{array}$

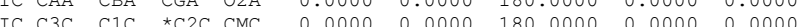

IC CBA $01 \mathrm{~A}$ *CAB O2A $\quad 0.0000 \quad 0.0000 \quad 180.0000 \quad 0.0000 \quad 0.0000$

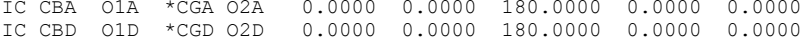

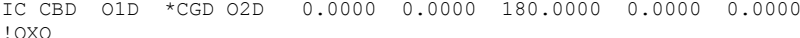

$\begin{array}{lllllllll}\text { IC CB } & \text { SG } & \text { FE } & \text { OF } 1 & 0.0000 & 0.0000 & 180.0000 & 0.0000 & 0.0000\end{array}$

PATCHING FIRS NONE LAST NONE

END 


\section{Heme- $\mathrm{HOOH}$}

271 !this is the version number

!Topology file for 1DZ9-- Conpound I of P450cam viewd by Schilchting et.al.

! Further modified to describe Fe-HOOH by Kyung-Bin Cho.

MASS 93 POT $39.10200 \mathrm{~K}$ ! Potassium Ion check masses

DECL $-C A$

DECL $-\mathrm{C}$

DECL $-O$

DECL +HN

$\mathrm{DECL}+\mathrm{CA}$

DEFA FIRS NTER LAST CTER

AUTO ANGLES DIHE

RESI POT $\quad 1.00$ ! Potassium Ion

ATOM POT РОT 1.00

PATCHING FIRS NONE LAST NONE

!naming the hem+cys toxo group with a total charge of -2.00

RESI HEH2 -1.00

GROUP

$\begin{array}{llrlr}\text { ATOM N } & \text { NH1 } & -0.47 & ! & \text { । } \\ \text { ATOM HN } & \text { H } & 0.31 & ! & \text { HN-N }\end{array}$

АTOM CA CT1 $0.07 \quad$ !

$0.07 ! \quad$ I

HAY-CA--CB--(SG) move definition of HA-->HAY

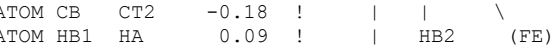

ATOM HB2 HA $0.09 ! \quad \mathrm{O}=\mathrm{C}$

GROUP

$\begin{array}{lll}\text { ATOM C } & \text { C } & 0.5\end{array}$

АTOM $0 \quad 0 \quad-0.51$

ladding the heme

GROUP

ATOM SG S 0.23 ! the charge on the HGI atom included in the SG GROUP

ATOM OF1 O $\quad-0.22$ ! the oxo group

ATOM HF1 H 0.26

ATOM OF2 O -0.22

ATOM HF2 H 0.26 !

ATOM FE FE 0.53 !

ATOM NA NPH -0.38 !

ATOM NB NPH -0.38

ATOM NC NPH -0.38

$\begin{array}{lll}\text { ATOM ND NPH } & -0.38 \\ \text { ATOM } & \text { N1A } & \text { CPA }\end{array}$

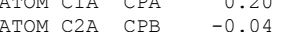

ATOM C3A CPB -0.04 !

ATOM C4A CPA $\quad 0.20 !$

ATOM $\mathrm{C} 2 \mathrm{~B} \quad \mathrm{CPB}$

ATOM C3B CPB

АTOM C4B CPA

$\begin{array}{lll}\text { ATOM } & \text { C1C } & \text { CPA } \\ \text { ATOM } & \text { C2C } & \text { CPB }\end{array}$

ATOM C2C CPB

ATOM C3C CPB

ATOM C1D CPA

ATOM C2D CPB

ATOM C3D CPB

ATOM C4D CPA

GROUP

ATOM CHA CPM

ATOM HA HA

GROUP

ATOM CHB CPM

ATOM HB HA

GROUP

ATOM HC HA $\quad-0.05$ !

GROUP

-0.04 ! HMA $\backslash$ । 11 ।

0.20 ! HMA3/ $/ / \backslash 1 / /, / /$ IHMD3

0.20

-0.04 !

$-0.04$

0.20

$-0.04 !$ HMB 1

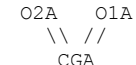

$\backslash \backslash / /$

HBA1--CBA--HBA2 HA HBD1--CBD--HBD2

HAA1--CAA

$\mathrm{C} 2 \mathrm{~A}---\mathrm{C} 1 \mathrm{~A}-\mathrm{C} 4 \mathrm{D}---\mathrm{C} 3 \mathrm{D}$

I $1 / / 1$

$0.20 !$ HMB $3 /$

$-0.05 !$

0.15

$-0.05 !$

0.15

$0.15 !$

ATOM CHD CPM $\quad-0.05$

ATOM HD HA 0.15

GROUP

ATOM CMA CT3 $\quad-0.27$

$\begin{array}{ll}\text { ATOM HMA } 1 \mathrm{HA} & 0.09\end{array}$

ATOM HMA2 HA 0.09

ATOM HMA 3 HA

GROUP

ATOM CAA CT2 -0.18

ATOM HAA1 HA 0.09

ATOM HAA2 HA

GROUP

ATOM CBA CT2 -0.28

ATOM HBA1 HA 0.09

ATOM HBA2 HA 0.09

ATOM CGA CC $\quad 0.62$

ATOM O1A OC $\quad-0.76$

ATOM O2A OC $\quad-0.76$

GROUP 


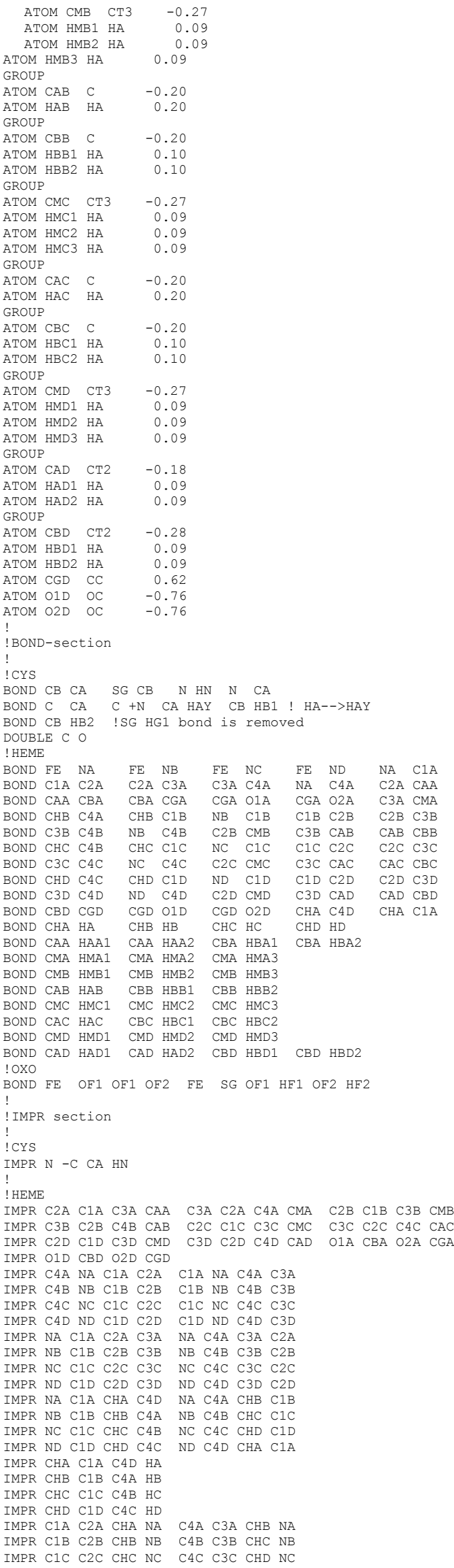




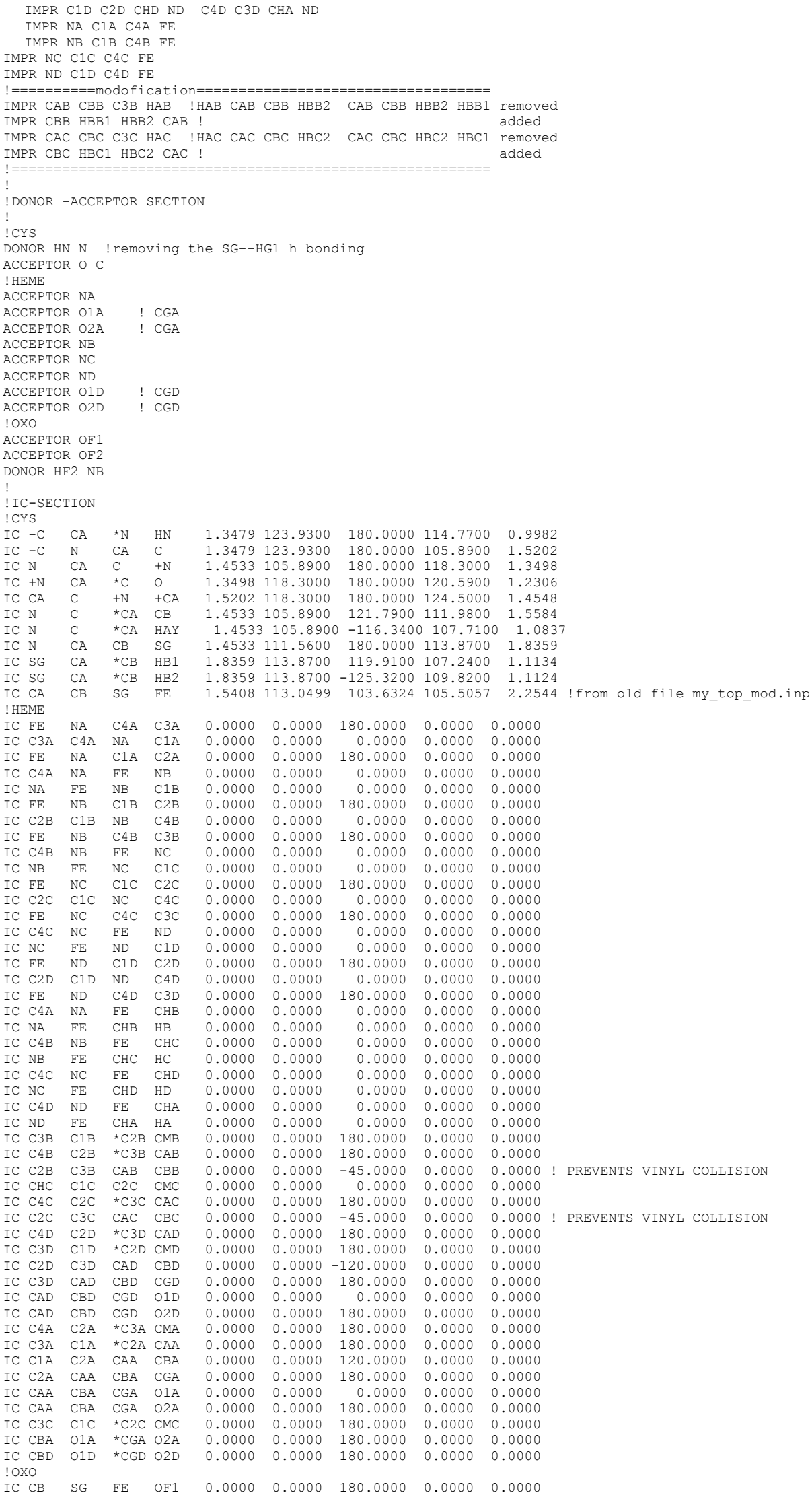

